\title{
An inverse approach to understanding benthic oxygen isotope records from the last deglaciation
}

by

\begin{abstract}
Daniel Edward Amrhein
B.A., Columbia University (2009)

Submitted in partial fulfillment of the requirements for the degree of Master of Science

at the

MASSACHUSETTS INSTITUTE OF TECHNOLOGY

and the

WOODS HOLE OCEANOGRAPHIC INSTITUTION

February 2014

(C) 2014 Daniel Edward Amrhein. All rights reserved.

The author hereby grants to MIT and WHOI permission to reproduce and to distribute publicly paper and electronic copies of this thesis document in whole or in part in any medium now known or hereafter created.
\end{abstract}

Signature of author

Joint Program in Oceanography - Massachusetts Institute of Technology/

Woods Hole Oceanographic Institution

22 November 2013

Certified by

Carl Wunsch

Cecil and Ida Green Professor of Physical Oceanography, Emeritus

Massachusetts Institute of Technology

Thesis Advisor

Accepted by

Glenn Flierl

Professor of Physical Oceanography

Massachusetts Institute of Technology

Chair, Joint Committee on Physical Oceanography 


\title{
An inverse approach to understanding benthic oxygen isotope records from the last deglaciation
}

\author{
by \\ Daniel Edward Amrhein \\ Submitted to the \\ Massachusetts Institute of Technology / Woods Hole Oceanographic Institution \\ Joint Program in Oceanography \\ on November 22, 2014 \\ in partial fulfillment of the requirements for the degree of \\ Master of Science
}

\begin{abstract}
Observations suggest that during the last deglaciation (roughly 20,000-10,000 years ago) the Earth warmed substantially, global sea level rose approximately 100 meters in response to melting ice sheets and glaciers, and atmospheric concentrations of carbon dioxide increased. This interval may provide an analog for the evolution of future climate. The ocean plays a key role in the modern climate system by storing and transporting heat, salt, and nutrients, but its role during the last deglaciation remains uncertain.

Prominent signals of the last deglaciation in the ocean are a gradual warming and a decrease of the seawater oxygen isotope ratio $\delta^{18} \mathrm{O}$ (a signature of melting land ice sheets). These changes do not occur uniformly in the ocean, but propagate like plumes of dye over hundreds and thousands of years, the aggregate results of turbulent advective and diffusive processes. Information about changing temperatures and oxygen isotopes is stored in the shells of benthic organisms recovered in ocean sediment cores.

This thesis develops and applies an inverse framework for understanding deglacial oxygen isotope records derived from sediment cores in terms of the Green functions of ocean tracer transport and ocean mixed layer boundary conditions. Singular value decomposition is used to find a solution for global mixed layer tracer concentration histories that is constrained by eight last-deglacial sediment core records and a model of the modern ocean tracer transport. The solution reflects the resolving power of the data, which is highest at model surface locations associated with large rates of volume flux into the deep ocean. The limited data resolution is quantified and rationalized through analyses of simple models.

The destruction of information contained in tracers is a generic feature of advectivediffusive systems. Quantifying limitations of tracer records is important for making and understanding inferences about the long-term evolution of the ocean.
\end{abstract}

Thesis Supervisor: Carl Wunsch

Title: Cecil and Ida Green Professor of Physical Oceanography, Emeritus 


\section{Contents}

1 Introduction 13

1.1 Roles for the ocean in the last deglaciation . . . . . . . . . . . 14

1.2 Previous applications of ocean tracers in deglacial paleoclimatology, with an emphasis on $\delta^{18} \mathrm{O} \ldots \ldots \ldots \ldots$

2 Tracer inverse problems in steady flows $\quad 27$

2.1 Notation . . . . . . . . . . . . . . . . . . . . . . . . . . 28

2.2 Machinery: Least Squares and SVD . . . . . . . . . . . . . . 30

2.3 Forward and inverse tracer problems . . . . . . . . . . 36

2.4 Inference from tracers in a four-box model . . . . . . . . . . 50

2.5 Inference of steady flow: Models of Gebbie and Huybers [2010, 2012] 66

3 Benthic records of $\delta^{18} \mathrm{O}_{c c} \quad 71$

3.1 Records of $\delta^{18} \mathrm{O}_{c c} \ldots \ldots \ldots \ldots \ldots \ldots \ldots \ldots \ldots \ldots \ldots$

3.2 Descriptions of sediment core records . . . . . . . . . . . . 74

3.3 Error models, mean estimation, and objective mapping . . . . . . . 82

4 The deglacial benthic $\delta^{18} \mathrm{O}$ tracer problem $\quad 91$

4.1 Assumptions . . . . . . . . . . . . . . . . . . . . 92

4.2 Formulation of the inverse boundary problem . . . . . . . . 95

4.3 Results . . . . . . . . . . . . . . . . . . . . . . 105

4.4 Further discussion of the $K^{\prime}=400$ case $\ldots \ldots \ldots \ldots \ldots$

4.5 Discussion . . . . . . . . . . . . . . . . . . . . . . . . . . 129

5 Concluding remarks 133 


\section{List of Figures}

1.1 Chronologies of deglacial proxies for surface climate over the interval 25-10 kya. Colored bars indicate approximate ranges for millennial "events": purple is Heinrich Event 1 (H1); light blue is the Bolling Allerod (BA); mauve is the Younger Dryas (YD). TOP: Cariaco Basin SST record derived from planktonic foraminiferal measurements of $\mathrm{Mg} / \mathrm{Ca}$ [Lea et al., 2003]. MIDDLE: $\delta^{18} \mathrm{O}_{i c e}$ in permil deviations from VSMOW for the GISP2 Greenland ice core record (blue, Grootes et al. [1993], Stuiver et al. [1995]) and the EPICA Dome C ice core record (green, Stenni et al. [2001]). BOTTOM: Sea level measured at Barbados using corals, reported relative to the modern and corrected for isostatic rebound $[$ Peltier and Fairbanks, 2006]. . . . . . . . . . . .

2.1 A box model with four reservoirs related by constant volume flux rates. The subscript $\infty$ denotes that the flow is out of the system. . . . .

2.2 The discrete-time impulse response $\zeta_{i j}(t)$ describes the evolution of tracer concentration at the $i^{\text {th }}$ state element in response to an "impulse" in the $j^{\text {th }}$ control element. Panel (a) shows the impulse, which is 0 at all times except $t=0$, when it is 1 . Panel (b) shows the impulse response in each of the two state boxes due to impulses in the two control boxes. $\zeta_{41}(t)$ and $\zeta_{42}(t)$ are the same. Measurable tracer concentrations in the state boxes are smoothed and phase-shifted relative to the impulse. The transfer function is the Fourier transform of the impulse response; the real and imaginary parts of the transfer function are plotted in (c) and (d) and describe the attenuation and lag of the initial impulse experienced in each state box as a function of frequency, respectively. 
2.3 Top: Singular values for the time-dependent four-box tracer inverse boundary problem decay rapidly with SVD index from a few high values to values roughly an order of magnitude smaller. Bottom: Left and right singular vector pairs corresponding to SVD indices 1, 2, 19, and 20 plotted as time series for each box. Left singular vectors show structures in the observable boxes 3 and 4 , while right singular vectors $v_{i}$ describe structures in the control boxes 1 and 2. The offset in time axes results from the one time-step delay in control inference. The lower amplitude of Box 3 relative to Box 4 in the right singular vectors is the result of the smaller flux into the observable boxes from Box 3 .

3.1 Locations for the eight sediment core records of $\delta^{18} \mathrm{O}$ used in this study. Labels are abbreviations based on ocean basin and water depth; the original core names are found in Table (3.1). . . . . . . . . . .

3.2 The eight benthic foraminiferal $\delta^{18} \mathrm{O}$ records used in this work plotted about their means. Computation of record mean values is discussed in section 3.3.2. Grid spacing in the vertical is $1 \%$ and in the horizontal is 1000 years. Some records contain measurements from multiple benthic foraminferal species. . . . . . . . . . . . . .

3.3 Similar to Figure 3.2, but including objectively-mapped samples (black lines) and their uncertainty (shading) every 200 years. Grid spacing in the vertical is $1 \%$ and in the horizontal is 1000 years. The plotted uncertainties are the square roots of the diagonal of the uncertainty $\operatorname{matrix} \mathbf{P}($ Eqn 3.6). . . . . . . . . . . . . . . . . . . .

4.1 Seven surface regions defined in Gebbie and Huybers [2010]. Heaviside responses in Figures 4.2 and 4.3 were generated by enforcing uniform concentrations of 1 in the mixed layer of the North Atlantic (NATL, cyan), and the Antarctic (ANT, blue) regions. . . . . . . . . . . 100

4.2 Heaviside response in the TMI steady circulation estimate when the mixed layer in the North Atlantic (see the definition of this region in Figure 4.1) is held at a fixed concentration of 1 beginning at $t=0$. Each column shows snapshots of the system state at three times (100, 500, and 4000 years) for a given depth (1000, 2000, and 3000 meters).

4.3 Same as Figure 4.2, but for a Heaviside tracer forcing in a band of the Southern Ocean (see the definition of this region in Figure 4.1). . . . 102

4.4 The solution to the initial value problem. Panel 4.4a shows the singular values of the initial value problem and panel $4.4 \mathrm{~b}$ plots the solution $\tilde{\mathbf{q}}_{0}$. A single maximum in the Weddell Sea accounts for most of the variance, but there are nonzero values distributed throughout the North Atlantic, Arctic, and Southern Oceans. Units of both plots are $\% 0 \delta^{18} \mathrm{O} . \quad \ldots .107$ 
4.5 SVD solution range vectors are weighted by $\frac{\mathbf{u}_{i}^{\top} \overline{\mathbf{f}}}{\lambda_{i}}$ (Equation (4.2)) and thus reflect both the singular values $\lambda_{i}$ (panel $4.5 \mathrm{a}$ ) and the projection $\mathbf{u}_{i}^{\top} \overline{\mathbf{f}}$ of the mean-removed records onto the left singular values of the weighted matrix $\left[\overline{\mathbf{W}}^{-\top / 2} \overline{\mathbf{B}} \overline{\mathbf{G}} \overline{\mathbf{F}}\right]$. The number of singular values is 785 , corresponding to the minimum dimension of $\left[\overline{\mathbf{W}}^{-\top / 2} \overline{\mathbf{B}} \overline{\mathbf{G}} \overline{\mathbf{F}}\right]$. . . . . .

4.6 The solution $\tilde{\mathbf{q}}$ obtained with effective nullspace parameter $K^{\prime}=300$ for the time evolution of "fluctuation" (mean-removed) surface $\delta^{18} \mathrm{O}_{c c}$ is plotted as three EOF-PC pairs. EOF-PC pairs are computed by finding the singular value decomposition of the whole-domain solution vector, reordered into a matrix indexed by space in rows and time in columns (see Section ). The EOFs (spatial patterns, right panels) are shown in normalized units, while the PCs (time series, left panels) have the units of the tracer. Times and regions of zero value lie in the solution null space of the problem. The solution has low values everywhere at the end of the reconstruction interval because of lags in transporting tracer to observable regions. . . . . . . . . . . . . . . 114

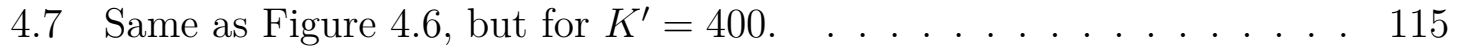

4.8 Raw data (blue dots), objectively mapped data (black lines), 1- $\sigma$ uncertainty (shaded regions), and reconstructed data $\tilde{\overline{\mathbf{f}}}$ plotted by sediment core for the choice of nullspace parameter $K^{\prime}=300$. All solutions are for the fluctuating part of the problem, and each raw dataset has had the least-squares estimate $\tilde{m}$ of its mean removed. . . . . . . . . . . 117

4.9 Same as 4.8, but for $K^{\prime}=400$. . . . . . . . . . . . . . . . . . . 118

4.10 The diagonal elements of the solution covariance matrix $\mathbf{C}_{\tilde{\tilde{\mathbf{q}}} \tilde{\mathbf{q}}}$ (the "standard error") constitute a spatiotemporal pattern with the same dimension as $\tilde{\overline{\mathbf{q}}}$. Here, the standard error is plotted in terms of its leading EOF-PC pairs for the $K^{\prime}=300$ (4.10a) and $K^{\prime}=400$ (4.10b) cases. The EOFs (spatial patterns, right panels) are shown in normalized units, while the PCs (time series, left panels) have the units of the tracer. Times and regions of zero concentration lie in the solution null space of the problem. The leading EOF-PC pair accounts for over $97 \%$ of the variance in both cases and is a good representation of the standard error. . . . . . . . . . . . . . . . . .

4.11 Three consecutive columns of the solution resolution matrix $\mathbf{T}_{v}$ show sidelobes in time and space in the inference of a delta function of tracer at the location marked by a green circle (top left) at $t=-15000$. Note that color maps between panels differ by factors of 10. Rather than reconstructing the delta function, the network of eight sediment cores infers a pattern of nonzero boundary conditions smeared out over the North Atlantic and Arctic Oceans, with a small amount inferred as originating 200 years earlier. . . . . . . . . . . . . . . 
4.12 Diagonal elements of $\mathbf{T}_{v}$ selected at $t=-15000$ show how variance is attenuated in estimates of surface boundary conditions $\overline{\mathbf{q}}$. For instance, a delta function of tracer released at $t=-15000$ in the Weddell Sea (lower right hand corner, red pixel) will be reconstructed at $t=-15000$ with a value of 0.94 (with additional variance in spatial and temporal sidelobes). For the great majority of surface points, very little or no variance is reconstructed faithfully. . . . . . . . . . . .

4.13 Objectively mapped data (black lines), 1- $\sigma$ uncertainty (shaded regions), reconstructed data $\tilde{\overline{\mathbf{f}}}$ (red), and "data importance" (green) plotted for each core. The data importance is the diagonal of the data resolution matrix $\mathbf{T}_{u}$ and describes the contribution of the $i^{\text {th }}$ element of $\overline{\mathbf{f}}$ to the $i^{t h}$ element of the reconstructed data $\tilde{\overline{\mathbf{f}}}$. . . . . . . . . . . 125

4.14 Histogram of the scaled residuals $\overline{\mathbf{W}}^{-\mathrm{T} / 2} \tilde{\overline{\mathbf{n}}}$. The distribution is nonGaussian at the $5 \%$ confidence level. . . . . . . . . . . . .

4.15 TOP: Modern climatological values of salinity in the Atlantic Ocean at $26^{\circ}$ north latitude [Gouretski and Koltermann, 2004]. BOTTOM: Values of $\delta^{18} \mathrm{O}_{c c}$ anomaly at the same location in a forward integration of the GH12 tracer transport model forced by controls derived in the inverse problem with $K^{\prime}=400 . \quad$. . . . . . . . . . . . . . . . . . . . 128

4.16 The global ocean volume is filled disproportionately in the steady pathways model of Gebbie and Huybers [2010] by a subset of surface grid boxes. In this figure, circle radii are propotional to the proportion of ocean volume below the mixed layer that originates from the corresponding surface locations. Figure 2 from Gebbie and Huybers [2011] is a logarithmic version of this plot. . . . . . . . . . . . 


\section{List of Tables}

3.1 Summary of benthic foraminferal $\delta^{18} \mathrm{O}$ records. The "Name" field indicates reference codes used in this thesis for ease of discussion. SA1967 refers to a South Atlantic core at $1967 \mathrm{~m}$ depth, NI1580 to a North Indian core at $1580 \mathrm{~m}$ depth, etc. . . . . . . . . . . . . . . . . .

4.1 Properties of the data as used in the inverse model. Locations of sediment cores were interpolated to TMI ocean grid box locations by minimizing differences in depth, longitude, and latitude. . . . . . . . 105

4.2 Data, reconstructed data, and residuals for the initial value problem. 107 


\section{Chapter 1}

\section{Introduction}

Our understanding of the physics of the ocean and climate is limited by the length of the instrumental period. Direct measurements of the climate system, whether from satellites, hydrographic sections, drifter pathways, or anything else, provide what is effectively a snapshot of a system that is believed to vary on all time scales. Moreover, while the climate response to large-amplitude, high-frequency, external forcings such as the diurnal or annual cycles can be quite profound, we have not observed how the climate responds to forcings of similar magnitudes on longer time scales. Inference of the evolution of past climates from paleoclimate archives can aid our understanding of the physics underlying low-frequency variability in the climate system and may provide analogs for future climate change.

This work focuses on interactions between the ocean and surface climate during the interval from 25 kya (25000 years before present) to 5 kya. During this time Earth's climate underwent major changes as it evolved from the Last Glacial Maximum (the period of maximum ice volume during the last glacial interval, roughly $20 \mathrm{kya}$ ) to the warmer Holocene climate (roughly the last $10 \mathrm{kya}$ ). During this time, sea level 
rose by roughly 120 meters as meltwater from land ice entered the ocean and the air warmed by as much as 10 degrees in high latitudes.

The first chapter of this thesis provides background on the last deglaciation and the role of the ocean therein. Some of the literature on the study of ocean tracers during the last deglaciation is reviewed, with an emphasis on the use of the oxygen isotope ratio as expressed by the measured quantity $\delta^{18} \mathrm{O}$. Chapter 2 describes a framework for the inference of tracer boundary conditions in steady flow from tracer observations in the system interior and provides examples using a simple four-box model. Chapter 3 describes eight sediment core records of $\delta^{18} \mathrm{O}_{c c}\left(\delta^{18} \mathrm{O}\right.$ measured in calcium carbonate derived from benthic foraminifera) and an objective mapping to construct evenly-sampled records and uncertainty estimates. Chapter 4 applies the inverse methods developed in Chapter 2 to the eight sediment cores to estimate ocean mixed-layer $\delta^{18} \mathrm{O}_{c c}$. Finally, Chapter 5 discusses the results, assesses the utility and shortcomings of the techniques developed, and suggests future directions for research.

\subsection{Roles for the ocean in the last deglaciation}

Several records of deglacial surface climate are plotted in Figure 1.1. The panel first is a time series of sea surface temperature (SST) in the Cariaco basin derived from planktonic foraminifera. The second shows time series of the oxygen isotope ratios of ice, $\delta^{18} \mathrm{O}_{i c e}$, from Greenland (GISP) and Antarctica (EPICA Dome C), which are linearly related to surface air temperature (SAT) at the ice core locations. The third plot shows changes in sea level relative to the modern as derived using Barbados corals [Peltier and Fairbanks, 2006]. These time series summarize the major surface climate shifts over 20-10 kya: Earth became warmer, especially at the poles, and sea level rose by roughly $80 \mathrm{~m}$. The overall deglacial transitions evident in Figure 1.1 

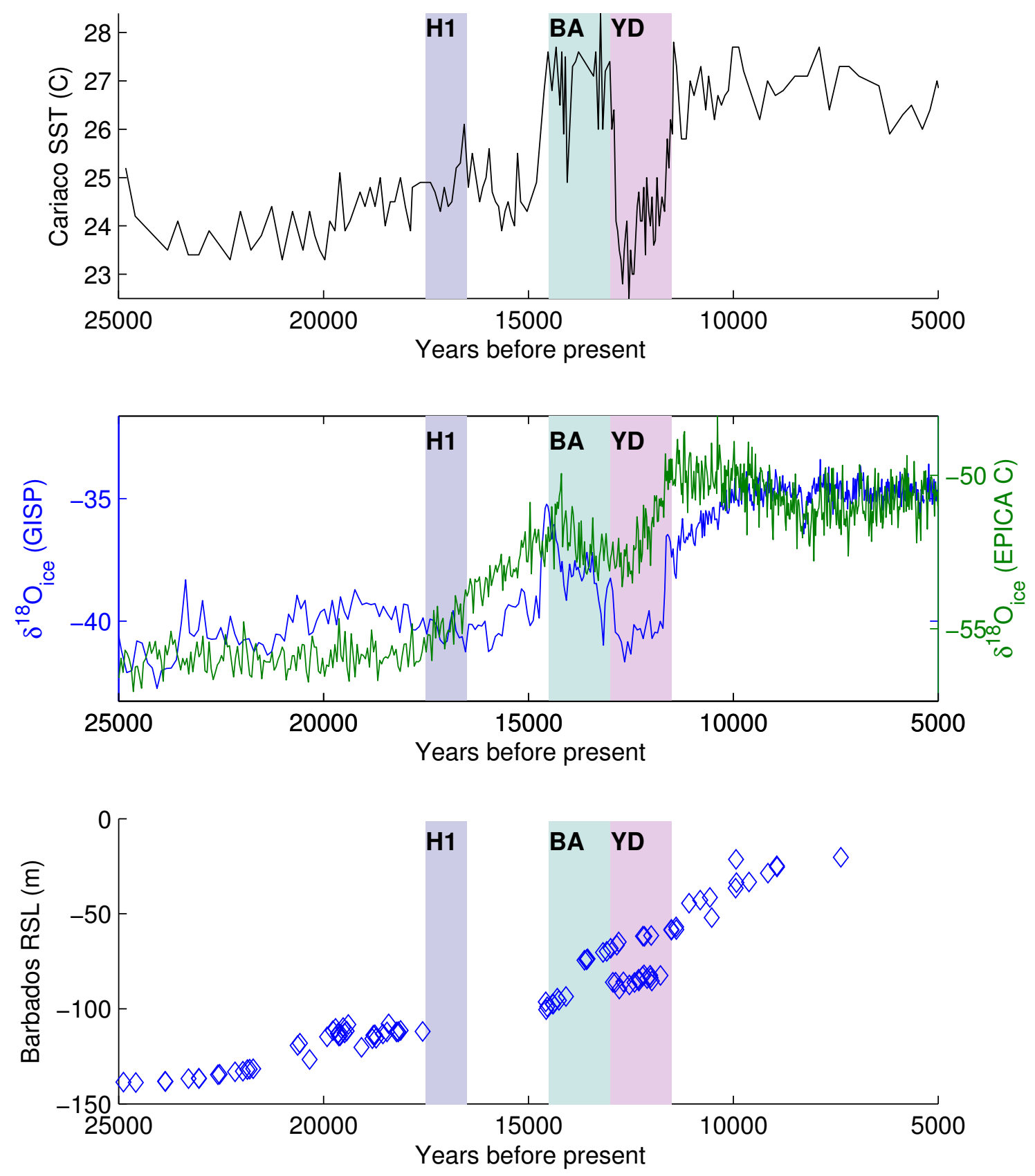

Figure 1.1: Chronologies of deglacial proxies for surface climate over the interval 2510 kya. Colored bars indicate approximate ranges for millennial "events": purple is Heinrich Event 1 (H1); light blue is the Bolling Allerod (BA); mauve is the Younger Dryas (YD). TOP: Cariaco Basin SST record derived from planktonic foraminiferal measurements of $\mathrm{Mg} / \mathrm{Ca}$ [Lea et al., 2003]. MIDDLE: $\delta^{18} \mathrm{O}_{\text {ice }}$ in permil deviations from VSMOW for the GISP2 Greenland ice core record (blue, Grootes et al. [1993], Stuiver et al. [1995]) and the EPICA Dome C ice core record (green, Stenni et al. [2001]). BOTTOM: Sea level measured at Barbados using corals, reported relative to the modern and corrected for isostatic rebound [Peltier and Fairbanks, 2006]. 
are part of a glacial-interglacial cycle that is a dominant signal in records of climate over the past million years and is believed to be coupled to changes in Earth's orbit around the sun [Hays et al., 1976, Huybers and Wunsch, 2005] and greenhouse gas concentrations [Shakun et al., 2012].

A large paleoceanographic and geochemical literature is dedicated to studying differences between the LGM and modern ocean states - the initial and final conditions of the deglaciation [Curry et al., 1988, Curry and Oppo, 2005, Wunsch, 2003, Lynch-Stieglitz et al., 1999, Marchal and Curry, 2008, Dail, 2012, Lund et al., 2011, and many others]. Curry and Oppo [2005] obtained benthic foraminiferal oxygen and carbon isotopes from sediment cores recovered at a range of depths on the Brazil Margin. They concluded that, within the western Atlantic, deep ocean waters formed in the North Atlantic fed a more intermediate water mass than in the modern ocean and that deep water from the Southern Ocean filled more of the basin. Keigwin and Schlegel [2002] argued that ventilation ages (the time since a parcel was exposed to the atmosphere, as measured by ${ }^{14} \mathrm{C}$ ages) during several time slices within the LGM (21-18 kya) were roughly five times as great as those found in modern NADW. Marchal and Curry [2008] used an inverse method to show that about 20\% of LGM estimates of $\delta^{18} \mathrm{O}_{c c}$ and $\delta^{13} \mathrm{C}$ in the Atlantic are inconsistent with modern estimates, but only if certain assumptions about sediment core measurement error and tracer transport are made. Many estimates of Holocene and LGM ocean properties assume that the ocean was in a steady state over a long interval, which can introduce substantial errors (see Section 3.3.2). Conjectures of differences between ocean states at the LGM and in the Holocene provide testable hypotheses for the long-term evolution of the abyssal circulation.

In addition to the overall glacial-interglacial change, features in some deglacial records have been interpreted as "events" that may have global or regional extent. 
In particular, three shifts in geochemical records persisting for roughly 1000 years "Heinrich Event 1" (H1), ca. 17.5-16.5 kya, the "Bolling/Allerod" (BA), ca. 14.513 kya, and the "Younger Dryas" (YD) or "Heinrich Event 0", ca. 13-11.5 kya are probably the best-studied features of the last deglaciation. Qualitatively similar structures have been observed in preceding glacial and interglacial intervals, where they are sometimes lumped together as "millennial variability." It is unclear whether the $\mathrm{YD}, \mathrm{BA}$, and $\mathrm{H} 1$ are modes of climate variability that are superimposed upon more gradual deglacial changes, or whether millennial variability played an intrinsic role in the glacial-interglacial transition.

Alley et al. [2003] and Broecker [2003] suggested that features like the YD and BA could be explained by a local climate instability that is amplified by feedbacks and communicated (via a "globalizer") across different hemispheres. The perturbed climate state must be maintained for hundreds or thousands of years, requiring a so-called "flywheel." The "globalizer" and "flywheel" concepts are frequently used to implicate the ocean because the ocean can transport properties over large distances and has long tracer equilibration time scales.

On time scales shorter than that of the deglaciation, many studies have suggested that the meridional mass transport of the Atlantic Ocean (the Atlantic Meridional Overturning Circulation or AMOC) changes as a response to freshwater input at high latitudes [Keigwin et al. [1991], Keigwin and Jones [1994], Alley et al. [2003], Broecker [2003]]. In this paradigm, freshwater released in the high latitudes of the North Atlantic forms a low-density "lid" that stabilizes the water column to deep convection and reduce the creation of the modern North Atlantic Deep Water (NADW).

Numerous studies (so-called "hosing" experiments) have investigated the sensitivity of the high-latitude North Atlantic to freshwater forcing in coupled oceanatmosphere GCMs and have found reduced NADW formation and multiple regimes 
of flow [Manabe and Stouffer, 1988]. Additional modeling studies have concluded that the AMOC has multiple stable equilibria and exhibits hysteresis behavior, such that freshwater forcing can trigger rapid transitions between different AMOC regimes but recovery times to the initial state can take hundreds or thousands of years [Rahmstorf, 1995]. However, Condron and Winsor [2011] suggest that better-resolved GCMs distribute meltwater along western boundaries rather than exporting it to the open ocean where deep convection occurs. Eisenman et al. [2009] suggested that reductions in deepwater formation could have been caused by rain driven by receding ice sheets. It is difficult to assess the likelihood of such scenarios given that the unforced variability of the AMOC is poorly constrained (and even what is meant by the AMOC must be carefully specified; see Wunsch and Heimbach [2013]).

AMOC changes have also been cited to explain the apparent interhemispheric lags in temperature anomalies between Greenland and Antarctic ice cores during so-called Dansgaard-Oeschger events [Dansgaard et al., 1993, Blunier and Brook, 2001]. The "bipolar seesaw hypothesis" posits that, subsequent to AMOC reductions due to melting from regional warming, the deep Atlantic ocean is filled with water from the Southern Hemisphere, and increased deep-water formation in the Southern Ocean results in a local increase in surface air temperature [Broecker, 1998]. In this instance, the interhemispheric heat transport of the meridional ocean circulation serves to "globalize" the regional temperature perturbation.

The ocean is also thought to play an important role in melting land ice through the interaction with ice-sheet margins, particularly during times of sea level change. The barotropic response of the ocean to mass fluxes is extremely rapid and can be viewed as another potential "globalizer," allowing local ice sheet dynamics to have an indirect effect on ice margins elsewhere. However, the role of rising global sea level in melting ice-sheet margins and changing land ice flow rates is poorly constrained 
and very complicated. The intuitive notion that submerging a tongue of ice may destabilize it is complicated by changes in local gravity [Gomez et al., 2010] and compensating effects of sedimentation on grounding line location [Alley et al., 2007].

Hypothetical changes in ocean temperature or circulation could also have played a role in the melting of land ice by bringing ice into contact with warmer water. Pronounced differences in melt rates are observed on modern Antarctic ice shelves depending on the temperature of underlying waters [Joughin et al., 2012], and warminginduced thinning is believed to have been responsible for the recent loss of the Jakobshavn Isbrae floating ice tongue in Greenland [Joughin et al., 2008]. However, it is unclear to what extent one must implicate large-scale changes in ocean heat content or circulation in order to induce melting.

In summary, numerous hypotheses have been advanced regarding the role of the ocean in deglacial climate change. Constraining the evolution of the ocean's mass circulation during the deglacial interval is an important step in understanding the evolution of climate on millennial and longer time scales and the response of the ocean to low-frequency forcing.

\subsection{Previous applications of ocean tracers in deglacial paleoclimatology, with an emphasis on $\delta^{18} \mathrm{O}$}

The use of tracers in the ocean to infer flow properties is one of the oldest and most useful procedures in oceanography. Using the so-called dynamic method, modern shipboard observers gather measurements of temperature and salinity to construct multiple vertical density profiles, which are then incorporated in the thermal wind equation to yield vertical shear. An interior flow condition, typically a "level of no 
motion," can then be used to determine absolute velocities and thus to estimate the transport.

Unfortunately, information about vertical density profiles in paleoclimates is scarce and difficult to interpret [Lynch-Stieglitz et al., 1999, Wunsch, 2003, Huybers and Wunsch, 2010]. Nevertheless, most of what is known about past ocean circulation states has been derived from records of tracer concentrations, albeit recording properties other than density, that can be retrieved from foraminifera, bulk sediment, or pore waters deposited beneath the seafloor. Commonly used paleoproxy tracer quantities include the isotope ratios $\delta^{18} \mathrm{O}, \delta^{13} \mathrm{C}$, and $\Delta^{14} \mathrm{C}$, and the elemental ratios $\mathrm{Mg} / \mathrm{Ca}, \mathrm{Sm} / \mathrm{Nd}, \mathrm{Cd} / \mathrm{Ca}$, and ${ }^{231} \mathrm{~Pa} /{ }^{230} \mathrm{Th}$.

In the absence of dynamical constraints, a "clock" is necessary to constrain flow rates (as opposed to flow paths) [LeGrand and Wunsch, 1995, Huybers et al., 2007]. A so-called "age tracer" that sets a relative age condition for a water parcel can yield information about flow rates. For example, surface ocean concentrations of radioactive ${ }^{14} \mathrm{C}$ tend to equilibrate with atmospheric values through air-sea gas exchange, while parcels isolated from the atmosphere decay radioactively. Determining flow patterns and rates from such tracers, e.g. ${ }^{14} \mathrm{C} /{ }^{12} \mathrm{C}$ ratios, is an analytically challenging problem, with numerous time scales of importance [Wunsch, 2002].

Another geochemical measurement used to infer mass flux rates is the isotopic ratio ${ }^{213} \mathrm{~Pa} /{ }^{230} \mathrm{Th}$. These isotopes are differentially particle reactive in the water column and their ratio has been interpreted as a local proxy for column-averaged advection. McManus et al. [2004] measured ratios of ${ }^{213} \mathrm{~Pa} /{ }^{230} \mathrm{Th}$ excess in sediments at Bermuda Rise which they related to the strength of the AMOC. The authors postulated a slightly reduced AMOC during the LGM and the YD and a complete halt in overturning during H1. Subsequent work has documented issues with these interpretations, including varying particulate fluxes [Lippold et al., 2011], biases from opal 
fluxes [Bradtmiller et al., 2007], and questions about the vertical extent of velocity averaging [Thomas et al., 2006]. Burke et al. [2011] used an inverse method to find that a compilation of ${ }^{213} \mathrm{~Pa} /{ }^{230} \mathrm{Th}$ measurements from the Holocene, LGM, and $\mathrm{H} 1$ did not provide evidence of differences in circulation between those intervals when uncertainties in the data and their model were taken into account. Nevertheless, ${ }^{213} \mathrm{~Pa} /$ ${ }^{230} \mathrm{Th}$ is frequently cited alongside hosing studies in support of reductions in deepwater formation and/or AMOC transport during stadials. A sluggish stadial AMOC is an a priori assumption in many tracer studies of the LGM or Heinrich events.

The oxygen isotope ratio $\delta^{18} \mathrm{O}$ is described in permil deviation from a standard value,

$$
\delta^{18} \mathrm{O} \equiv\left[\frac{\left(\mathrm{O}^{18} / \mathrm{O}^{16}\right)_{\text {sample }}}{\left(\mathrm{O}^{18} / \mathrm{O}^{16}\right)_{\text {standard }}}-1\right] * 1000 \%
$$

where the "standard" is typically Pee Dee Belemnite (PDB) for carbonates and Vienna Standard Mean Ocean Water (VSMOW) for water and ice. Differences in the $\delta^{18} \mathrm{O}$ values of various substances arise because of mass-dependent fractionation. Compounds containing the lighter of the three stable isotopes of oxygen, ${ }^{16} \mathrm{O}$, have less mass and thus are more likely to evaporate and less likely to condense and rain out of clouds than compounds containing heavier molecules. Measurements of isotope ratios in mass spectrometers are more robust than measurements of absolute quantities, hence the widespread use of $\delta^{18} \mathrm{O}$. The repeated evaporation and precipitation of water along storm tracks is an example of so-called Rayleigh distillation; the effect is that water precipitated over land is typically much isotopically lighter $(40 \%)$ than ocean water. Land ice, which is formed by accumulated precipitation, typically has $\delta^{18} \mathrm{O}_{\text {ice }}=-40 \%$ [Cronin, 2010].

The $\delta^{18} \mathrm{O}$ of benthic sediment cores has been used for decades as a stratigraphic tool to build relative age models for cores (e.g. Lisiecki and Raymo [2005]). While this 
approach appears to be robust on long time scales, the time it takes for the meltwater isotope anomaly to equilibrate in the global ocean has been modeled [Wunsch and Heimbach, 2008, Siberlin and Wunsch, 2010, Gebbie, 2012, Primeau et al., 2009] to be on the order of thousands of years, potentially introducing errors in calibration when global synchroneity of tracer values is assumed.

While lags between records of $\delta^{18} \mathrm{O}$ complicate its use as a calibration tool, they have been used by several authors to infer properties of the ocean circulation. Skinner and Shackleton [2005] studied cores TR163-31B and MD99-2334K raised from the eastern equatorial Pacific and the Iberian Margin, respectively, and noted a 4000 year lag in the onset of deglaciation (defined as the midpoint of glacial-interglacial $\delta^{18} \mathrm{O}_{c c}$ change) in the two cores. The authors reasoned that this phase difference was too large to be explained by the canonical $\sim 10^{3}$ year mixing time of the ocean or by changes in reservoir ages or record dating uncertainty. Instead, the offset was attributed to in-situ benthic temperature changes in the Pacific and to covarying temperature and $\delta^{18} \mathrm{O}_{s w}$ changes in the Atlantic, suggesting a change in deep water mass source and/or mode of formation at the Atlantic core site.

Wunsch and Heimbach [2008] challenged the conclusion of Skinner and Shackleton [2005] by calculating the propagation of tracer in a steady-state estimate of the modern circulation (ECCO-GODAE) while holding concentration values fixed at regional (basin-sized) surface mixed-layer patches. The time to reach $90 \%$ equilibration was several thousand years in many parts of the intermediate and deep ocean. Regardless of whether the dye patch lay in the North or South Atlantic or South Pacific, deepwater formation sites in the model North Atlantic were principally responsible for the communication of properties from surface to intermediate and deep waters. The model deep and intermediate North Pacific were observed to be the "end of the line" in terms of tracer equilibration times; the North Atlantic had the shortest equi- 
libration times. The authors concluded that a 4000-year lag is not unthinkable given three-dimensional tracer propagation times in the modern circulation, a conclusion that can cautiously be extended to include other, past ocean circulation states with localized regions of deepwater formation remote from core sites.

Primeau et al. [2009] evaluated the use of Dirichlet (concentration) boundary conditions assumed by Wunsch and Heimbach [2008] to simulate the injection of water-borne tracers into the ocean. They showed that tracer equilibration time scales are strongly dependent upon the surface area over which Dirichlet conditions are imposed and noted that the net input of tracer into the interior ocean was a function of the model flow field: the locally-defined $90 \%$ equilibration time of Wunsch and Heimbach [2008] is equivalently the time it takes for $90 \%$ of the water in a local parcel to have traveled through the region of surface forcing. Siberlin and Wunsch [2010] compared Neumann and Dirichlet boundary conditions in a reduced-resolution, offline state-transition version of the ECCO-GODAE flow field and, like Primeau et al. [2009], found reduced equilibration times and Atlantic-Pacific lags in the Neumann boundary case.

Regardless of the boundary condition type that is chosen, the implication from equilibrium time experiments is that changes in tracer properties set in the surface ocean are not observable simultaneously in the global ocean. Depending on the physics of the problem, a spike in tracer concentration at one location may not be observable for centuries or millennia elsewhere. Moreover, sequences of tracer pulses are smoothed and shifted as they move through the ocean, with the effect that information at high frequencies and wavenumbers is subject to path-dependent attenuation. As a result, the problem of inferring remote temporal and spatial patterns in the presence of observational noise is harder at smaller scales, and information will be lost. The distribution of a particular equilibration percentile or the lag between the 
crossing of a specific threshold in two records are special cases of this effect.

In a linear, time-invariant system as was considered by Primeau et al. [2009] and Siberlin and Wunsch [2010], the effect of propagation from some boundary region to an interior location is to smooth tracer signals via convolution with Green function. The Green function (or, in signal processing, the impulse response) is the starting place for a vast literature on signal propagation. Until recently, Green function approaches in paleoceanography centered on so-called transit time distributions (TTDs, Peacock and Maltrud [2006], Rutberg and Peacock [2006]), which are probability distributions for scalar properties (such as ventilation age or tracer concentration) of water parcels; taken in aggregate, TTDs are Green functions. The use of Green functions is outlined in Section 2.3.3.

The properties of observational noise can strongly influence how much can be said about both boundary and flow conditions. Gebbie [2012] applied an inverse approach to the Atlantic-Pacific phase lag discussed in Skinner and Shackleton [2005] and found that a global surface history of $\delta^{18} \mathrm{O}_{c c}$ could be found that agreed with cores TR163-31B and MD99-2334K in a least-squares sense using an estimate of the modern circulation. To arrive at this solution, Gebbie [2012] defined a cost function $J(\mathbf{q})$ which was minimized with respect to the vector $\mathbf{q}$ of time-varying surface tracer concentrations of $\delta^{18} \mathrm{O}_{s w}$ and potential temperature $\theta$. The cost function penalized (1) deviations from a "first guess" deglacial $\delta^{18} \mathrm{O}_{s w}$ and $\theta$ evolution at every surface grid point; (2) rapid transitions in time; and (3) large horizontal tracer gradients at the surface. Inferred surface tracer concentrations resemble modern distribution of $\delta^{18} \mathrm{O}_{s w}$ and $\theta$ over most of the ocean.

Friedrich and Timmermann [2012] compared tracer propagation in coupled GCM simulations with and without a strongly reduced AMOC (intended to mimic H1 conditions) induced by hosing the North Atlantic with $0.5 \mathrm{~Sv}\left(\mathrm{~Sv}=10^{6} \mathrm{~m}^{3} \mathrm{~s}^{-1}\right)$ of fresh- 
water for 2000 years. The authors suggested that transport pathways in the global ocean were sensitive to freshwater forcing and that the steady-state assumptions of Gebbie [2012], Peacock and Maltrud [2006], and others should be revisited.

Previous studies have also evaluated relationships between more than two $\delta^{18} \mathrm{O}$ records. Waelbroeck et al. [2006] considered time series of benthic $\delta^{18} \mathrm{O}_{c c}$ and $\delta^{13} \mathrm{C}_{c c}$ from five sediment cores in the Atlantic, Indian, and Southern Oceans. Simultaneous reductions in $\delta^{18} \mathrm{O}_{c c}$ and $\delta^{13} \mathrm{C}_{c c}$ at a high-latitude Atlantic core site coeval with $\mathrm{H} 1$ and the YD are interpreted as the signature of a poorly-ventilated, brine-generated intermediate water mass. The low $\delta^{13} \mathrm{C}$ properties of this water mass are proposed to be formed by biological fractionation beneath a buoyant fresh-water lid resulting from iceberg melting; the low $\delta^{18} \mathrm{O}$ properties are thought to be due to brine-rejection during sea ice formation of low- $\delta^{18} \mathrm{O}$ glacial meltwater. By comparing with other records, this signal is interpreted as propagating through the ocean, becoming entrained in the Antarctic Circumpolar Current, and mixing with intermediate waters in the Indian Ocean.

Waelbroeck et al. [2011] compiled a network of high-resolution benthic $\delta^{18} \mathrm{O}$ records from the margins of the Atlantic basin with independent age models. The authors concluded that the deglaciation (defined in terms of deviations from a nominal LGM value) is first apparent in waters of roughly $1000 \mathrm{~m}$ and suggested that the later onset of deglaciation in waters deeper than $3000 \mathrm{~m}$ is consistent with a reduction of advection of surface $\delta^{18} \mathrm{O}$ signals in deeper waters during H1. Low benthic $\delta^{13} \mathrm{C}$ and $\delta^{18} \mathrm{O}$ measurements at $\mathrm{H} 1$ were interpreted as northern-sourced brine signals. 


\section{Chapter 2}

\section{Tracer inverse problems in steady}

\section{flows}

This chapter establishes a framework for the inference of time-varying boundary conditions of tracer concentration. Green functions prove to be powerful tools both conceptually and practically for formulating inverse and forward tracer problems. Relating models and observations of tracers in space and time requires careful and consistent bookkeeping; two examples are discussed in detail to illustrate basic practices. The solution relies upon singular value decomposition (SVD) to find the solution to the inverse problem that minimizes misfit with the data in a least-squares sense.

A substantial part of this chapter is dedicated to defining notation and reviewing standard material on inverse methods. The purpose of the chapter is to give the reader some intuition for the paleoceanographic tracer problem (discussed in Chapter 4) through a development of inverse procedures and the consideration of simple models; while the work is original, the motivation here is primarily pedagogical. The 
primary reference for this chapter is Wunsch [2006]; Wunsch et al. [1988] and Wunsch [2002] provide additional examples of simplified tracer models. A vast literature exists on control theory methods and applications (see e.g. Franklin et al. [1997]), and applications to the ocean tracer problem are laid out in Wunsch [1987] and Wunsch [2006].

\subsection{Notation}

Scalar values are written in plain math font; vectors are bold and lower-case; matrices are bold and upper-case. Unless otherwise noted, all vectors are column vectors. A column vector of dimension $N, \mathbf{a} \in \mathbb{R}^{N}$, will be referred to as an $N$-vector. A matrix with $M$ rows and $N$ columns will be referred to as an $M \times N$ matrix. It is convenient in some cases to write matrices and vectors in tensor notation, i.e. $\mathbf{A}=A_{i, j}$, where $i$ indexes rows and $j$ columns; for tensor products, the convention is Einstein summation notation. Individual elements of vectors and matrices are specified using brackets; for example, the element lying in the $i^{\text {th }}$ row and $j^{\text {th }}$ column of a matrix $\mathbf{A}$ is written $[\mathbf{A}]_{i, j}$. Subscripts on scalars denote the index of the scalar value in a matrix, e.g. $\mathbf{x}=\left[x_{1}, x_{2}, \ldots x_{N}\right]^{\top}$. Similarly, subscripts on column vectors denote the column index of the vector within a matrix, e.g $\mathbf{X}=\left[\mathbf{x}_{1}, \mathbf{x}_{2} \ldots \mathbf{x}_{N}\right]$.

Many of the problems addressed in this chapter and Chapter 4 concern a matrix $M \times N($ call it $\mathbf{E})$ describing a linear relationship between the vectors $\mathbf{x}, \mathbf{y}$, and $\mathbf{n}$

$$
\mathbf{y}=\mathbf{E x}+\mathbf{n}
$$

where $\mathbf{y}$ is an $M$-vector whose elements correspond to measurements at multiple spatial locations, $\mathbf{n}$ is an $M$-vector of unknown noise amplitudes measured at the loci 
of observations, and $\mathbf{x}$ is an $L$-vector of unknown values. The rank $K$ of $\mathbf{E}$ is defined as the maximum number of linearly dependent column vectors (or row vectors; they are equivalent) therein. The problem (2.1) is said to be underdetermined if $M<N$, overdetermined for $N<M$, and rank-deficient for $K<\min (M, N)$.

Methods for solving problems having the form $\mathbf{y}=\mathbf{E x}+\mathbf{n}$ are discussed in the following subsection. Solutions or estimates of the true parameters $\mathbf{x}$ are denoted with a tilde, $\tilde{\mathbf{x}}$. The reconstructed data are defined as $\tilde{\mathbf{y}}=\mathbf{E} \tilde{\mathbf{x}}$. The residuals or noise estimates are defined as $\tilde{\mathbf{n}}=\mathbf{y}-\tilde{\mathbf{y}}$.

The covariance matrix of a random vector $\mathbf{a}$ is defined as the dispersion of a about its expectation (taken over multiple realizations of the random vector and denoted by angle brackets),

$$
\mathbf{C}_{\mathbf{a a}}=D^{2}(\mathbf{a}-\langle\mathbf{a}\rangle)=\left\langle(\mathbf{a}-\langle\mathbf{a}\rangle)(\mathbf{a}-\langle\mathbf{a}\rangle)^{\top}\right\rangle
$$

The second moment matrix will be denoted $\mathbf{R}$, e.g.

$$
\mathbf{R}_{\mathrm{aa}}=\left\langle\mathbf{a a}^{\top}\right\rangle
$$

When a process has zero mean, the second moment matrix and the covariance matrix are identical. If an estimate $\tilde{\mathbf{a}}$ of a vector $\mathbf{a}$ has been obtained, then define the uncertainty of the solution to be the dispersion of the estimate about the true value [Wunsch, 2006],

$$
\mathbf{P}=D^{2}(\tilde{\mathbf{a}}-\mathbf{a})=\left\langle(\tilde{\mathbf{a}}-\mathbf{a})(\tilde{\mathbf{a}}-\mathbf{a})^{\top}\right\rangle
$$




\subsection{Machinery: Least Squares and SVD}

This subsection is a recapitulation of material in Wunsch [2006] and other textbooks. It is included to define terms for the convenience of the reader.

\subsubsection{Least Squares}

The least-squares solution to Equation (2.1) is found by minimizing a cost function $J(\mathbf{x})$ equal to the squared solution-data misfit:

$$
\begin{aligned}
J & =\mathbf{n}^{\top} \mathbf{n}=(\mathbf{y}-\mathbf{E x})^{\top}(\mathbf{y}-\mathbf{E x}) \\
\tilde{\mathbf{x}} & =\underset{\mathbf{x}}{\arg \min } J(\mathbf{x})
\end{aligned}
$$

The row-weighted least squares solution includes a weight matrix in the cost function that scales the noise amplitudes,

$$
\begin{aligned}
J & =\mathbf{n}^{\top} \mathbf{W}^{-1} \mathbf{n}=(\mathbf{y}-\mathbf{E x})^{\top} \mathbf{W}^{-1}(\mathbf{y}-\mathbf{E x}) \\
\tilde{\mathbf{x}} & =\underset{\mathbf{x}}{\arg \min } J(\mathbf{x})
\end{aligned}
$$

A common, statistically motivated choice is to set $\mathbf{W}=\mathbf{C}_{\mathbf{n n}}$. Assuming that $\left(\mathbf{E}^{\top} \mathbf{W}^{-1} \mathbf{E}\right)^{-1}$ exists, the solution is given by

$$
\tilde{\mathbf{x}}=\left(\mathbf{E}^{\top} \mathbf{W}^{-1} \mathbf{E}\right)^{-1} \mathbf{E}^{\top} \mathbf{W}^{-1} \mathbf{y}
$$

Additional terms in the cost function can be introduced to impose conditions on the solution in addition to requiring agreement with the data. Consider the modified 
cost function

$$
J(\mathbf{x})=(\mathbf{y}-\mathbf{E x})^{\top} \mathbf{W}^{-1}(\mathbf{y}-\mathbf{E x})+\gamma^{2}\left(\mathbf{x}-\mathbf{x}_{0}\right)^{\top} \mathbf{S}^{-1}\left(\mathbf{x}-\mathbf{x}_{0}\right)
$$

Just as the weight matrix $\mathbf{W}$ scales the relative importance of observations, the second term in the cost function (2.7) can be used to penalize various properties of the solution. The vector $\mathbf{x}_{0}$ is a "first guess"; deviations from the first guess are penalized, and in the absence of constraints from the data, $\tilde{\mathbf{x}}=\mathbf{x}_{0}$. The scalar $\gamma$ determines the relative importance of constraints from the data and from the first guess. The matrix $\mathbf{S}$ defines what kind of deviations from the first guess are penalized. If $\mathbf{S}=\mathbf{I}$, then the solution minimizes the squared difference between $\mathbf{x}$ and $\mathbf{x}_{0}$, a solution known as "tapered" least squares. If $\mathbf{x}_{0}=0$, then the effect is to minimize the variance of $\tilde{\mathbf{x}}$. Alternately, if

$$
\mathbf{S}=\mathbf{H}^{\top} \mathbf{H}
$$

where

$$
\mathbf{H}=\left\{\begin{array}{ccccc}
1 & -1 & 0 & \cdots & 0 \\
0 & 1 & -1 & \cdots & 0 \\
\vdots & \vdots & \vdots & \vdots & \vdots \\
0 & 0 & \cdots & 0 & 1
\end{array}\right\}
$$

then the difference between sequential values of $\tilde{\mathbf{x}}$ about $\mathbf{x}_{0}$ is minimized, yielding a solution that is "smoothed" in the dimension of $\mathbf{x}$.

Cost functions of the form (2.7) can be expressed more succinctly in terms of scaled quantities. Let $\mathbf{A}=\mathbf{A}^{\top / 2} \mathbf{A}^{1 / 2}$ define the Cholesky decomposition of a symmetric, positive-definite matrix $\mathbf{A}$, and similarly denote the decomposition of the inverse as 
$\mathbf{A}^{-1}=\mathbf{A}^{-1 / 2} \mathbf{A}^{-\top / 2}$. Then defining (Wunsch [2006] 2.132)

$$
\begin{aligned}
\mathbf{G}^{\prime} & =\mathbf{W}^{-T / 2} \mathbf{G} \mathbf{S}^{\top / 2} \\
\mathbf{x}^{\prime} & =\mathbf{S}^{-\top / 2} \mathbf{x} \\
\mathbf{x}_{0}^{\prime} & =\mathbf{S}^{-T / 2} \mathbf{x}_{0} \\
\mathbf{n}^{\prime} & =\mathbf{W}^{-T / 2} \mathbf{n} \\
\mathbf{y}^{\prime} & =\mathbf{W}^{-T / 2} \mathbf{y}
\end{aligned}
$$

the cost function (2.7) can equivalently written as

$$
J\left(\mathbf{x}^{\prime}\right)=\left(\mathbf{y}^{\prime}-\mathbf{E}^{\prime} \mathbf{x}^{\prime}\right)^{\top}\left(\mathbf{y}^{\prime}-\mathbf{E}^{\prime} \mathbf{x}^{\prime}\right)+\gamma^{2}\left(\mathbf{x}^{\prime}-\mathbf{x}_{0}^{\prime}\right)^{\top}\left(\mathbf{x}^{\prime}-\mathbf{x}_{0}^{\prime}\right) .
$$

Solutions obtained in this way must be re-scaled. The solution minimizing (2.9) before re-scaling is

$$
\tilde{\mathbf{x}}^{\prime}=\left(\mathbf{E}^{\prime \top} \mathbf{E}^{\prime}+\gamma^{2} \mathbf{I}\right)^{-1}\left(\mathbf{E}^{\prime \top} \mathbf{y}^{\prime}+\gamma^{2} \mathbf{x}_{0}^{\prime}\right)
$$

\subsubsection{Singular value decomposition}

The singular value decomposition (SVD) is written as

$$
\mathbf{E}=\mathbf{U} \boldsymbol{\Lambda} \mathbf{V}^{\top}
$$

where $\mathbf{U}$ and $\mathbf{V}$ are orthonormal matrices whose columns are the eigenvectors of $\mathbf{E E}^{\top}$ and $\mathbf{E}^{\top} \mathbf{E}$, respectively, and $\boldsymbol{\Lambda}$ is a $M \times N$ diagonal matrix with $K$ strictly positive entries $\lambda_{1}, \lambda_{2}, \ldots \lambda_{K}$ in decreasing order along the diagonal. Right-multiplying (2.10) 
by $\mathbf{V}$ gives

$$
\mathbf{E V}=\mathbf{U} \Lambda .
$$

The solution range vectors are the columns $\mathbf{v}_{i}, i=1,2, \ldots K$, of $\mathbf{V}$ for which $\mathbf{E v}_{i} \neq 0$; for $K<N$, the solution nullspace vectors of $\mathbf{E}$ are the $\mathbf{v}_{i} i=K+1, \ldots N$ such that $\mathbf{E v}_{i}=0$. Similarly, left-multiplying (2.10) by $\mathbf{U}^{\top}$ and transposing the equation yields

$$
\mathbf{E}^{\top} \mathbf{U}=\mathbf{V} \Lambda^{\top} .
$$

The data range vectors are the $\mathbf{u}_{i}, i=1,2, \ldots K$, such that $\mathbf{E}^{\top} \mathbf{u}_{i} \neq 0$ and if $K<M$, the data nullspace vectors are the $\mathbf{u}_{i}, i=K+1, \ldots M$, such that $\mathbf{E}^{\top} \mathbf{u}_{i}=0$.

Because the $\mathbf{u}_{i}$ span the space of $\mathbf{y}$ and $\mathbf{n}$ and the $\mathbf{v}_{i}$ span the space of $\mathbf{x}$, it is possible to rewrite the basic relationship $\mathbf{y}=\mathbf{E x}+\mathbf{n}$ in the SVD bases. If the requirement is made, as it is in the derivation of the LS solution, that $\mathbf{u}_{i}^{\top} \mathbf{n}=0, i=$ $1,2, \ldots K$, then the noise estimate or residual $\tilde{\mathbf{n}}=\mathbf{E} \tilde{\mathbf{x}}-\mathbf{y}$ will have a minimum L2 norm and will lie strictly in the data nullspace of $\mathbf{E}$.

The general SVD solutions are

$$
\begin{aligned}
\tilde{\mathbf{x}} & =\sum_{i=1}^{K} \frac{\mathbf{u}_{i}^{\top} \mathbf{y}}{\lambda_{i}} \mathbf{v}_{i}+\sum_{i=K+1}^{N} \alpha_{i} \mathbf{v}_{i} \\
\tilde{\mathbf{y}} & =\mathbf{E} \tilde{\mathbf{x}}=\sum_{i=1}^{K}\left(\mathbf{u}_{i}^{\top} \mathbf{y}\right) \mathbf{u}_{i} \\
\tilde{\mathbf{n}} & =\sum_{i=K+1}^{M}\left(\mathbf{u}_{i}^{\top} \mathbf{y}\right) \mathbf{u}_{i}
\end{aligned}
$$

[Wunsch, 2006]. The nullspace coefficients $\alpha_{i}$ defining $\tilde{\mathbf{x}}$ are unconstrained: there is no information in the data about the structures in the solution nullspace vectors. Equations (2.11) can be written more compactly in matrix form. Define $\mathbf{Q}_{u}$ and $\mathbf{Q}_{v}$ 
to be the matrices whose columns are the nullspace vectors, i.e.

$$
\mathbf{U}=\left\{\begin{array}{ll}
\mathbf{U}_{K} & \mathbf{Q}_{u}
\end{array}\right\}, \quad \mathbf{V}=\left\{\begin{array}{ll}
\mathbf{V}_{K} & \mathbf{Q}_{v}
\end{array}\right\}
$$

where the subscript $K$ denotes a submatrix in which the first $K$ columns (ordered by the $\lambda_{i}$ ) are retained. Then (2.11) is equivalent to

$$
\begin{aligned}
\tilde{\mathbf{x}} & =\mathbf{V}_{K} \Lambda_{K}^{-1} \mathbf{U}_{K}^{\top} \mathbf{y}+\mathbf{Q}_{v} \alpha \\
\tilde{\mathbf{y}} & =\mathbf{U}_{K} \mathbf{U}_{K}^{\top} \mathbf{y} \\
\tilde{\mathbf{n}} & =\mathbf{Q}_{u} \mathbf{Q}_{v}^{\top} \mathbf{y}
\end{aligned}
$$

Setting $\alpha=\mathbf{0}$ minimizes the norm of $\tilde{\mathbf{x}}$ and yields what will be called the particular SVD solution,

$$
\tilde{\mathbf{x}}=\mathbf{V}_{K} \Lambda_{K}^{-1} \mathbf{U}_{K}^{\top} \mathbf{y}
$$

When $\mathbf{E}$ is full rank, the LS solution coincides with the particular SVD solution. Where the least-squares solution does not exist, the SVD solution provides a generalized least-squares approach in terms of the range vectors of $\mathbf{E}$.

The uncertainty $\mathbf{P}$ is defined as the dispersion of $\tilde{\mathbf{x}}$ about the true value. Given that $\langle\mathbf{n}\rangle=0$

$$
\begin{aligned}
\mathbf{P} & \equiv D^{2}(\tilde{\mathbf{x}}-\mathbf{x})=\left\langle(\tilde{\mathbf{x}}-\mathbf{x})(\tilde{\mathbf{x}}-\mathbf{x})^{\top}\right\rangle \\
& =\mathbf{V}_{K} \boldsymbol{\Lambda}_{K}^{-1} \mathbf{U}_{K}^{\top}\left\langle\mathbf{n} \mathbf{n}^{\top}\right\rangle \mathbf{U}_{K} \boldsymbol{\Lambda}_{K}^{-1} \mathbf{V}_{K}^{\top}+\mathbf{Q}_{v}\left\langle\alpha \alpha^{\top}\right\rangle \mathbf{Q}_{v}^{\top} \\
& =\mathbf{C}_{\mathbf{x x}}+\mathbf{Q}_{v}\left\langle\boldsymbol{\alpha} \boldsymbol{\alpha}^{\top}\right\rangle \mathbf{Q}_{v}^{\top}
\end{aligned}
$$

The weights on solution range vectors in Equation (2.11) are determined both by $\mathbf{u}_{i}^{\top} \mathbf{y}$ and $\lambda_{i}$. Very small values of $\lambda_{i}$ that lie in the denominator of elements of $\mathbf{C}_{\mathbf{x x}}$ can 
dramatically increase the solution uncertainty. In many applications it is desirable to reduce $\mathbf{P}$ by truncating the expansion in $\mathbf{v}_{i}$ in at $K^{\prime}<K$, thereby reducing the contribution from $\mathbf{C}_{x x}$ but increasing the nullspace contribution (and biasing the solution). The truncation procedure assumes that there is a range of singular vectors that lie outside of the formal nullspace (for which $\lambda_{i}=0$ ) but which are not useful in the construction of a solution. The selection of $K^{\prime}$ defines the effective nullspace.

The effects of the rank-deficiency of $\mathbf{E}$ on the solution are illustrated by the symmetric resolution matrices $\mathbf{T}_{u}$ and $\mathbf{T}_{v}$. In the absence of observational noise, the solution and estimated data are related to the true parameters and data by $\tilde{\mathbf{x}}=\mathbf{T}_{v} \mathbf{x}$, $\tilde{\mathbf{y}}=\mathbf{T}_{u} \mathbf{y}$, where

$$
\begin{aligned}
& \mathbf{T}_{u}=\mathbf{U}_{K} \mathbf{U}_{K}^{\top} \\
& \mathbf{T}_{v}=\mathbf{V}_{K} \mathbf{V}_{K}^{\top}
\end{aligned}
$$

The resolution matrices will be unity unless $K<M\left(\Longrightarrow \mathbf{T}_{u} \neq \mathbf{I}\right)$ or $K<N$ $\left(\Longrightarrow \mathbf{T}_{v} \neq \mathbf{I}\right)$.

The $i^{t h}$ column (or row) of the solution resolution matrix $\mathbf{T}_{v}$ is the solution $\tilde{\mathbf{x}}_{i}$ obtained when the true parameters are $\mathbf{x}_{i}=\delta_{j, i}$, where $\delta_{i, j}$ is the Kronecker delta function

$$
\delta_{i, j}=\left\{\begin{array}{ll}
1 & i=j \\
0 & i \neq j
\end{array} .\right.
$$

If $\mathbf{x}_{i}=\tilde{\mathbf{x}}_{i}$, then $\mathbf{x}_{i}$ is said to be fully resolved; if not, evidently the solution range vectors of $\mathbf{E}$ are insufficient to represent $\mathbf{x}_{i}$ fully. Similarly, the $i^{\text {th }}$ column of $\mathbf{T}_{u}$ is the expression of a hypothetical observation $\mathbf{y}_{i}=\delta_{j, i}$ in the data range of $\mathbf{E}$; if $\mathbf{y}_{i} \neq \tilde{\mathbf{y}}_{i}$, then the observation is not resolved and the model cannot distinguish changes in the $i^{\text {th }}$ observation from observations distributed as $\tilde{\mathbf{y}}$. The diagonal elements of the 
data resolution matrix $\mathbf{T}_{u}$ may be used to rank the data by their importance to the solution [Wunsch, 2006].

\subsection{Forward and inverse tracer problems}

\subsubsection{Models and controls}

Consider a system with tracer concentration $C(x, y, t)$ described by a two-dimensional, advective-diffusive equation with constant diffusivity $\kappa$ and constant fluid velocities $\mathbf{u}=(u, v)$, on a closed domain $x \in\left[0, L_{x}\right], y \in\left[0, L_{y}\right]$. Define time-dependent tracer concentration boundary conditions at a set of locations $\left\{x_{b}, y_{b}\right\}$ such that the system is defined by

$$
\begin{aligned}
\frac{\partial C}{\partial t}+\mathbf{u} \cdot \nabla C-\kappa \nabla^{2} C & =0 . \\
C(x, y, 0) & =C_{0} \\
C\left(x_{b}, y_{b}, t\right) & =q(t)
\end{aligned}
$$

Equation (2.16) is a model for the two-dimensional evolution of a tracer: it describes the relationships between concentrations at all locations and times. Several additional definitions are helpful here. The quantities $\mathbf{u}$ and $\kappa$ are the parameters of the model. The state of the system is the information internal to the system (as opposed to information about boundary conditions) that is necessary to predict the evolution of the system a small interval of time $\Delta t$ into the future. In the case of Equation (2.16), knowledge of $C(x, y, t)$ and $C\left(x_{b}, y_{b}, t\right)$ permits forward predictions of arbitrary accuracy as $\Delta t \rightarrow 0$. As described above, $C$ is a so-called "passive" tracer, meaning that its concentration does not affect the parameters of the system (unlike, 
for instance, salinity in the ocean, which affects velocity through the density field).

When a physical system is modeled in discrete space, the system can be summarized by the state vector, $\mathbf{c}(t)$, which is the set of elements in the system necessary to describe the state of the system in the discrete model. Consider (2.16) discretized in space using a finite difference approximation, indexing horizontal locations in space by $i=1,2, \ldots I$ and vertical by $j=1,2, \ldots J$ for some integer time step multiple $n$,

$$
\begin{aligned}
\frac{C_{i, j}((n+1) \Delta t)-C_{i, j}(n \Delta t)}{\Delta t}= & \frac{\kappa}{(\Delta x)^{2}}\left(C_{i+1, j}(n \Delta t)-2 C_{i, j}(n \Delta t)+C_{i-1, j}(n \Delta t)\right) \\
& \frac{\kappa}{(\Delta y)^{2}}\left(C_{i, j+1}(n \Delta t)-2 C_{i, j}(n \Delta t)+C_{i, j-1}(n \Delta t)\right) \\
& -\frac{u_{i, j}}{\Delta x}\left(C_{i+1, j}-C_{i, j}\right)-\frac{v_{i, j}}{\Delta y}\left(C_{i, j+1}-C_{i, j}\right) \quad(2.19)
\end{aligned}
$$

At present the details of the discretization are not important; what is notable is that just as $\frac{\partial C}{\partial t}$ was linear in $C$ in equation (2.16), so too is $C(t+1)$ linear in $C(t)$ in equation (2.19). Take $\Delta t=1$ for notational simplicity so that time is an integer. The state vector is

$$
\mathbf{c}(t)=\left[\begin{array}{llllll}
C_{1,1} & C_{2,1} & \ldots & C_{I, 1} & \ldots & C_{I, J}
\end{array}\right]^{\top}
$$

where $\mathbf{c}$ is an $(I * J) \times 1$ column vector. Then (2.19) can be written in the form

$$
\mathbf{c}(t+1)=\mathbf{A c}(t)
$$

where, defining $N \equiv P Q$, the state vector has dimension $N \times 1$ and $\mathbf{A}$ is the $N \times N$ state transition matrix, which is a function of $\mathbf{u}$ and $\kappa$. Since $\mathbf{u}$ and $\kappa$ are constant in time, so is A. Tracer concentrations at boundaries can be enforced by including an additional term $\mathbf{F}(t) \mathbf{q}(t)$ so that

$$
\mathbf{c}(t+1)=\mathbf{A c}(t)+\mathbf{F}(t) \mathbf{q}(t)
$$


Any linear discretized model may be put into the canonical form of Equation 2.20. The elements in the $L$-vector $\mathbf{q}(t)=\left[\begin{array}{llll}q_{1}(t) & q_{2}(t) & \ldots & q_{L}(t)\end{array}\right]^{\top}$ are referred to as controls. The controls are quantities that can be adjusted in order to improve the performance of a system; an aeronautical example is the pitch and roll of an aircraft as it comes in for a landing. The time-varying, $N \times L$ matrix $\mathbf{F}(t)$ will be called the control matrix; it describes how the controls at time $t$ contribute to the state vector at $t+1$.

Say that one wishes to learn how the state will evolve under certain boundary and initial conditions. The forward problem is to specify $\mathbf{F}(t) \mathbf{q}(t)$ and calculate the state vector forward in time from an initial condition $\mathbf{c}(0)$. Equation (2.20) can be rewritten to describe the state vector at any time $t$ explicitly in terms of the initial and boundary conditions:

$$
\mathbf{c}(t)=\mathbf{A}^{t} \mathbf{c}(0)+\mathbf{F}(t-1) \mathbf{q}(t-1)+\mathbf{A F}(t-2) \mathbf{q}(t-2)+\cdots+\mathbf{A}^{t-1} \mathbf{F}(0) \mathbf{q}(0) .
$$

Conversely, often one has some measurements of the left hand side of Equation (2.21) and wants to learn something about quantities on the right hand side: this is the inverse problem. In oceanography, a typical inverse problem is the inference, though observational estimates of tracer concentrations $C_{i, j}$, of the parameters $\mathbf{u}$ and $\kappa$ of a model. In the case where an $M$-vector $\mathbf{y}$ of observations of the physical system is linearly related to the tracer concentrations $\mathbf{c}(t)$, then the inverse problem may be written

$$
\mathbf{y}(t)=\mathbf{B}(t) \mathbf{c}(t)+\mathbf{n}(t)
$$

where $\mathbf{B}(t)$ is an $N \times M$ matrix $^{1}$ (called the data matrix) relating the observations

1 The dimensions of $\mathbf{B}(t)$ and $\mathbf{F}(t)$ will vary in time if the number of observations and controls changes, but one can set the sizes of these matrices to the largest such number at all times $(M$ and 
to the state vector and $\mathbf{n}$ is an $M$-vector of measurement errors or noise. The observations $\mathbf{y}(t)$ in this problem are a nonlinear function of the controls $\mathbf{q}(t)$, the parameters $\mathbf{u}$ and $\kappa$ (through their determination of $\mathbf{A}$ ), and the noise $\mathbf{n}(t)$, and at this point the problem is already complicated.

In practice, the practitioner of inverse methods typically has additional, "prior" information about the boundary conditions and model parameters, and the problem need not be informed strictly by the observations. A major challenge in paleoceanographic research is the specification of prior information for the climate system, which is extremely complex and undersampled. Lagrange multiplier methods [e.g. Dail, 2012, Wunsch, 2006] are able to incorporate prior information from dynamical models and avoid explicitly imposing a flow parameter prior. In the case where the data have the power to constrain model parameters, such techniques can select a candidate set of flow parameters that matches the data while satisfying dynamic constraints (e.g., mass conservation, geostrophy, etc). But numerical models are plagued with problems of their own and cannot provide a firm verdict as to whether or not a certain circulation regime is physically realizable.

For this reason, before turning to Lagrange multiplier methods and considering new physics, a first test of the power of paleoceanographic tracer data is to determine whether or not estimates of the modern circulation fit the data to within error [Gebbie, 2012]. Then the procedure is to assume a set of model parameters as well as covariance estimates $\mathbf{C}_{\mathbf{n n}}$ for the observational noise and $\mathbf{C}_{\mathbf{q q}}$ for the controls and to seek solutions $\tilde{\mathbf{q}}$ for the controls that minimize the model-data misfit (residual) $\tilde{\mathbf{n}}$. This optimization problem - finding the set of boundary conditions that best agrees

$L)$ and substitute rows of zeros to standardize the matrix size. 
with the data in a least-squares sense given a choice of the model parameters that define $\mathbf{A}$ - will be referred to as the inverse boundary condition (IBC) problem.

If a solution to the IBC problem can be found that matches (in a statistical sense, perhaps verified using a goodness of fit test) the prior statistics $\mathbf{C}_{\mathbf{n n}}$ and $\mathbf{C}_{\mathbf{q q}}$, then evidently the modern circulation and choices of initial and boundary conditions are adequate to explain the data. In a sense, this outcome is not desirable from an inference perspective: it implies that the data lack the power to constrain the flow properties [Wunsch, 1987]. When the statistics of the residuals and the observational uncertainty are in disagreement for the best-fit solution $\tilde{\mathbf{q}}$, then there are errors in the model specification, the choice of the parameters $\mathbf{u}$ and $\kappa$, the priors $\mathbf{C}_{\mathbf{n n}}$ and $\mathrm{C}_{\mathrm{qq}}$, or some combination thereof.

All random variables are assumed to be Gaussian. An implication of this assumption is that tracer solutions can technically take real-numbered values (positive or negative). While this approach is at odds with the conventional notion of a tracer there is no such thing as negative concentration - it is a reasonable approximation for a scaled, tracer-like quantity such as $\delta^{18} \mathrm{O}$, or for tracer anomalies about a reference value. It is possible to implement positivity constraints [Gebbie, 2012, Tziperman and Hecht, 1988], but these approaches are not discussed in this work.

\subsubsection{The steady inverse boundary condition problem}

For simplicity, assume that the time step is $\Delta t=1$ and that $t$ is a non-negative integer. The evolution of the state vector is described by Equation (2.21). For the case of constant boundary conditions $\mathbf{q}(t)=\mathbf{q}_{0}$ and $\mathbf{F}(t)=\mathbf{F}_{0}$, Equation (2.21) may 
be written

$$
\mathbf{c}(t)=\mathbf{A}^{t} \mathbf{c}(0)+\left[\sum_{t^{\prime}=0}^{t-1} \mathbf{A}^{t^{\prime}}\right] \mathbf{F}_{0} \mathbf{q}_{0}
$$

Here it is useful to note a property of the transition matrix used in the paleoceanographic application in Chapter 4. In that system, tracer enters the system state (defined to be the ocean below the mixed layer) and then is said to exit the system state as it upwells; this upwelling is prescribed by the state transition matrix. For this reason, the following discussion treats transition matrices that also "lose" tracer by fluxing it out of the state. For any such state transition matrix, $\mathbf{A}^{t} \rightarrow \mathbf{0}$ as $t \rightarrow \infty^{2}$ and the sum of matrix powers converges to a matrix with non-negative elements defined as $\mathbf{A}_{\infty} \equiv \sum_{t^{\prime}=1}^{\infty} \mathbf{A}^{t^{\prime}}$. Physically, this corresponds to the system's "forgetting" long-past values (including initial conditions).

In the time-asymptotic case when there is no influence from initial conditions, $\mathbf{c}(t)$ is constant in time and one can define $\lim _{t \rightarrow \infty} \mathbf{c}(t) \equiv \mathbf{c}_{\infty}$; then 2.23 is

$$
\mathbf{c}_{\infty}=\mathbf{A}_{\infty} \mathbf{F}_{0} \mathbf{q}_{0}
$$

Alternately, using that as $t \rightarrow \infty, \mathbf{c}(t)=\mathbf{c}(t+\Delta t)=\mathbf{c}_{\infty}$, the canonical form $(2.20)$ becomes

$$
\mathbf{c}_{\infty}=\mathbf{A c}_{\infty}+\mathbf{F}_{0} \mathbf{q}_{0}
$$

\footnotetext{
${ }^{2}$ Proof: Gershgorin's circle theorem states that for a matrix $A$ with any eigenvalue $\lambda,\left|\lambda-A_{i, i}\right| \leq$ $\sum_{i \neq j}\left|A_{i, j}\right|$. Fully connected state transition matrices that lose tracer have the property that $\sum_{i=1}^{n} A_{i, j}<1 \forall j$, implying that $\left|\lambda-A_{i, i}\right| \leq \sum_{i \neq j}\left|A_{i, j}\right|<1-\left|A_{i, i}\right| \Longrightarrow|\lambda|<1$ [Golub and Van Loan, 2012, Giraudo]. This is true for any $\lambda$, and because the eigenvalues of $\mathbf{A}$ all obey $|\lambda|<1$, $\mathbf{A}^{t} \rightarrow \mathbf{0}$ as $t \rightarrow \infty$
} 
From (2.25),

$$
\mathbf{c}_{\infty}=(\mathbf{I}-\mathbf{A})^{-1} \mathbf{F}_{0} \mathbf{q}_{0}
$$

evidently $\mathbf{A}_{\infty}=(\mathbf{I}-\mathbf{A})^{-1}$ and the existence of the inverse is guaranteed by the convergence of the sum $\mathbf{A}_{\infty} \equiv \sum_{t^{\prime}=1}^{\infty} \mathbf{A}^{t^{\prime}}$. Equation (2.26) is the time-asymptotic solution to the forward problem under fixed boundary conditions.

In the inverse boundary condition problem, one typically has noisy observations representative of only a subset of the state vector. Using the steady, forward model solution (2.26) in (2.22), ignoring the effects of initial conditions, and assuming a steady data matrix $\mathbf{B}(t)=\mathbf{B}_{0}$, the inverse problem for the inference of steady boundary conditions can be written

$$
\mathbf{y}_{0}=\mathbf{B}_{0}(\mathbf{I}-\mathbf{A})^{-1} \mathbf{F}_{0} \mathbf{q}_{0}+\mathbf{n} .
$$

The steady inverse problem can be written in the standard form $\mathbf{y}=\mathbf{E x}+\mathbf{n}$ discussed in Section 2.2, where $\mathbf{E}=\mathbf{B}_{0}(\mathbf{I}-\mathbf{A})^{-1} \mathbf{F}_{0}$ is an $M \times L$ matrix that describes $M$ equations (one for each observation) in $L$ unknowns (the controls). Solutions to this problem could be sought, for example, by minimizing a cost function

$$
\begin{aligned}
J\left(\mathbf{q}_{0}\right) & =\left(\mathbf{y}-\mathbf{B}_{0}(\mathbf{I}-\mathbf{A})^{-1} \mathbf{F}_{0} \mathbf{q}_{0}\right)^{\top}\left(\mathbf{y}-\mathbf{B}_{0}(\mathbf{I}-\mathbf{A})^{-1} \mathbf{F}_{0} \mathbf{q}_{0}\right) \\
\tilde{\mathbf{q}_{0}} & =\underset{\mathbf{q}_{0}}{\arg \min } J\left(\mathbf{q}_{0}\right)
\end{aligned}
$$

bringing row and column weights to bear if appropriate. Depending on the size and rank of $\left(\mathbf{B}_{0}(\mathbf{I}-\mathbf{A})^{-1} \mathbf{F}_{0} \mathbf{q}_{0}\right)$, the problem could have a solution nullspace, a data nullspace, or both. 


\subsubsection{The time-varying inverse boundary condition problem: Green functions and the whole-domain formulation}

The forward evolution of the state vector is fully specified by (2.20) and a choice of initial and boundary conditions. The inverse problem is made more tractable by reformulating the forward problem in terms of Green functions, which are introduced here.

In the previous section, the transient effects of initial conditions were ignored and the time-asymptotic state vector was determined by the choice of time-invariant boundary conditions. Now consider the complementary case where (2.20) is a pure initial value problem with $\mathbf{q}(t)=0$ such that Equation (2.21) becomes

$$
\mathbf{c}(t)=\mathbf{A}^{t} \mathbf{c}(0)
$$

Following Wunsch [2002, section 5], define a time-dependent $N$-vector indexed by position $j$,

$$
\mathbf{g}_{j}\left(t_{0}, t\right)=\mathbf{A}^{t} \mathbf{g}_{j}\left(t_{0}, t_{0}\right), t \geq t_{0}
$$

where $\left[\mathbf{g}_{j}\left(t_{0}, t_{0}\right)\right]_{i}=\delta_{i j}$ is a vector consisting of zeros at all indices $i \neq j$ and 1 at $i=j$. The time-dependent vector $\mathbf{g}_{j}\left(t_{0}, t\right)$ is the numerical Green function. It describes the time evolution of the state vector from the initial condition $\mathbf{g}_{j}\left(t_{0}, t_{0}\right)$.

If the dimension of the state space is $N$, then one may define $N$ Green function vectors $\mathbf{g}_{j}\left(t_{0}, t\right)$ for $j=1,2, \ldots N$. These vectors may be grouped to form a $N \times N$ Green function matrix defined as

$$
\mathbf{G}\left(t_{0}, t\right)=\left[\begin{array}{llll}
\mathbf{g}_{1}\left(t_{0}, t\right) & \mathbf{g}_{2}\left(t_{0}, t\right) & \ldots & \mathbf{g}_{N}\left(t_{0}, t\right)
\end{array}\right]
$$


and obeying

$$
\begin{gathered}
\mathbf{G}\left(t_{0}, t+1\right)=\mathbf{A G}\left(t_{0}, t\right), \mathbf{G}\left(t_{0}, t_{0}\right)=\mathbf{I} \\
\Longrightarrow \mathbf{G}\left(t_{0}, t\right)=\mathbf{A}^{t}
\end{gathered}
$$

Because the flow has been assumed to be stationary in time, the Green function $\mathbf{g}_{j}\left(t_{0}, t\right)$ describing the contribution to the state vector at time $t$ from a unitary concentration in the $j^{\text {th }}$ box at any time $t_{0} \geq 0$ can be written strictly in terms of the difference between $t_{0}$ and $t$,

$$
\mathbf{G}\left(t_{0}, t\right)=\mathbf{G}\left(t-t_{0}\right) .
$$

To avoid notational ambiguity, the stationary Green function matrix corresponding to a lag $\tau$ will be indexed by a subscript rather than written as a function of time, i.e.

$$
\mathbf{G}_{t-t_{0}} \equiv \mathbf{G}\left(t-t_{0}\right)= \begin{cases}\mathbf{A}^{\left(t-t_{0}\right)} & t \geq t_{0} \\ 0 & t<t_{0}\end{cases}
$$

Having defined this notation, the general forward problem (2.21) can readily be recast in terms of the Green functions by substituting according to (2.29):

$$
\begin{aligned}
\mathbf{c}(t)= & \mathbf{G}_{t} \mathbf{c}(0)+\mathbf{G}_{0} \mathbf{F}(t-1) \mathbf{q}(t-1)+\mathbf{G}_{1} \mathbf{F}(t-2) \mathbf{q}(t-2)+\ldots \\
& +\mathbf{G}_{t-1} \mathbf{F}(0) \mathbf{q}(0), .
\end{aligned}
$$

This expression can be written as a convolution,

$$
\mathbf{c}(t)=\mathbf{G}_{t} c(0)+\sum_{t^{\prime}=0}^{t-1} \mathbf{G}_{t^{\prime}} \mathbf{F}\left(t-1-t^{\prime}\right) \mathbf{q}\left(t-1-t^{\prime}\right) .
$$


Equation (2.30) may be written for different times $t=1,2, \ldots \tau$ in a set of simultaneous equations in matrix-vector form,

$$
\begin{aligned}
{\left[\begin{array}{c}
\mathbf{c}(1) \\
\mathbf{c}(2) \\
\vdots \\
\mathbf{c}(\tau)
\end{array}\right]=} & {\left[\begin{array}{c}
\mathbf{G}_{1} \\
\mathbf{G}_{2} \\
\vdots \\
\mathbf{G}_{\tau}
\end{array}\right] \mathbf{c}(0) } \\
& {\left[\begin{array}{cccc}
\mathbf{G}_{0} & \mathbf{0} & \ldots & \mathbf{0} \\
\mathbf{G}_{1} & \mathbf{G}_{0} & \ldots & \mathbf{0} \\
\vdots & & \ddots & \\
\mathbf{G}_{\tau} & \mathbf{G}_{\tau-1} & \ldots & \mathbf{G}_{0}
\end{array}\right]\left[\begin{array}{cccc}
\mathbf{F}(0) & \mathbf{0} & \ldots & \mathbf{0} \\
\mathbf{0} & \mathbf{F}(1) & \ldots & \mathbf{0} \\
\vdots & \vdots & \ddots & \vdots \\
\mathbf{0} & \mathbf{0} & \ldots & \mathbf{F}(\tau)
\end{array}\right]\left[\begin{array}{c}
\mathbf{q}(0) \\
\mathbf{q}(1) \\
\vdots \\
\mathbf{q}(\tau-1)
\end{array}\right] }
\end{aligned}
$$

The concatenated vectors of $\mathbf{c}(t)$ and $\mathbf{q}(t)$ are indexed in both space and time and are referred to as whole-domain state and control vectors. All whole-domain vectors and matrices will be denoted with an overbar:

$$
\overline{\mathbf{c}} \equiv\left[\begin{array}{c}
\mathbf{c}(1) \\
\mathbf{c}(2) \\
\vdots \\
\mathbf{c}(\tau)
\end{array}\right], \quad \overline{\mathbf{q}} \equiv\left[\begin{array}{c}
\mathbf{q}(0) \\
\mathbf{q}(1) \\
\vdots \\
\mathbf{q}(\tau-1)
\end{array}\right]
$$

Note that while $\overline{\mathbf{c}}$ and $\overline{\mathbf{q}}$ span the same number of time steps $\tau$, the actual times are offset by one. This offset is a reflection of the statement in (2.20) that the state vector at a time $t$ only "sees" the effects of the controls from time $t-1$ and earlier. 
It is also convenient to define the whole-domain block matrices

$$
\begin{gathered}
\overline{\mathbf{G}}_{0}=\left[\begin{array}{c}
\mathbf{G}_{1} \\
\mathbf{G}_{2} \\
\vdots \\
\mathbf{G}_{\tau}
\end{array}\right], \overline{\mathbf{G}}=\left[\begin{array}{cccc}
\mathbf{G}_{0} & \mathbf{0} & \ldots & \mathbf{0} \\
\mathbf{G}_{1} & \mathbf{G}_{0} & \ldots & \mathbf{0} \\
\vdots & & \ddots & \\
\mathbf{G}_{\tau} & \mathbf{G}_{\tau-1} & \ldots & \mathbf{G}_{0}
\end{array}\right] \\
\overline{\mathbf{F}}=\left[\begin{array}{cccc}
\mathbf{F}(0) & \mathbf{0} & \ldots & \mathbf{0} \\
\mathbf{0} & \mathbf{F}(1) & \ldots & \mathbf{0} \\
\vdots & \vdots & \ddots & \vdots \\
\mathbf{0} & \mathbf{0} & \ldots & \mathbf{F}(\tau)
\end{array}\right]
\end{gathered}
$$

so that the whole-domain formulation of the forward problem is simply

$$
\overline{\mathbf{c}}=\overline{\mathbf{G}}_{0} \mathbf{c}(0)+\overline{\mathbf{G F}} \overline{\mathbf{q}} .
$$

Equation (2.32) is exactly equivalent to the statement of the forward problem in Equation (2.21). In the control theory literature, matrices of the form $\overline{\mathbf{G}}_{0}$ are known as "controllability" matrices when the controls are the initial conditions $\mathbf{c}(0) ; \overline{\mathbf{G}}$ describes $\tau$ simultaneous controllability problems.

The time-varying inverse problem can also be written in whole-domain form. Substituting the convolution form of state evolution observations (2.30) into the definition of the inverse problem (2.22) yields

$$
\begin{aligned}
\mathbf{y}(t)= & \mathbf{B}(t) \mathbf{G}_{t} \mathbf{c}(0) \\
& +\mathbf{B}(t) \sum_{t^{\prime}=0}^{t-1} \mathbf{G}_{t^{\prime}} \mathbf{F}\left(t-1-t^{\prime}\right) \mathbf{q}\left(t-1-t^{\prime}\right)+\mathbf{n}(t) .
\end{aligned}
$$


Define the whole-domain block data matrix

$$
\overline{\mathbf{B}}=\left[\begin{array}{cccc}
\mathbf{B}(1) & \mathbf{0} & \ldots & \mathbf{0} \\
\mathbf{0} & \mathbf{B}(2) & \ldots & \mathbf{0} \\
\vdots & \vdots & \ddots & \vdots \\
\mathbf{0} & \mathbf{0} & \ldots & \mathbf{B}(\tau)
\end{array}\right]
$$

and the noise and data vectors as

$$
\overline{\mathbf{n}}=\left[\begin{array}{c}
\mathbf{n}(1) \\
\mathbf{n}(2) \\
\vdots \\
\mathbf{n}(\tau)
\end{array}\right], \overline{\mathbf{y}}=\left[\begin{array}{c}
\mathbf{y}(1) \\
\mathbf{y}(2) \\
\vdots \\
\mathbf{y}(\tau)
\end{array}\right]=\overline{\mathbf{B}}\left[\begin{array}{c}
\mathbf{c}(1) \\
\mathbf{c}(2) \\
\vdots \\
\mathbf{c}(\tau)
\end{array}\right]+\overline{\mathbf{n}}
$$

Equation (2.33) in whole-domain matrix form is then

$$
\overline{\mathbf{y}}=\overline{\mathbf{B}} \overline{\mathbf{G}}_{0} \mathbf{c}(0)+\overline{\mathbf{B}} \overline{\mathbf{G}} \overline{\mathbf{F}} \overline{\mathbf{q}}+\overline{\mathbf{n}}
$$

Equation (2.34) is the most general and compact form of the IBC problem for steady flow. The focus of this thesis is on understanding and solving special cases of this equation. Note that when effects from initial conditions are neglected, the equation has the canonical form $\mathbf{y}=\mathbf{E x}+\mathbf{n}$.

All of the information about how tracer boundary conditions are propagated to the loci of observations is specified in the matrix $\overline{\mathbf{B}} \overline{\mathbf{G}} \overline{\mathbf{F}}$, which is unwieldy in large problems. To gain some physical intuition for this operator, consider the block submatrices of $\overline{\mathbf{B}} \overline{\mathbf{G}} \overline{\mathbf{F}}$ in the simplified case where $\mathbf{B}(t)=\mathbf{B}_{0}$ and $\mathbf{F}(t)=\mathbf{F}_{0}$. Assuming that $\mathbf{B}(t)$ and $\mathbf{F}(t)$ are constant requires that observational loci and the influence of 
controls on the state vector are time-invariant, respectively. Then each block submatrix of $\overline{\mathbf{B}} \overline{\mathbf{G}} \overline{\mathbf{F}}$ is of the form $\mathbf{B}_{0} \mathbf{G}_{t} \mathbf{F}_{0}$.

Define the function $\zeta_{i j}(t)=\left[\mathbf{B}_{0} \mathbf{G}_{t} \mathbf{F}_{0}\right]_{i j}, t=0,1, \ldots, \tau$ to be the impulse response. The impulse response $\zeta_{i j}(t)$ describes the evolution of tracer at the observational locations specified in the $i^{\text {th }}$ row of $\mathbf{B}_{0}$ in the case when the control values are

$$
q_{k}(t)= \begin{cases}1 & t=0 \text { and } k=j \\ 0 & \text { otherwise }\end{cases}
$$

Writing the convolution form of the inverse problem (2.33) in index form, neglecting initial conditions and substituting the definition of the impulse response, yields

$$
\begin{aligned}
y_{i}(t)-n_{i}(t) & =\sum_{t^{\prime}=0}^{t-1} \zeta_{i j}\left(t^{\prime}\right) q_{j}\left(t-1-t^{\prime}\right) \\
& =\zeta_{i j}(t) \star q_{j}(t-1)
\end{aligned}
$$

where $\star$ denotes convolution in time. Equation $(2.35)$ treats $\mathbf{q}(t)$ as a sequence of impulses and $\mathbf{B} \mathbf{c}(t)=\mathbf{y}(t)-\mathbf{n}(t)$ (call this the observable signal) as the difference of sequential impulse responses and noise. Convolution relationships of this form are familiar from the study of linear, time invariant filters; evidently observable signals are filtered versions of the control signals and the kernel of the filter is the impulse response (see also Rutberg and Peacock [2006]). Viewed in this light, $\overline{\mathbf{B}} \overline{\mathbf{G}} \overline{\mathbf{F}}$ is a filtering operator and the inverse boundary problem is a deconvolution problem.

Consideration of (2.35) in the Fourier domain will be useful for understanding the differences between observable and control signals, as well as the null space of the deconvolution problem. Define the discrete Fourier transform (DFT) of a function 
$f(t)$ to be

$$
\hat{f}(s)=\sum_{m=-\infty}^{\infty} f(t) e^{-2 \pi i s \Delta t} .
$$

Applying the discrete forms of the convolution and shift theorems (the latter to account for the time step offset between $q_{j}(t-1)$ and $\zeta_{i j}(t)$ in equation (2.35)) yields

$$
\hat{y}_{i}(s)-\hat{n}_{i}(s)=e^{2 \pi i s \Delta t} \hat{\zeta}_{i j}(s) \hat{q}_{j}(s)
$$

which states that the DFT of the $j^{\text {th }}$ control signal, $\hat{q}_{j}(s)$ and of the $i^{\text {th }}$ observable signal $\hat{y}_{i}(s)$, are related multiplicatively in frequency space by the complex transfer function, $\hat{\zeta}_{i j}(s)$. The gain $\left|\hat{\zeta}_{i j}(s)\right|$ is the amplitude attenuation of the convolution operator and $\arg \left(\hat{\zeta}_{i j}(s)\right)$ is the associated phase change.

In practice the computation of the matrices in Equation (2.34) can be prohibitive. As written in Equation (2.31), it is necessary to run $\tau$ steps of the forward model $L$ times in order to compute $\overline{\mathbf{G}}$. Recall that $\mathbf{G}_{t-t_{0}}=\mathbf{A}^{\left(t-t_{0}\right)}$ (Eq. (2.29)). Whenever $M<L$, it is more efficient (but exactly equivalent) to compute the transpose of each of the block matrices in $\overline{\mathbf{B}} \overline{\mathbf{G}} \overline{\mathbf{F}}$ using

$$
\mathbf{B}\left(t_{1}\right) \mathbf{G}_{t_{2}} \mathbf{F}\left(t_{3}\right)=\mathbf{B}\left(t_{1}\right) \mathbf{A}^{t_{2}} \mathbf{F}\left(t_{3}\right)=\left(\mathbf{F}\left(t_{3}\right)^{\top} \mathbf{A}^{\top t_{2}} \mathbf{B}\left(t_{1}\right)^{\top}\right)^{\top},
$$

where $t_{1}, t_{2}$ and $t_{3}$ are three integer times corresponding to one of the block submatrices and $\mathbf{A}^{\top t_{2}}$ is the $t_{2}^{t h}$ power of the "adjoint" model $\mathbf{A}^{\top}$. Then $\overline{\mathbf{B}} \overline{\mathbf{G}} \overline{\mathbf{F}}$ is computed by running $\tau$ steps of the adjoint model $\mathbf{A}^{\top} M$ times, once for each row of $\mathbf{B}$. The adjoint model can be thought of as running the forward model backwards in time and transporting tracer away from the data locations.

In the numerical simulations run to generate $\overline{\mathbf{B}} \overline{\mathbf{G}} \overline{\mathbf{F}}$ in Chapter 4 , it is more numerically stable to calculate the forward model response to a step function tracer 
pulse than to a delta function. The response to the step function is the cumulative sum of the impulse response and is called the Heaviside response

$$
Z_{i j}(t) \equiv \sum_{t^{\prime}=0}^{t} \zeta_{i j}\left(t^{\prime}\right)
$$

which describes the evolution of tracer concentrations specified in the $i^{t h}$ row of $\mathbf{B}$ in the case where the control values are

$$
q_{k}(t)= \begin{cases}1 & t \geq 0 \text { and } k=j \\ 0 & \text { otherwise }\end{cases}
$$

This is a case of steady boundary conditions. If a limit is taken in $t$,

$$
\begin{aligned}
Z_{i j}(t) & \equiv \sum_{t^{\prime}=0}^{t} \zeta_{i j}\left(t^{\prime}\right) \\
& =\sum_{t^{\prime}=0}^{t}\left[\mathbf{B}_{0} \mathbf{G}_{t^{\prime}} \mathbf{F}_{0}\right]_{i, j} \\
\lim _{t \rightarrow \infty} Z_{i j}(t) & =\left[\mathbf{B}_{0}\left(\sum_{t^{\prime}=0}^{\infty} \mathbf{G}_{t^{\prime}}\right) \mathbf{F}_{0}\right]_{i j} \\
& =\left[\mathbf{B}_{0} \mathbf{A}_{\infty} \mathbf{F}_{0}\right]_{i j}
\end{aligned}
$$

recovering the steady solution derived in 2.3.2.

\subsection{Inference from tracers in a four-box model}

In this section, the general discrete advective-diffusive equation (2.19) is considered in the simplified context of a four-box model. This model has been constructed to mimic some of the features of the more complex tracer transport model used in Chapter 4. Two of the four boxes in the model are designated "control boxes" and the 
other two constitute the system state. Fluid volume bearing tracer enters the system state from the control boxes at each time step, and an equal volume of fluid exits the system state in order to satisfy volume conservation. The two control boxes are analogous to the mixed layer in the model used in Chapter 4. When fluid is upwelled into the ocean mixed layer, its tracer properties may be modified by surface climate properties and thus not conserved.

\subsubsection{Model setup}

Consider the model depicted in Figure 2.1, in which there are four homogeneous fluid reservoirs labeled $1-4$, each with a time-varying scalar property $C_{i}(t) \in \mathbb{R}$. Take this property to be the (homogeneous) tracer concentration (tracer mass per volume) in the reservoir ${ }^{3}$. Such a model might represent fluxes between porous membranes in a filtration system, chambers in a heart, or ocean basins. The first objective is to describe the forward evolution of the model state in the canonical form (2.20).

The parameters of the model are the reservoir volumes $V_{i}$ and the volume flux $J_{i j} \geq 0$ between reservoirs $i$ and $j$. Volume fluxes are specified to be positive to permit bidirectional exchanges between reservoirs. The volume fluxes can be representative of advective processes, diffusive properties, or both. As a stability requirement, the discrete time step of the model, $\Delta t$, is chosen so that boxes cannot be completely refilled in a single time step:

$$
\frac{\sum_{i} J_{i j}}{V_{j}} \Delta t<1 \forall j .
$$

\footnotetext{
${ }^{3}$ Physically, concentration can only take positive values, but inference under positivity constraints requires special procedures that are not essential to the present discussion. It suffices to assume that values here have been shifted and rescaled to take a range of positive and negative values.
} 
Equivalently, the time step is required to be less than the tracer residence time $\tau_{j}=$ $\frac{V_{j}}{\sum_{i} J_{i j}}$ in every box.

Boxes 1 and 2 are defined as boundary reservoirs; the concentrations $C_{1}(t)$ and $C_{2}(t)$ are the boundary conditions of the forward tracer problem and the control vector is $\mathbf{q}(t)=\left[\begin{array}{ll}C_{1}(t), & C_{2}(t)\end{array}\right]^{\top}$. At each time step, the boundary conditions are set to a prescribed value by a mechanism exterior to the model setup. The model state is specified by the concentrations of boxes 3 and 4 (the interior reservoirs) and the state vector is $\mathbf{c}(t)=\left[\begin{array}{ll}C_{3}(t), & C_{4}(t)\end{array}\right]^{\top}$.

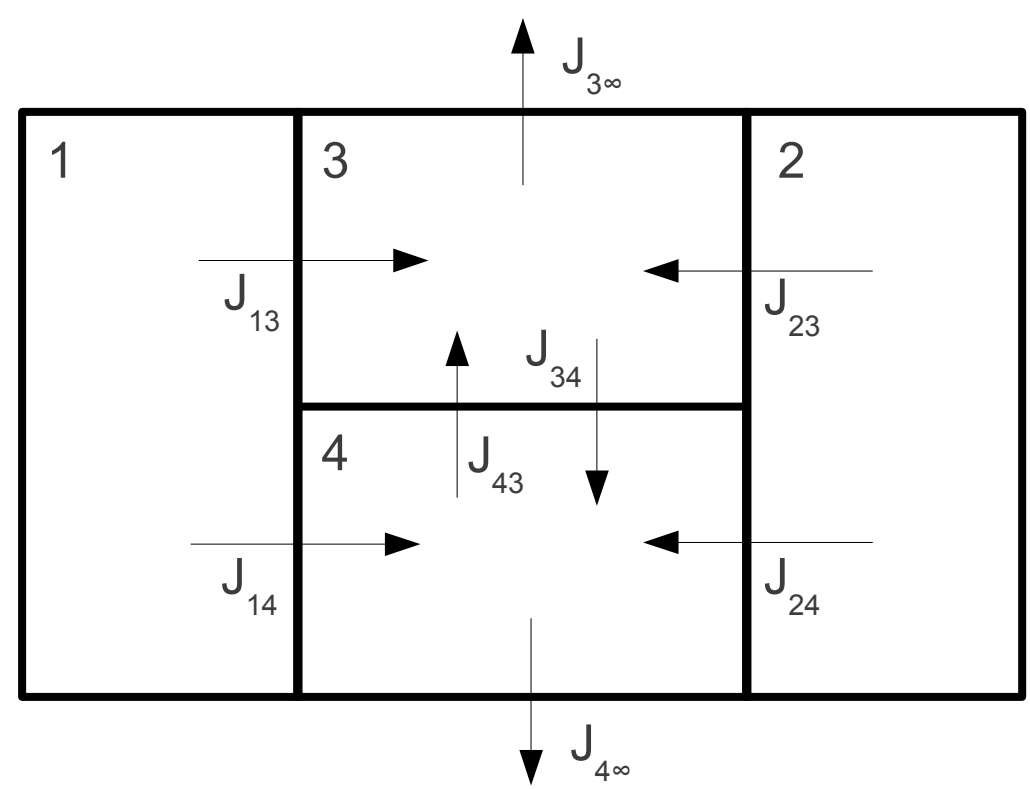

Figure 2.1: A box model with four reservoirs related by constant volume flux rates. The subscript $\infty$ denotes that the flow is out of the system.

Define the set of box indices corresponding to the state and controls as $\mathscr{S}$ and $\mathscr{C}$, respectively. For a box model of arbitrary size, the equation of volume conservation for all $i \in \mathscr{S}$ is

$$
\sum_{j \in \mathscr{S} \cup \mathscr{C}}\left\{J_{i j}-J_{j i}\right\}+J_{i \infty}=0
$$


and the equations for tracer mass conservation are

$$
V_{i} \frac{\left(C_{i}(t+\Delta t)-C_{i}(t)\right)}{\Delta t}=\sum_{j \in \mathrm{S} \cup \mathrm{C}}\left\{C_{j}(t) J_{j i}-C_{i}(t) J_{i j}\right\}-C_{i}(t) J_{i \infty}
$$

Combining Equations (2.38) and (2.39) to eliminate the $J_{i \infty}$ yields a closed form for the evolution of the tracer in terms of interior and boundary boxes,

$$
C_{i}(t+\Delta t)=\sum_{j \in \mathrm{S} \cup \mathrm{C}}\left\{\left[1-\frac{J_{i j} \Delta t}{V_{i}}\right] C_{i}(t)+\frac{J_{j i} \Delta t}{V_{i}} C_{j}(t)\right\} .
$$

The forward tracer evolution equation is readily written in the canonical form of Equation (2.20) using the definitions of the state and control vectors $\mathbf{c}(t)$ and $\mathbf{q}(t)$. The state transition matrix is [Wunsch et al., 1988]

$$
\begin{aligned}
A_{i i} & =1-\sum_{j \in \mathrm{S} \cup \mathrm{C}} \frac{J_{i j} \Delta t}{V_{j}} \\
A_{i j} & =\sum_{j \in \mathrm{S}} \frac{J_{j i} \Delta t}{V_{j}}, i \neq j
\end{aligned}
$$

and $\mathbf{F}$ is

$$
F_{i j}=\sum_{j \in \mathrm{C}} \frac{J_{j i} \Delta t}{V_{j}}
$$

The state evolution equation in the four-box case can then be written in terms of the 
state transition matrix $\mathbf{A}$ and control matrix $\mathbf{F}$ according to (2.39):

$$
\begin{aligned}
\underbrace{\left[\begin{array}{c}
C_{3}(t+\Delta t) \\
C_{4}(t+\Delta t)
\end{array}\right]}_{\mathbf{c}(t+\Delta t)}= & \underbrace{\left[\begin{array}{cc}
1-\frac{J_{13}+J_{23}+J_{43}}{V_{3}} \Delta t & \frac{J_{43}}{V_{3}} \Delta t \\
\frac{J_{34}}{V_{4}} \Delta t & 1-\frac{J_{14}+J_{24}+J_{34}}{V_{4}} \Delta t
\end{array}\right]}_{\mathbf{A}} \underbrace{\left[\begin{array}{c}
C_{3}(t) \\
C_{4}(t)
\end{array}\right]}_{\mathbf{c}(t)} \\
& \underbrace{\Delta t\left[\begin{array}{cc}
\frac{J_{13}}{V_{3}} & \frac{J_{23}}{V_{3}} \\
\frac{J_{14}}{V_{4}} & \frac{J_{24}}{V_{4}}
\end{array}\right]}_{\mathbf{F}} \underbrace{\left[\begin{array}{c}
C_{1}(t) \\
C_{2}(t)
\end{array}\right]}_{\mathbf{q}(t)}
\end{aligned}
$$

\subsubsection{The steady inverse boundary condition problem}

The steady IBC problem is the inference of steady controls $\mathbf{q}_{0}$ using noisy measurements of $\mathbf{c}_{\infty}$. Define observations $\mathbf{y}_{0}$ to be samples of the steady state vector plus a noise component,

$$
\mathbf{y}_{0}=\mathbf{B}_{0} \mathbf{c}_{\infty}+\mathbf{n}
$$

In the case where $M$ observations of the state vector are available, $\mathbf{y}_{0}$ and $\mathbf{n}$ are $M$-vectors, $\mathbf{c}_{\infty}$ is a 2 -vector, $\mathbf{F}_{0}$ is $2 \times 2$, and $\mathbf{B}_{0}$ is $2 \times M$.

Recall that the statement of the steady inverse problem (2.27) is

$$
\mathbf{y}_{0}=\left[\mathbf{B}_{0}(\mathbf{I}-\mathbf{A})^{-1} \mathbf{F}_{0}\right] \mathbf{q}_{0}+\mathbf{n}
$$

As discussed in 2.3.2, (2.27) has the canonical form $\mathbf{y}=\mathbf{E x}+\mathbf{n}$. If one has $a$ priori information about the noise amplitude and/or some desirable properties of the solution, then the inverse problem may be row- and column-weighted accordingly (see 
(2.8)) and the scaled problem written as

$$
\begin{aligned}
& \mathbf{y}_{0}^{\prime}=\mathbf{E}^{\prime} \mathbf{q}_{0}^{\prime}+\mathbf{n}^{\prime} \\
& \mathbf{E}^{\prime}=\mathbf{W}^{-\frac{T}{2}} \mathbf{B}_{0}(\mathbf{I}-\mathbf{A})^{-1} \mathbf{F}_{0} \mathbf{S}^{-\frac{T}{2}} \\
& \mathbf{q}_{0}^{\prime}=\mathbf{S}^{-\top / 2} \mathbf{q}_{0} \\
& \mathbf{n}^{\prime}=\mathbf{W}^{-\top / 2} \mathbf{n} \\
& \mathbf{y}_{\mathbf{0}}^{\prime}=\mathbf{W}^{-\top / 2} \mathbf{y}_{0}
\end{aligned}
$$

By specifying the solution and data nullspaces, the singular values and vectors of the $\mathbf{E}^{\prime}$ matrix provide an important diagnostic of the power of the data to resolve features in the controls. Specifically, the scaled left singular vectors $\mathbf{U}^{\prime}$ describe the structures in $\mathbf{y}$ that are projected onto the right singular vectors $\mathbf{V}^{\prime}$ in the range contribution (the $\left.\lambda_{i} \neq 0, i=1,2, \ldots K\right)$ to the SVD solution (Equation (2.11))

$$
\tilde{\mathbf{q}}_{0}^{\prime}=\sum_{i=1}^{K} \frac{\mathbf{u}_{i}^{\prime \top} \mathbf{y}^{\prime}}{\lambda_{i}} \mathbf{v}_{i}^{\prime}+\sum_{i=K+1}^{N} \alpha_{i} \mathbf{v}_{i}^{\prime}
$$

Moreover, small singular values are an indicator of structures (the corresponding singular vectors) that cannot be well resolved in the data and solution (particularly, they are sensitive to noise in the data). These ill-resolved structures can be eliminated from the solution by defining an effective nullspace $K^{\prime}<K$. The following four examples discuss the inverse problem in different configurations.

\section{Case 1: Full-rank}

Set $J_{13}=J_{14}=J_{24}=J_{43}=1, J_{34}=J_{23}=0, \Delta t=1$, and $V_{3}=V_{4}=10$. Thus the boundary reservoirs contribute equally to the interior boxes and half of the flux out of box 4 is into box 3 (the other half leaves the system). Stipulating that 
$\mathbf{B}_{0}=\mathbf{W}^{-1}=\mathbf{S}^{-1}=\mathbf{I}$ yields

$$
\begin{gathered}
\mathbf{A}=\left[\begin{array}{cc}
0.8 & 0.1 \\
0 & 0.8
\end{array}\right], \mathbf{F}_{0}=\left[\begin{array}{cc}
0.1 & 0 \\
0.1 & 0.1
\end{array}\right],(\mathbf{I}-\mathbf{A})^{-1}=\left[\begin{array}{cc}
5 & 2.5 \\
0 & 5
\end{array}\right] \\
\mathbf{E}=\overline{\mathbf{W}}^{-\frac{T}{2}} \mathbf{B}_{0}(\mathbf{I}-\mathbf{A})^{-1} \mathbf{F}_{0} \overline{\mathbf{S}}^{-\frac{T}{2}}=\left[\begin{array}{cc}
0.75 & 0.25 \\
0.5 & 0.5
\end{array}\right]
\end{gathered}
$$

In this case, $\mathbf{W}^{-1} \mathbf{B}_{0},(\mathbf{I}-\mathbf{A})^{-1}, \mathbf{F}_{0}$, and $\mathbf{S}^{-1}$ are all full-rank. The singular value decomposition $\mathbf{E}=\mathbf{U} \boldsymbol{\Lambda} \mathbf{V}^{\top}$ is, after rounding to two significant figures,

$$
\mathbf{U}=\left[\begin{array}{cc}
-0.75 & -0.66 \\
-0.66 & 0.75
\end{array}\right], \quad \Lambda=\left[\begin{array}{cc}
1.0 & 0 \\
0 & 0.24
\end{array}\right], \mathbf{V}=\left[\begin{array}{cc}
-0.86 & -0.50 \\
-0.50 & 0.86
\end{array}\right]
$$

The inverse problem is formally just determined ("formally" in the sense that there is an equal number of observations and controls; the presence of noise makes the problem underdetermined) and full rank. There are no null spaces and the resolution matrices are unitary.

\section{Case 2: Null space from $B$ and $\mathrm{W}^{-1}$}

The matrices $\mathbf{B}$ and $\mathbf{W}^{-1}$ are both row weighting matrices, but they represent different aspects of the inverse problem: $\mathbf{B}$ describes the structure of the observations or linear problem (and may not be square), and $\mathbf{W}^{-1}$ is chosen to be the inverse of the noise covariance matrix. Consider the case in which the noise amplitude in Box 4 is ten times larger than that in Box 3: $\sigma_{4}=10, \sigma_{3}=1$ : 


$$
\mathbf{B}=\left[\begin{array}{ll}
1 & 0 \\
0 & 1
\end{array}\right], \mathbf{W}=\left[\begin{array}{cc}
1 & 0 \\
0 & 100
\end{array}\right], \mathbf{W}^{-\frac{\tau}{2}}=\left[\begin{array}{cc}
1 & 0 \\
0 & 0.1
\end{array}\right], \mathbf{S}=\left[\begin{array}{ll}
1 & 0 \\
0 & 1
\end{array}\right]
$$

Using the same values for $\mathbf{F}_{0}$, and $\mathbf{A}$ as above, the scaled matrix $\mathbf{E}^{\prime}$ is

$$
\mathbf{E}^{\prime}=\mathbf{W}^{-\frac{\tau}{2}} \mathbf{B}_{0}(\mathbf{I}-\mathbf{A})^{-1} \mathbf{F}_{0} \mathbf{S}^{-\frac{\tau}{2}}=\left[\begin{array}{cc}
0.75 & 0.25 \\
0.05 & 0.05
\end{array}\right]
$$

and its singular value decomposition is

$$
\mathbf{U}^{\prime}=\left[\begin{array}{cc}
-1.0 & -0.08 \\
-0.08 & 1.0
\end{array}\right], \quad \mathbf{\Lambda}^{\prime}=\left[\begin{array}{cc}
0.79 & 0 \\
0 & 0.03
\end{array}\right], \quad \mathbf{V}^{\prime}=\left[\begin{array}{cc}
-0.95 & -0.32 \\
-0.32 & 0.95
\end{array}\right]
$$

This problem is mathematically full-rank, but the second singular value $\lambda_{2}$ is very small relative to the first. The first singular vectors project a quantity dominated by the (relatively low-noise) observation of $C_{3}$ onto a mode $\mathbf{v}_{1}$ that attributes concentrations in the boundary boxes in approximate proportion to their flux into the system.

\section{Case 3: The null space from $F$}

What happens to the solution if the influence of one of the boundary reservoirs becomes very small? Consider the case where $J_{13}$ is reduced to $10^{-1}$. Then

$$
\mathbf{A}=\left[\begin{array}{cc}
0.8 & 0.1 \\
0 & 0.89
\end{array}\right], \quad \mathbf{F}=\left[\begin{array}{cc}
0.1 & 0 \\
0.1 & 0.01
\end{array}\right],(\mathbf{I}-\mathbf{A})^{-1}=\left[\begin{array}{cc}
5.0 & 5.0 \\
0 & 9.9
\end{array}\right]
$$




$$
\mathbf{E}=\mathbf{W}^{-\frac{\tau}{2}} \mathbf{B}_{0}(\mathbf{I}-\mathbf{A})^{-1} \mathbf{F}_{0} \mathbf{S}^{-\frac{\tau}{2}}=\left[\begin{array}{cc}
0.95 & 0.05 \\
0.91 & 0.01
\end{array}\right]
$$

The rows of $\mathbf{E}$ are nearly linearly dependent and the SVD of $\mathbf{E}$ is now

$$
\mathbf{U}=\left[\begin{array}{cc}
-0.71 & -0.71 \\
-0.71 & 0.71
\end{array}\right], \quad \Lambda=\left[\begin{array}{cc}
1.3 & 0 \\
0 & 0.03
\end{array}\right], \quad \mathbf{V}=\left[\begin{array}{cc}
-1.0 & -0.07 \\
-0.07 & 1.0
\end{array}\right]
$$

If the small value $\lambda_{2}$ is set to 0 , then inference of the second control $C_{2}$ lies in the effective nullspace of the inverse problem. This makes physical sense - very little information about $C_{2}$ is present in the system relative to the noise amplitude. Since only $C_{1}$ is observable in the system, gradients between the observable boxes are unobservable, and the vector proportional to such gradients lies in the left nullspace $\mathbf{u}_{2}$. The resolution matrices are

$$
\mathbf{T}_{u}=\left[\begin{array}{ll}
0.53 & 0.50 \\
0.50 & 0.48
\end{array}\right], \quad \mathbf{T}_{v}=\left[\begin{array}{cc}
0.99 & 0.07 \\
0.07 & 0.005
\end{array}\right]
$$

The fact that similar values lie on the diagonal of $\mathbf{T}_{u}$ means that the two measurements have roughly equal importance in determining the solution. However, the data have very different resolving power for the two control values: the control vector $\mathbf{q}=[1,0]^{\top}$ will be reconstructed as $\tilde{\mathbf{q}}=[0.99,0.07]^{\top}$, but the control vector $\mathbf{q}=[0,1]^{\top}$ will be reconstructed as $\tilde{\mathbf{q}}=[0.07,0.005]^{\top}$, a dramatic reduction in solution norm that does not preserve any of the structure of the true controls.

\section{Case 4: The null space from $\mathrm{S}^{-1}$}

In most cases, one has a priori information about the boundary conditions that does not come from the data. In the paleoceanographic case, placing prior constraints 
on surface distributions of tracer perturbations is complicated and involves complicated mixed-layer processes. In the four box model, structures in the controls can be thought of as originating from a prescribed covariance between $C_{1}$ and $C_{2}$. For example, specify that $C_{1}$ and $C_{2}$ are known to covary highly:

$$
\begin{aligned}
\mathbf{S} & =\mathbf{R}_{\mathbf{q q}}=\left[\begin{array}{cc}
1 & 0.99 \\
0.99 & 1
\end{array}\right] \\
\Longrightarrow \mathbf{S}^{-1} & =\left[\begin{array}{cc}
5.3 & -4.7 \\
-4.7 & 5.3
\end{array}\right], \mathbf{S}^{-\frac{T}{2}}=\left[\begin{array}{cc}
2.3 & -2.1 \\
0 & 1.0
\end{array}\right]
\end{aligned}
$$

and that all other matrices are the same as in the full-rank case. Then

$$
\begin{gathered}
\mathbf{E}^{\prime}=\mathbf{W}^{-\frac{T}{2}} \mathbf{B}_{0}(\mathbf{I}-\mathbf{A})^{-1} \mathbf{F}_{0} \mathbf{S}^{-\frac{T}{2}}=\left[\begin{array}{cc}
0.75 & 0.78 \\
0.5 & 0.57
\end{array}\right] \\
\mathbf{U}^{\prime}=\left[\begin{array}{cc}
-0.82 & -0.57 \\
-0.57 & 0.82
\end{array}\right], \mathbf{\Lambda}^{\prime}=\left[\begin{array}{cc}
1.3 & 0 \\
0 & 0.03
\end{array}\right], \mathbf{V}^{\prime}=\left[\begin{array}{cc}
-0.68 & -0.73 \\
-0.73 & 0.68
\end{array}\right]
\end{gathered}
$$

A solution that includes the second set of singular vectors will have a substantially higher uncertainty because of the relatively small associated singular value. This greater uncertainty reflects the high covariance specified a priori by $\mathbf{S}$ - by stipulating that gradients between the controls are unlikely, the presence of any gradient in the solution is inferred as likely due to error.

\subsubsection{The time-varying inverse boundary value problem}

The time-varying inverse boundary value problem is the inference of $\mathbf{q}(t)$ using 
noisy measurements of $\mathbf{c}(t)$. Here, the purpose is to consider the information lost as a tracer signal (or time series) in the boundary regions of the four box models propagates into the interior boxes.

As in the steady tracer case, set the time step $\Delta t=1, J_{13}=J_{14}=J_{24}=J_{43}=1$, $J_{34}=J_{23}=0$, and $V_{3}=V_{4}=10, \mathbf{B}=\mathbf{I}$ so that

$$
\mathbf{A}=\left[\begin{array}{cc}
0.8 & 0.1 \\
0 & 0.8
\end{array}\right], \quad \mathbf{F}=\left[\begin{array}{cc}
0.1 & 0 \\
0.1 & 0.1
\end{array}\right], \quad \mathbf{B}=\left[\begin{array}{ll}
1 & 0 \\
0 & 1
\end{array}\right]
$$

Consider the case in which the initial conditions are known and specify $\mathbf{c}(0)=\mathbf{0}$ and $\tau=20$. The statement of the weighted, time-varying inverse problem is

$$
\overline{\mathbf{y}}^{\prime}=\left[\overline{\mathbf{W}}^{-\frac{\mathrm{T}}{2}} \overline{\mathbf{B G F S}}^{-\frac{\mathrm{T}}{2}}\right] \overline{\mathbf{q}}^{\prime}+\overline{\mathbf{n}}^{\prime}
$$

The matrix choices specified in (2.46) yield the four impulse responses $\zeta_{i j}(t)=$ $[\mathbf{B G}(t) \mathbf{F}]_{i j}$ plotted in Figure 2.2. $\zeta_{41}(t)$ and $\zeta_{42}(t)$ - the responses observed in Box 4 to impulses in Boxes 1 and 2 - are exactly the same: a spike of 0.1 at $t=1$, followed by an exponential decay. The responses $\zeta_{31}(t)$ and $\zeta_{32}(t)$ of Box 3 to impulses in the two control boxes are qualitatively different. An impulse in Box 1 is observed in Box 3 at $t=1$ as a spike to 0.1 followed by a gradual decay, but an impulse in Box 2 is observed in Box 3 as a smooth time series with the first nonzero value at $t=2$ and maximum values at $t=5$ and $t=6$.

The features in the impulse responses are readily rationalizable. The impulse response at $t=1$ is determined by the matrix $\mathbf{F}$; for instance, in the cases of $\zeta_{41}$ and $\zeta_{42}, 1 / 10$ of the volume of Box 4 is replaced by fluxes from boxes 1 and 2 at each time step. The phase lag before onset in $\zeta_{32}(t)$ arises because the signal from Box 2 can only get to Box 3 by going through Box 4, which takes a model time step. The signal 

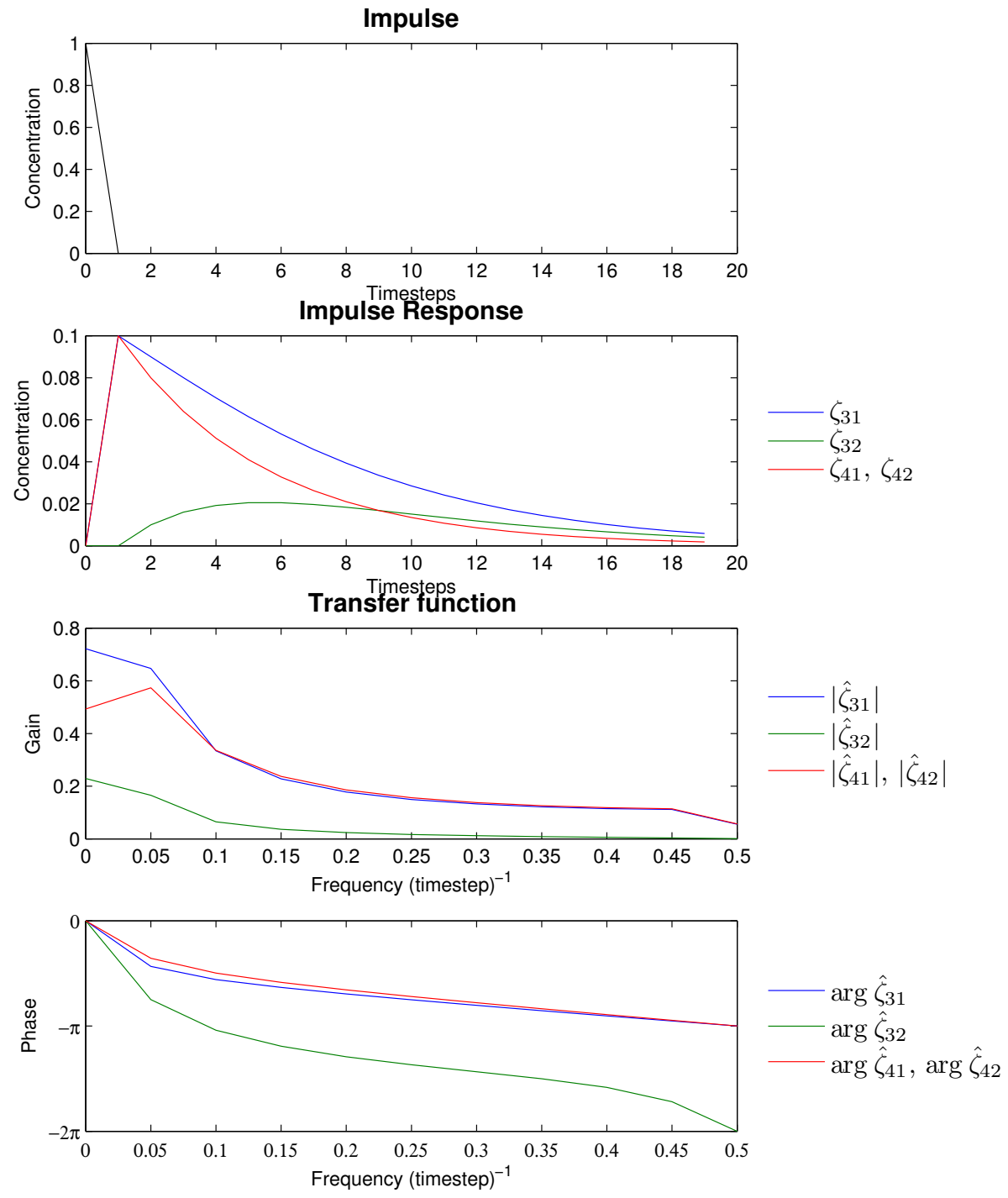

Figure 2.2: The discrete-time impulse response $\zeta_{i j}(t)$ describes the evolution of tracer concentration at the $i^{\text {th }}$ state element in response to an "impulse" in the $j^{\text {th }}$ control element. Panel (a) shows the impulse, which is 0 at all times except $t=0$, when it is 1. Panel (b) shows the impulse response in each of the two state boxes due to impulses in the two control boxes. $\zeta_{41}(t)$ and $\zeta_{42}(t)$ are the same. Measurable tracer concentrations in the state boxes are smoothed and phase-shifted relative to the impulse. The transfer function is the Fourier transform of the impulse response; the real and imaginary parts of the transfer function are plotted in (c) and (d) and describe the attenuation and lag of the initial impulse experienced in each state box as a function of frequency, respectively. 
from Box 1 in Box 3 appears smooth because the flux of tracer from Box 1 into Box 3 compensates the exponential decay in Box 4. The latter statement also explains why Box 3 has a fatter "tail" of concentration at large times: new tracer is constantly being fluxed into Box 3 from Box 4 even as both slowly approach their equilibrium values of zero concentration at $t=\infty$. Indeed, because tracer exchange between any two boxes is described by a convolution, downstream boxes will always have smoother responses to boundary impulses, and the phase lag increases monotonically downstream at all frequencies along a one-dimensional pathway.

The amplitude (gain) and argument (phase shift) of the transfer function (the Fourier transform of the impulse response) relates the frequency content of control signals (those in boxes 1 and 2) and observable signals (3 and 4). All frequencies are attenuated; higher frequencies are attenuated more than lower frequencies. The 0 frequency (the mean) is the integral over the impulse response (equivalently, the Heaviside response) over the times calculated; as the time over which the impulse response is calculated grows to infinity, $\arg \hat{\zeta}_{i j}(0) \rightarrow A_{\infty i j}$. The phase shifts are smoothly varying curves between the mean and the Nyquist frequency (half the sampling frequency, the highest frequency resolvable by the time step). The mean phase shift is 0 ; the Nyquist phase shift is $\pi$ or $2 \pi$ depending on whether the first nonzero impulse response falls on an odd or even time step, respectively.

What are the importance of the gain and phase shift for inference? In the steady inference problem discussed in Section (2.3.2), when the flux from one control reservoir was relatively small, the singular vector associated with the inference of the control reservoir concentration was very small, yielding greater uncertainty. The $\tau$ time step time-varying problem with $L$ control boxes is equivalent to a $L \times \tau$-box steady problem, where the objective is to infer the amplitude of each Fourier component from each control box, and the gain is equivalent to the flux out of each box. Thus, 
in analogy with the steady case, when the gain is small for a particular frequency, the uncertainty is larger. In general, the signal-to-noise ratio of the observations and the uncertainty of the inferred solutions both depend upon frequency.

Although the gain lies in the Fourier domain rather than the SVD domain, the two bases span the same set of time-varying functions, and features that are ill-resolved in one basis will be ill-resolved in the other. This statement is borne out by the SVD plotted in Figure 2.3. In that figure, the left and right singular vectors of the timedependent problem resemble harmonic functions, especially for lower SVD indices. Because of the connection to linear filters, the Fourier basis is an intuitive way to consider the null space of the IBC deconvolution problem. (In some applications, the deconvolution problem is solved in the Fourier domain, a procedure known as spectral division.)

Another nullspace in the time-dependent problem arises from the destructive interference of control signals in the system state. This matter is addressed further in Gebbie [2012].

Changes in phase are important for the interpretation of paleoceanographic tracer records (see the discussion of Skinner and Shackleton [2005] and ensuing papers in the Chapter 1) and a natural question to ask is how robustly the phases of signals can be inferred given observational uncertainty. However, the phase uncertainty is a complicated function of the observational uncertainty (as well as the chronological uncertainty, which is ignored here altogether) and a discussion is outside the scope of this thesis at present. The useful lesson is that phases are shifted between the control 

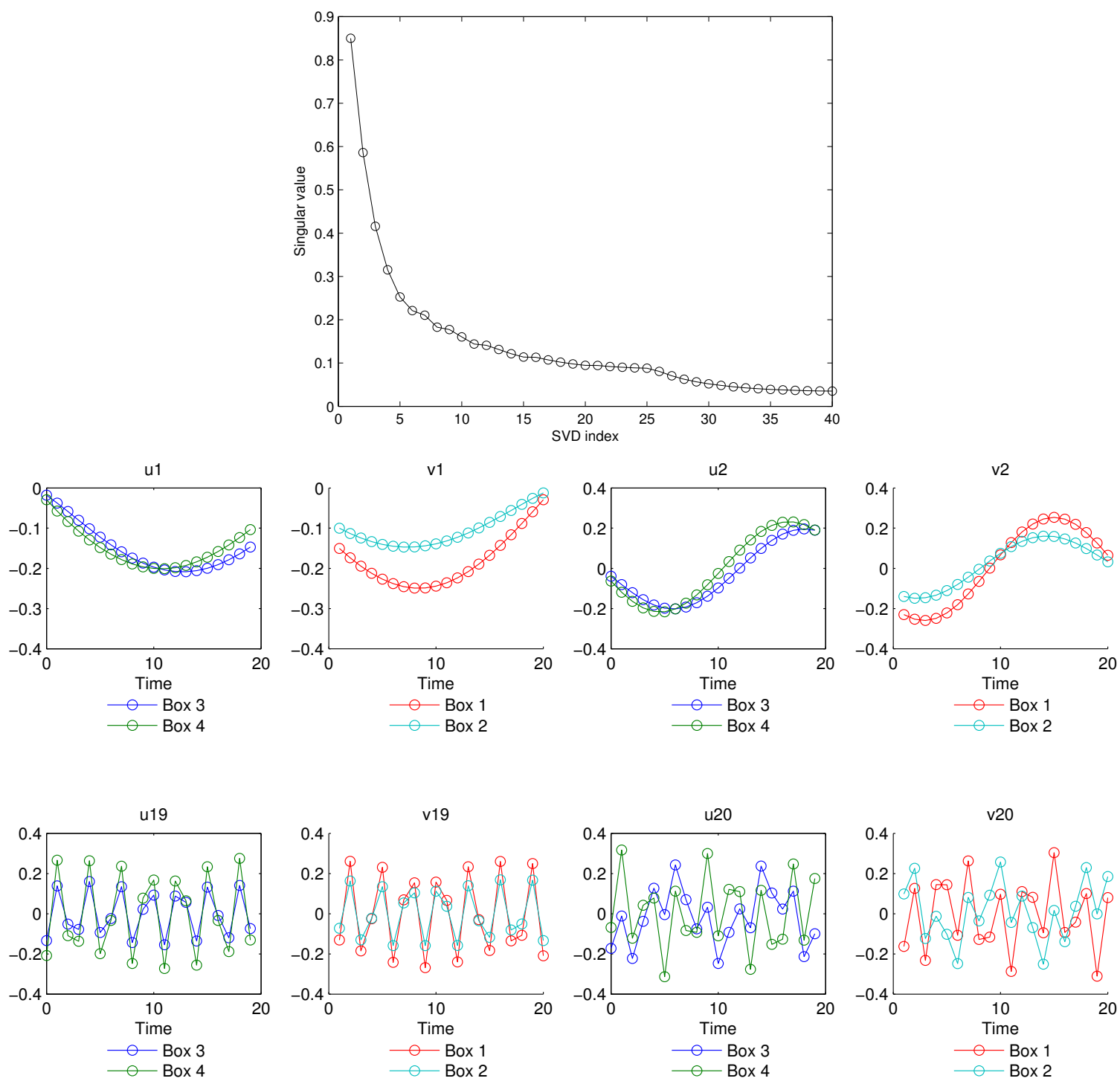

Figure 2.3: Top: Singular values for the time-dependent four-box tracer inverse boundary problem decay rapidly with SVD index from a few high values to values roughly an order of magnitude smaller. Bottom: Left and right singular vector pairs corresponding to SVD indices 1, 2, 19, and 20 plotted as time series for each box. Left singular vectors show structures in the observable boxes 3 and 4 , while right singular vectors $v_{i}$ describe structures in the control boxes 1 and 2 . The offset in time axes results from the one time-step delay in control inference. The lower amplitude of Box 3 relative to Box 4 in the right singular vectors is the result of the smaller flux into the observable boxes from Box 3 . 
and observable signals.

\subsubsection{A comment on null spaces in the four-box model}

The weighted, time-varying inverse problem is

$$
\overline{\mathbf{y}}^{\prime}=\left[\overline{\mathbf{W}}^{-\frac{\mathrm{T}}{2}} \overline{\mathbf{B}} \overline{\mathbf{G}} \overline{\mathbf{F}} \overline{\mathbf{S}}^{-\frac{\mathrm{T}}{2}}\right] \overline{\mathbf{q}}^{\prime}+\overline{\mathbf{n}}^{\prime}
$$

As was demonstrated in the steady and time-varying problems, vanishing singular values in the matrix $\mathbf{E}=\overline{\mathbf{W}}^{-\frac{T}{2}} \overline{\mathbf{B G F S}}^{-\frac{T}{2}}$ can arise from vanishing singular values in any of its five matrix factors $\left(\overline{\mathbf{W}}^{-\frac{T}{2}}, \overline{\mathbf{B}}, \overline{\mathbf{G}}, \overline{\mathbf{F}}, \overline{\mathbf{S}}^{-\frac{T}{2}}\right)$. Each of these matrices is a linear operator representing a physical process in the inverse problem, and each can attenuate or destroy spatiotemporal structures in tracer concentration. Structures that are destroyed in this way lie in the nullspace of the operator; that is, for an $M \times N$ operator $\mathbf{E}$ with rank $K<N$, the structures lie in the space spanned by the right nullspace vectors of the operator, $\mathbf{v}_{K+1}, \ldots \mathbf{v}_{N}$, for which $\mathbf{E v}_{i}=\mathbf{0}$. Specifically:

1. The right null space of $\overline{\mathbf{W}}^{-\frac{T}{2}}$ corresponds to signal features that are present at the loci of observations but are dwarfed by noise.

2. The right null space of $\overline{\mathbf{B}}$ corresponds to features that are present in the system state but which are not observed.

3. The right null space of $\overline{\mathbf{G}}$ corresponds to features that are destroyed by the advection and diffusion of tracer signals along fluid transport pathways en route to loci in the system state.

4. The right null space of $\overline{\mathbf{F}}$ corresponds to features that are present in the controls but which are not communicated to the system state. 
5. The right null space of $\overline{\mathbf{S}}^{\frac{T}{2}}$ corresponds to features that are a priori "undesirable" (aesthetically, statistically, or otherwise) in the control fields.

\subsection{Inference of steady flow: Models of Gebbie and Huybers [2010, 2012]}

Previous sections in this chapter described tools for the inference of tracer boundary conditions under steady flow assumptions. Errors in inferred boundary conditions arise whenever modeled flow patterns and rates deviate from the modeled system. In the ocean, deriving even a statistically steady estimate of flow that is constrained by observations is a highly nontrivial task. This section highlights the two such efforts that are used in tandem in the deglacial problem studied in Chapter 4.

Building on previous water mass decomposition studies [e.g., Tomczak Jr, 1981, Johnson, 2008], Gebbie and Huybers [2010] (henceforth GH10) devised a tracer inversion procedure dubbed "Total Matrix Intercomparision" (TMI) to constrain a watermass pathways model using climatological tracer observations. First note that the tracer concentration of a fluid parcel in the ocean interior can be expressed as a sum

of contributions from $N$ source volumes, each constituting a mass fraction $0 \leq m_{i} \leq 1$ of the parcel and having a concentration $C_{i}$ :

$$
C=\sum_{i=1}^{N} m_{i} C_{i}+\Delta C
$$

(GH10, Eq 1) where $\Delta C$ is a term accounting for interior tracer sources and sinks. If the parcel is taken to be a rectangular grid box in a three-dimensional model, then 
Equation (2.47) can be written in terms of the mass contributions and concentrations of the six neighboring grid boxes adjacent to the faces of the grid box whose indices are offset by 1:

$$
\begin{aligned}
\Delta C_{i j k}= & C_{i j k}-m_{1} C_{i+1, j k}-m_{2} C_{i-1, j k}-m_{3} C_{i, j+1, k} \\
& -m_{4} C_{i, j-1, k}-m_{5} C_{i j, k+1}-m_{6} C_{i, j, k-1}, \quad i, j, k \in I
\end{aligned}
$$

where $I$ is the set of interior grid boxes (GH10, Eq. 15).

While Equation (2.48) is intuitive, solving a system of such equations for all interior points is a nonlinear problem because both the $m_{i}$ and $C_{i j k}$ represent unknown quantities. TMI is a two-step procedure that finds approximate solutions to Equation (2.48) by linearizing about the local (grid box-scale) tracer flux problem and using the inferred $m_{i}$ and $\Delta C_{i j k}$ in the global problem. For a single grid box, observations of $M$ tracers $C^{1}, C^{2}, \ldots C^{M}$ can be written as a system of equations of the form $C^{j}=\sum_{i=1}^{6}\left(C_{i}^{j}+\Delta e_{i}^{j}\right) m_{i}+n^{j}$, where both $\Delta e_{i}^{j}$ and $n^{j}$ are observational noise processes. In vector form,

$$
\left[\begin{array}{c}
C^{1} \\
C^{2} \\
\vdots \\
C^{M}
\end{array}\right]=\left(\left[\begin{array}{cccc}
C_{1}^{1} & C_{2}^{1} & \ldots & C_{6}^{1} \\
C_{1}^{2} & C_{2}^{2} & \ldots & C_{6}^{2} \\
\vdots & \vdots & \vdots & \vdots \\
C_{1}^{M} & C_{2}^{M} & \ldots & C_{6}^{M}
\end{array}\right]+\left[\begin{array}{cccc}
\Delta e_{1}^{1} & \Delta e_{2}^{1} & \ldots & \Delta e_{6}^{1} \\
\Delta e_{1}^{2} & \Delta e_{2}^{2} & \ldots & \Delta e_{6}^{2} \\
\vdots & \vdots & \vdots & \vdots \\
\Delta e_{1}^{M} & \Delta e_{2}^{M} & \ldots & \Delta e_{6}^{M}
\end{array}\right]\right)\left[\begin{array}{c}
m_{1} \\
m_{2} \\
\vdots \\
m_{6}
\end{array}\right]+\left[\begin{array}{c}
n^{1} \\
n^{2} \\
\vdots \\
n^{M}
\end{array}\right] .
$$

Equation (2.49) is appended with equations of mass conservation $\left(\sum_{i=1}^{6} m_{i}=1\right)$ and tracer non-conservation (the $\Delta C_{i j k}$ ) and solved using a nonlinear tapered least squares approach subject to $m_{i}>0 \forall i$. Nonconservative tracer effects emerge from nutrient proxies, which are constrained in GH10 by a stoichiometric ratio, and by unresolved 
processes such as abyssal overflows. The effect is a constrained, simultaneous minimization of the $n^{j}$ and $\Delta e_{i}^{j}$ and inference of $m_{i}$ and $\Delta C_{i j k}$.

Having solved the local problems, Equation (2.48) may be written simultaneously for all interior grid points in matrix form as

$$
\mathrm{Ac}=\mathrm{d}
$$

where the vector $\mathbf{c}$ describes the concentration of each tracer in every grid box in the model state (a generalization of the "state vector" discussed earlier in this chapter) and $\mathbf{A}$ is a matrix of the $m_{i}$ that describes ocean transport pathways. The vector $\mathbf{d}$ is a vector of source terms $\Delta C_{i j k}$ due either to nonconservative behavior in interior grid points (from remineralization, etc.) or from the domain boundary, where parcels are reset by external forcing (e.g., isotopic exchange with the atmosphere). The boundary Green functions are the columns, corresponding to boundary grid boxes, of the matrix $\mathbf{A}^{-1}$.

In GH10, the relative contributions of 2806 surface $4 \times 4$ degree grid boxes to the boxes in the interior ocean were estimated by inverting observations of distributions of potential temperature, salinity, phosphate, nitrate, and oxygen in the modern ocean from hydrographic transects (WOCE and previous measurements, Gouretski and Koltermann [2004]) and the gridded $\delta^{18} \mathrm{O}_{s w}$ product of LeGrande and Schmidt [2006]. The resulting steady-state solution agrees with the tracer measurements, some of which date to the 1950s, within estimated observational error. A "twin" experiment using artificial data generated by adding noise to GCM output and a reconstruction with artificial noise added to the observations both suggested that the TMI reconstructions have less than 5\% error. Gebbie and Huybers [2011] performed a similar experiment but using a higher-resolution $(2 \times 2$ degree $)$ domain and showed the 
pathways to be consistent with the GEOSECS [Kroopnick, 1985] $\delta^{13} \mathrm{C}$ distribution, which was not used to constrain TMI.

Estimating flow rates requires additional steps. Gebbie and Huybers [2012] (hereafter GH12) sought upper and lower bounds as well as a mean estimate of the ages (the time since ventilation) of water parcels in the interior ocean using the GLODAP gridded dataset of pre-anthropogenic radiocarbon, which is based on hydrographic sections, and a correction for bomb radiocarbon using measured potential alkalinity [Rubin and Key, 2002, Key et al., 2004]. Similarly as in TMI procedure, GH12 first performed a local inversion for the residence time $\tau$ of every grid box and then solved a system of tracer equations for the advection of a mean age tracer $a$,

$$
a=\sum_{i=1}^{6} m_{i} a_{i}+\tau
$$

where $\tau$ acts as a nonconservative source of parcel age. Mean ages were found to have a similar spatial pattern as found by using a "standard" technique of age inference (e.g. Broecker et al. [1991]), but were 50-200 years older. These deviations were attributed to adjustments to accommodate a steady-state solution and radiocarbon-age bias in observations due to ocean mixing.

Radiocarbon measurements also permit calculation of the transient time distributions (TTDs) (a form of Green functions) through the time rate of change of Equation 2.47 for a radioactively decaying tracer,

$$
\frac{d C}{d t}=\sum_{i=1}^{6} F_{i} C_{i}-F_{o} C-\lambda C=0
$$

(GH12, Eq. 8) where $F_{i}$ is volume flux from neighboring grid boxes, $F_{o}$ is the total volume flux into the neighboring boxes, and $\lambda$ is the radioactive decay rate. Mean- 
to-width ratios of the North Pacific TTDs provides a measure of the ratio of diffusive and advective processes in a flow configuration; by this metric, GH12 was found to be somewhat less diffusive than derived in a study using a GCM [Peacock and Maltrud, 2006], though both are consistent with an observational estimate Holzer and Primeau [2010].

Sources of uncertainty in GH12 include errors in surface radiocarbon values (reservoir ages), the presence of "exotic" unresolved water masses with extremely low ${ }^{14} \mathrm{C}$ ages, and errors in removing the influence of bomb radiocarbon. The Green functions derived by GH12 provide the modern mass circulation estimate used in Chapter 4. Future work should evaluate the IBC problem using other estimates of the modern circulation to estimate sensitivity. 


\section{Chapter 3}

\section{Benthic records of $\delta^{18} \mathbf{O}_{c c}$}

Sediment core records of $\delta^{18} \mathrm{O}_{c c}$ have been used for decades to investigate a variety of paleoceanographic phenomenon. This chapter has three parts. First, a brief overview of sediment core records is presented, with an emphasis on their error characteristics. The next section describes the eight sediment core records used in the deglacial tracer inverse problem discussed in Chapter 4. Finally, an error model is constructed for the models and an objective mapping scheme implemented in order to interpolate them in time and estimate the error covariance matrix.

\subsection{Records of $\delta^{18} \mathrm{O}_{c c}$}

The normalized ratio of stable oxygen isotopic compositions is defined as

$$
\delta^{18} \mathrm{O} \equiv\left[\frac{\left(\mathrm{O}^{18} / \mathrm{O}^{16}\right)_{\text {sample }}}{\left(\mathrm{O}^{18} / \mathrm{O}^{16}\right)_{\text {standard }}}-1\right] * 1000 \%
$$

where the "standard" is typically Pee Dee Belemnite (PDB) for carbonates and Vienna Standard Mean Ocean Water (VSMOW) for water and ice. The $\delta^{18} \mathrm{O}$ of seawater will 
be denoted $\delta^{18} \mathrm{O}_{s w}$ and the $\delta^{18} \mathrm{O}$ of calcium carbonate $\delta^{18} \mathrm{O}_{c c}$. As discussed in Section $(1.2), \delta^{18} \mathrm{O}$ has been used for decades in paleoceanography as an indicator of global ice volume and as an ocean water mass tracer [e.g. Imbrie et al., 1984].

Records of past $\delta^{18} \mathrm{O}_{s w}$ from the seafloor can be derived directly from interstitial pore waters in sediment mud and indirectly by analyzing the calcium carbonate shells of benthic foraminifera, which are deep-dwelling, single-celled protists. Empirical studies have shown that $\delta^{18} \mathrm{O}_{c c}$ is a function of both the $\delta^{18} \mathrm{O}_{s w}$ that bathed the foraminifer in situ at the time of calcification and the in situ temperature [Bemis et al., 1998]. In this study, $\delta^{18} \mathrm{O}_{c c}$ is treated as a passive, conservative tracer, meaning that it is assumed to have no sources or sinks away from the air-sea boundary and that its concentration does not affect the flow parameters. This approximation is discussed in greater detail in Chapter 4.

In order to obtain oxygen isotope records from sediment cores, a scoop of mud corresponding to a depth interval is taken. The sample is rinsed and sorted by size fraction to yield a collection of foraminiferal shells and other material; subsequently an investigator extracts particular species by hand under a microscope. The samples are then ground, homogenized, and analyzed in a mass spectrometer.

A sediment core record is a noisy, discrete subsample of a time-continuous physical process $s(t)$, such as the $\delta^{18} \mathrm{O}_{c c}$ at a sediment core site. There are numerous sources of noise in a sediment core record. Organisms may alias certain frequencies or features in $s(t)$ as they are calcifying, biasing results. During times of low sedimentation rates or low foraminiferal abundance, fewer organisms are available to sample and aggregate measurements will be less robust. Analytical procedures (selection of shells, cleaning, preparatory chemistry, etc.) can vary between sediment cores and sometimes within measurements. Calibration of calcite $\delta^{18} \mathrm{O}$ can vary between instruments by up to $0.3 \%$ [Ostermann and Curry, 2000]. The calibration of 
offsets between different species has an associated uncertainty that could introduce biases between monospecific records and errors within cores where multiple species are processed [e.g. Shackleton et al., 2000]. A host of diagenetic effects (chemical and bioturbative) can modify the record after deposition.

\section{Core chronologies}

Even in a case where independent age measurements are available for each data point, age model construction is non-trivial. ${ }^{14} \mathrm{C}$ is a cosmogenic nuclide that is generated in the atmosphere by the collision of cosmic rays with atmospheric nuclei (and by nuclear testing); this process is not constant, and the atmospheric concentration of ${ }^{14} \mathrm{C}$ varies in time correspondingly [Ramsey et al., 2012]. Radiocarbon projection ages, which are the sample age assuming constant ${ }^{14} \mathrm{C}$ at modern levels, are converted to calendar ages by projecting the age probability distribution onto a calibration curve. Even in the case when the only source of uncertainty in radiocarbon ages is believed to be from analytical uncertainty (and is assumed to be Gaussian), this procedure yields sample ages estimates that can be strongly non-Gaussian. Post-depositional effects such as bioturbation, in-situ ("diagenetic") modification of chemical properties, and distortion from core retrieval can all distort the timing of measurements further.

Because sedimentation rates vary with time, an age model (that is, a calendaryear assignment to each measurement) must be constructed independently for each sediment core. A typical procedure is to radiocarbon date the more abundant planktonic foraminifera from a subset of the sediment scoops and to interpolate converted calendar ages with depth in the intervening sediment.

Because radiocarbon is introduced into marine environments via air-sea gas exchange, so-called reservoir corrections must be made to account for the apparent ${ }^{14} \mathrm{C}$ age difference between the surface water and the atmosphere. For instance, the ra- 
diocarbon date of a modern planktonic foraminifera would typically be 400 before present (the nominal average reservoir age of the modern surface ocean) because that is the "age" of the typical in situ water mass derived using ${ }^{14} \mathrm{C}$. Changes in reservoir ages (especially at high latitudes) due to different circulation regimes have been implicated on deglacial time scales [Skinner and Shackleton, 2004] and potentially complicate conclusions about phase relationships between cores. Future work should consider reservoir changes in a more quantitative way. The reservoir offsets for the records in this study are the same as those used by previous authors.

\subsection{Descriptions of sediment core records}

This study considers eight sediment core records of $\delta^{18} \mathrm{O}_{c c}$ spanning the Last Deglaciation. The records were selected on the basis of sampling interval so as to resolve sub-millennial variability and on the basis of availability after a thorough but not exhaustive search. No comprehensive list of deglacial benthic $\delta^{18} \mathrm{O}$ records exists, and the data may be biased towards those records that are most cited or visible in the literature.

Figure 3.1 shows the distribution of the core locations. For convenience, records will be referred to by abbreviations denoting depth and their basin of origin rather than their cruise number; see Table 4.1. All of the records but SA3770 come from continental margins. Four of the records come from the Atlantic Ocean, two from the Indian Ocean, and one from the Pacific. Depths range from 1299m to 3770m.

The records are plotted in Figure 3.2. All records show a deglacial transition of $1-2 \%$ over the interval from 25 to $5 \mathrm{kyr}$, though the timing of the transition is different for different records, and higher-frequency variability is visible as well. The transi- 


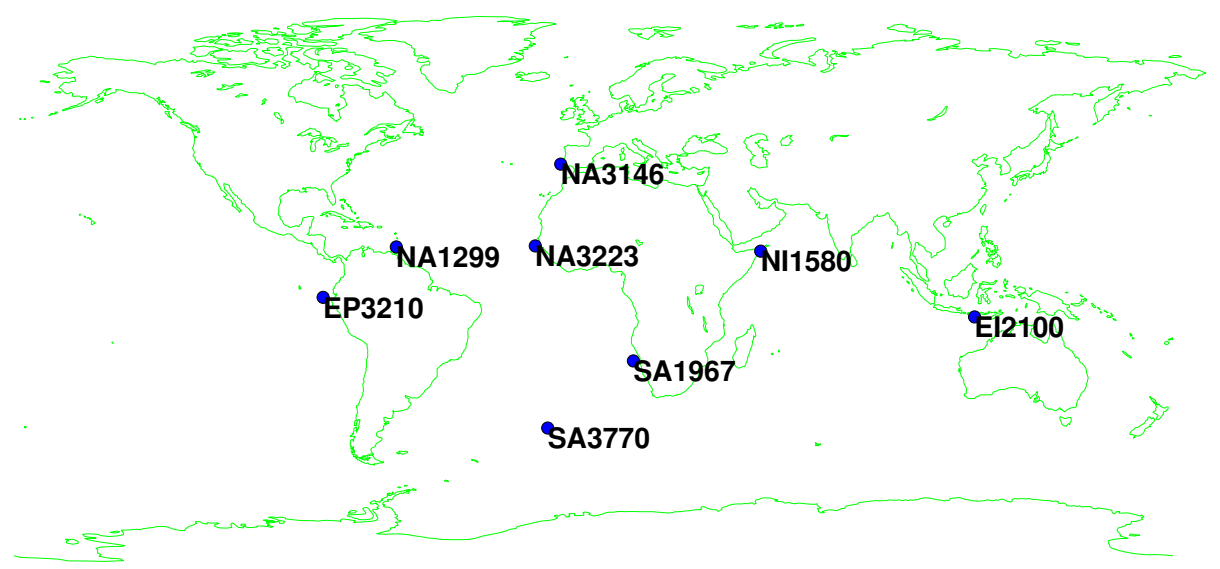

Figure 3.1: Locations for the eight sediment core records of $\delta^{18} \mathrm{O}$ used in this study. Labels are abbreviations based on ocean basin and water depth; the original core names are found in Table (3.1).

tion in EP3210 (one of the two cores that was discussed in Skinner and Shackleton [2005], Wunsch and Heimbach [2008], and Gebbie [2012]; the other is NA3146) appears markedly later than in other records. The shallower records (NI1580, EI2100, SA1967, and NA1299) appear to share a two-stage decrease in $\delta^{18} \mathrm{O}$, with the first transition occurring between about 18 and 16kya. All of the records span the interval from $25 \mathrm{kyr}$ to $5 \mathrm{kyr}$ except SA1967, which begins slightly after $24 \mathrm{kyr}$. The records are unevenly spaced in time, and in several cases there are gaps of nearly 1000 years.

The following section summarizes the development of each record and age model. A list of references is included in Table (3.1). Analytical procedures vary between cores. Most of the records use tests from the genus Cibicidoides, but NA3146 and EP3210 use other genera. All records are corrected to the Uvigerina standard for $\delta^{18} \mathrm{O}_{c c}$; e.g., Cibicidoides foraminifera are offset by the conventional $0.64 \%$. In the case where multiple sample values exist contemporaneously in a record, the average value was used.

\section{GeoB1711 (SA1967)}

Gravity core GeoB1711 (referred to in plots as SA1967) was retrieved south of the 
SA1967

C. wuellerstorfi

NA3223

C. wuellerstorfi

NA1299

Cibicides

SA3770

C. kullenbergi

El2100

Cibicides

NA3146

G. affinis

P. wuellerstorfi

NI1580

Cibicides

EP3210

uvi

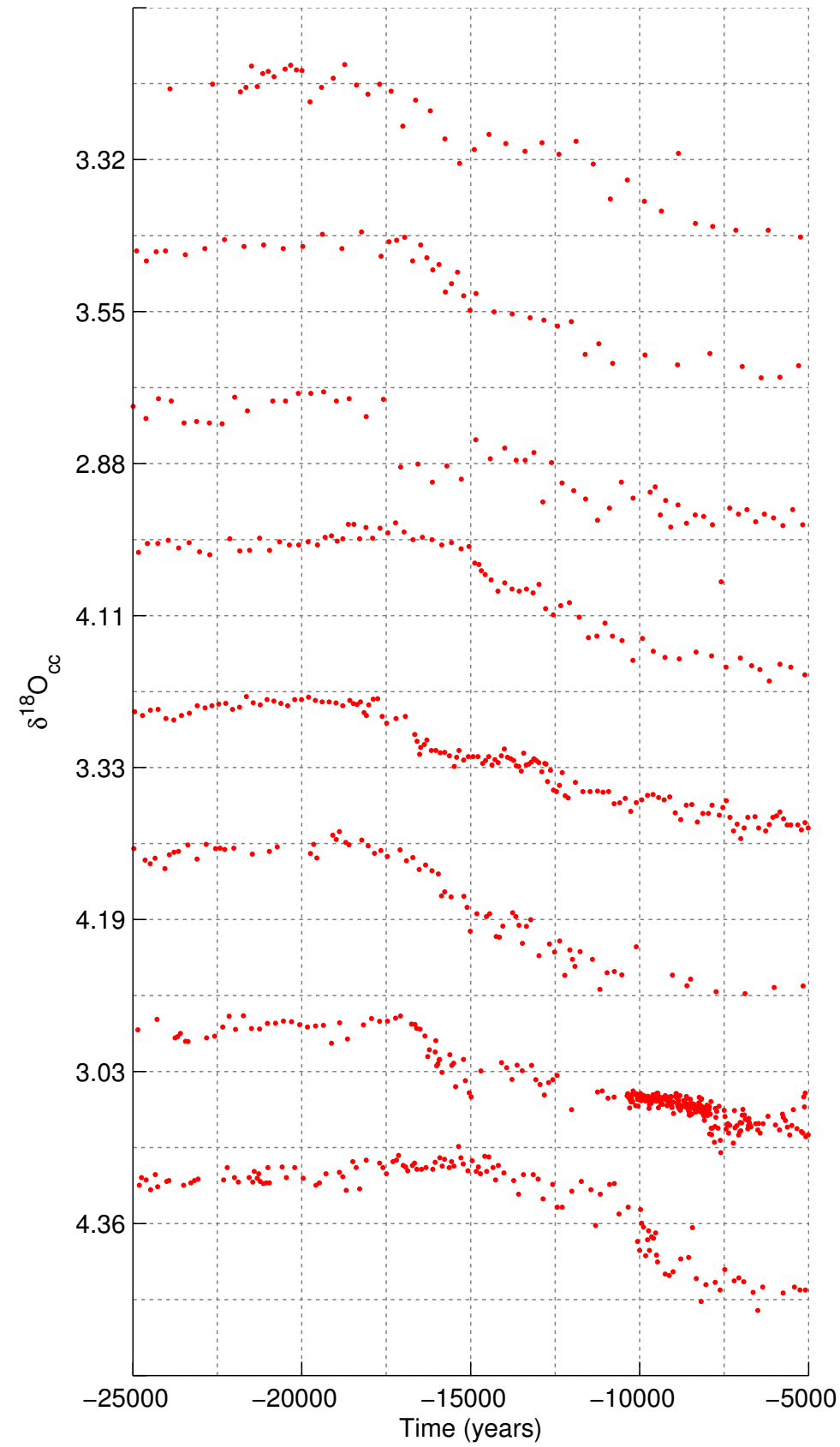

Figure 3.2: The eight benthic foraminiferal $\delta^{18} \mathrm{O}$ records used in this work plotted about their means. Computation of record mean values is discussed in section 3.3.2. Grid spacing in the vertical is $1 \%$ and in the horizontal is 1000 years. Some records contain measurements from multiple benthic foraminferal species. 


\begin{tabular}{lccccc}
\hline & & & & & \multicolumn{2}{c}{ References } \\
\cline { 5 - 6 } Site & Ocean & Depth & Name & Benthic $\delta^{18} \mathrm{O}$ & Dating \\
\hline GeoB1711 & Atl S & 1967m & SA1967 & Little et al. [1997a] & Vidal et al. [1999] \\
GeoB9526-4 & Atl N & $3223 \mathrm{~m}$ & NA3223 & Zarriess and Mackensen [2011] & Zarriess and Mackensen [2010] \\
M35003-4 & Atl N & $1299 \mathrm{~m}$ & NA1299 & Hüls and Zahn [2000] & Rühlemann et al. [1999] \\
MD07-3076Q & Atl S & $3770 \mathrm{~m}$ & SA3770 & Waelbroeck et al. [2011] & Skinner et al. [2010] \\
MD98-2165 & Ind E & $2100 \mathrm{~m}$ & EI2100 & Waelbroeck et al. [2006] & Waelbroeck et al. [2006] \\
MD99-2334K & Atl N & $3146 \mathrm{~m}$ & NA3146 & Skinner and Shackleton [2004] & Skinner et al. [2003] \\
NIOP-905 & Ind N & $1580 \mathrm{~m}$ & NI1580 & Jung et al. [2001] & Ivanochko et al. [2005] \\
& & & & Jung et al. [2009] & Skinner and Shackleton [2005] \\
\hline
\end{tabular}

Table 3.1: Summary of benthic foraminferal $\delta^{18} \mathrm{O}$ records. The "Name" field indicates reference codes used in this thesis for ease of discussion. SA1967 refers to a South Atlantic core at $1967 \mathrm{~m}$ depth, NI1580 to a North Indian core at $1580 \mathrm{~m}$ depth, etc.

Walvis Ridge at $23^{\circ} 18.9 \mathrm{~S}, 12^{\circ} 22.6 \mathrm{E}$ at a depth of 1967 meters by Meteor cruise M20/2 in 1991. The core was $1066 \mathrm{~cm}$ long and was sampled at $5 \mathrm{~cm}$ intervals. Tests of Globorotalia inflata ( $G$. inflata, planktonic) and Cibicidoides wuellerstorfi Schwager (C. wuellerstorfi, benthic) were picked from the wet-sieved 250-500 $\mu \mathrm{m}$ fraction to yield 8-10 well-preserved, clean specimens from each sample. $\delta^{18} \mathrm{O}_{c c}$ analyses were conducted on benthic specimens at Kiel University using a Finnigan MAT 251 mass spectrometer with analytical precision $\pm 0.08 \%$ and calibrated to the PDB standard [Little et al., 1997a].

In Little et al. [1997a], the age model for GeoB1711 was constructed by maximizing the correlation between the benthic $\delta^{18} \mathrm{O}_{c c}$ record and the stacked record of Imbrie et al. [1984]. Additional age constraints were developed by Little et al. [1997b], who obtained 4 AMS ${ }^{14} \mathrm{C}$ dates on $G$. inflata, and by Vidal et al. [1999], who ${ }^{14} \mathrm{C}$-dated planktonic foraminifera $G$. inflata and $G$. bulloides in five IRD layers corresponding 
to Heinrich events. One of the dates published by Little et al. [1997b] implies an exceptionally high sedimentation rate during a 1000-year interval and is discarded in Vidal et al. [1999] and in this study. All ages have a 400-year reservoir correction applied. Dates were converted from ${ }^{14} \mathrm{C}$ years to calendar years using OxCal 4.1 [Bronk Ramsey, 2009, Reimer et al., 2009] and calendar ages were linearly interpolated with sediment core depth between ${ }^{14} \mathrm{C}$ tie points.

\section{GeoB9526-5 (NA3223)}

Gravity core GeoB9526-5 (referred to in plots as NA3223) was recovered off of West Africa at $12^{\circ} 26.1^{\prime} \mathrm{N}, 18^{\circ} 03.4^{\prime} \mathrm{W}$ from a depth of 3231 meters. The core was 1080 $\mathrm{cm}$ long and was sampled at $5 \mathrm{~cm}$ intervals. Between 2 and 5 tests of $C$. wuellerstorfi were extracted from the $>125 \mu \mathrm{m}$ fraction of each sample. Measurements of $\delta^{18} \mathrm{O}_{c c}$ were performed using a Finnigan MAT 151 mass spectrometer at Kiel University with analytical uncertainty of $\pm 0.08 \%$ and standardized to Vienna PDB [Zarriess and Mackensen, 2011].

The age model is constrained by seven ${ }^{14} \mathrm{C}$ AMS dates calculated using multispecies samples of shallow-dwelling species from the $>125 \mu \mathrm{m}$ fraction at the Leibniz Laboratory for Radiometric Dating and Stable Isotope Research Kiel, Germany [Zarriess and Mackensen, 2010]. These authors applied a 400-year reservoir correction and used the approach of Fairbanks et al. [2005] to convert from ${ }^{14} \mathrm{C}$ years to calendar years. Additional age constraints were provided in Zarriess and Mackensen [2010] by aligning the planktonic $\delta^{18} \mathrm{O}_{c c}$ record with that of MD95-2042.

\section{M35003-4 (NA1299)}

Core M35003-4 was raised at the southern end of the Lesser Antilles island chain in the Caribbean Sea $\left(12^{\circ} 5.4^{\prime} \mathrm{N}, 61^{\circ} 14.6^{\prime} \mathrm{W}\right)$ from a depth of 1299 meters. Three to five 
benthic tests were picked from the $>250 \mu \mathrm{m}$ fraction for $\delta^{18} \mathrm{O}_{c c}$ analysis. Epibenthic species C. wuellerstorfi, C. kullenbergi, and C. pseudoungerianus were used where available; below $450 \mathrm{~cm}$ in the core (roughly $25 \mathrm{kya}$ ), the endobenthic species Uvigerina peregrina was used due to low epibenthic species abundances. Measurements of $\delta^{18} \mathrm{O}_{c c}$ were performed using a Finnigan MAT 252 mass spectrometer at Kiel University with analytical uncertainty of $\pm 0.066 \%$ and standardized to Vienna PDB [Zahn and Stüber, 2002].

A total of twenty ${ }^{14} \mathrm{C}$ AMS dates are available to constrain the core chronology. Twelve dates were run on samples of G. ruber (white) [Hüls and Zahn, 2000] and eight were run on a mixture of G. ruber (white) and G. sacculifer [Rühlemann et al., 1999]. All dates were obtained at the Leibniz Laboratory in Kiel. A 400 year reservoir correction was applied to the ${ }^{14} \mathrm{C}$ data, which I then converted to calendar ages using OxCal and interpolated linearly with depth to construct age estimates for each measurement. "Fine-tuning" of the record chronology via comparison with the GISP2 $\delta^{18} \mathrm{O}$ record as performed in Hüls and Zahn [2000] is not used here.

\section{MD07-3076Q (SA3770)}

Core MD07-3076Q was raised in the Southern Ocean on the eastern flank of the Mid-Atlantic Ridge $\left(44^{\circ} 09.19^{\prime} \mathrm{S}, 14^{\circ} 13.70^{\prime} \mathrm{W}\right)$ at a depth of 3770 meters. Samples were taken at $2 \mathrm{~cm}$ intervals and benthic foraminifera C. kullenbergi were picked from the $>150 \mu \mathrm{m}$ fraction for $\delta^{18} \mathrm{O}_{c c}$ analysis. Samples were run on a Finnigan $\Delta+$ mass spectrometer at LSCE (Gif-sur-Yvette) and calibrated to the VPDB standard, with reproducibility $1 \sigma$ of $\pm 0.05 \%$.

The chronology for MD07-3076 is based on monospecific planktonic foraminiferal ${ }^{14} \mathrm{C}$ ages taken from 59 samples along the core. Corrections for variable reservoir age

effects were applied by 1) correlating temperature trends as measured by Antarctic 
ice-core records and SSTs as measured by planktonic foraminifera and 2) comparing reservoir corrections to those published for core TNO57-21 in the Cape Basin [Skinner et al., 2010]. These corrections are used in this study.

\section{MD98-2165 (EI2100)}

Core MD98-2165 was recovered on board the Marion Dufresne in the eastern tropical Indian Ocean $\left(9^{\circ} 38.96^{\prime} \mathrm{S}, 118^{\circ} 20.31^{\prime} \mathrm{E}\right)$ at $2100 \mathrm{~m}$ depth. Samples of $1 \mathrm{~cm}$ were taken every 2 to $4 \mathrm{~cm}$. Cibicides were picked in the $>150 \mu \mathrm{m}$ fraction, with a preference for C. wuellerstorfi, though in some levels Cibicides spp. (C. ungerianus, C. pachyderma, C. kullenbergi) were analyzed. $\delta^{18} \mathrm{O}_{c c}$ analyses were performed at LSCE on a Finnigan MAT251 mass spectrometers, with reproducibility $1 \sigma$ of $\pm 0.05 \%$. No offsets in isotopic values were observed between $C$. wuellerstorfi and the other species [Waelbroeck et al., 2006].

The age model was constructed [Waelbroeck et al., 2006] using 20 AMS ${ }^{14} \mathrm{C}$ dates measured on G. ruber white samples. Carbon ages were converted to calendar ages using Calib 4.3 [Stuiver and Braziunas, 1993] and the method of Bard et al. [1998] after subtracting a 400 year reservoir correction. Two ages near the top of the core gave anomalously old ages and were discarded. Sample ages were linearly interpolated with depth between ${ }^{14} \mathrm{C}$ control points.

\section{MD99-2334K (NA3146)}

Core MD99-2334K was recovered from the Iberian Margin $\left(37^{\circ} 48^{\prime} \mathrm{N}, 10^{\circ} 10^{\prime} \mathrm{W}\right)$ at a depth of $3146 \mathrm{~m}$. Several sets of measurements and accompanying chronologies are available for this record [Skinner et al., 2003, Skinner and Shackleton, 2004, 2005]; here the record from Skinner and Shackleton [2005] is used because of its high temporal resolution. Benthic foraminifera P. wuellerstorfi and G. affinis were picked from 
the $>212 \mu \mathrm{m}$ fraction of sediment samples. On average 3 P. wuellerstorfi and 20 G. affinis were picked from each sample; the former was used strictly for stable isotope measurements and the latter for both stable isotope and $\mathrm{Mg} / \mathrm{Ca}$ measurements. Oxygen isotope measurements were performed on a Micromass Multicarb Sample Preparation system attached to a PRISM mass spectrometer. Measurements were standardized using VPDB and had an analytical precision better than $0.08 \%$.

The age model is based upon synchronization of the planktonic $\delta^{18} \mathrm{O}_{c c}$ record with the GISP2 ice core record. This synchronization is corroborated using $17{ }^{14} \mathrm{C}$ dates calculated at LSCE, Gif-sur-Yvette on monospecific samples of G. bulloides and N. pachyderma. A 400-year reservoir age is used [Skinner et al., 2003].

\section{NIOP-905 (NI1580)}

Piston core NIOP-905 was recovered off of the continental slope of Somalia $\left(10^{\circ} 46^{\prime}\right.$ $\left.\mathrm{N}, 51^{\circ} 57^{\prime} \mathrm{E}\right)$ at a depth of $1580 \mathrm{~m}$. Oxygen isotope measurements were made on foraminifera picked from the $250-350 \mu \mathrm{m}$ fraction. Values are the average of multiple analyses of C. kullenbergi tests; in some cases in the older (>11 kya) parts of the core, Cibicidoides spp. are used to avoid record gaps where kullenbergi is sparse. The internal reproducibility for these measurements is $\pm 0.05 \%$ [Jung et al., 2009, 2001]. The age model is based upon twelve ${ }^{14} \mathrm{C}$ dates of samples from the past 31 kya [Jung et al., 2001].

\section{TR163-31b (EP3210)}

Kasten core TR163-31b was recovered in the eastern equatorial Pacific ( $3^{\circ} 37.2^{\prime}$ $\left.\mathrm{S}, 83^{\circ} 58^{\prime} \mathrm{W}\right)$ at $3210 \mathrm{~m}$. Benthic foraminifera Uvigerina senticosa and C. wuellerstorfi were picked from the $>250 \mu \mathrm{m}$ fraction at roughly $2 \mathrm{~cm}$ intervals. Analytical measurement noise is roughly $\pm 0.07 \%$ [Shackleton et al., 1988]. 
The age model for TR163-31b was constructed from $19{ }^{14} \mathrm{C}$ measurements performed on planktonic dutertrei [Shackleton et al., 1988] and calibrated [Skinner and Shackleton, 2005] to calendar ages using the datasets of Bard et al. [1998] and Hughen et al. [2000, 2004]. A 580-year reservoir age correction was subtracted, reflecting the modern radiocarbon age in the near-surface ocean above this core site.

\subsection{Error models, mean estimation, and objective mapping}

Time series of geochemical measurements sampled from sediment cores are unevenly spaced with sources of error from numerous physical, biological, and chemical processes. In order to use sediment core records in an inverse context, it is necessary to have an explicit statement of the uncertainty of each of the measurements. Such statements are not always reported in published accounts of recovered records; instead, a typical value for the analytical uncertainty or reproducibility error is given for the entire record. This section develops and applies an error model to the eight sediment cores used in the study that incorporates information about sample spacing and a conservative uncertainty estimate for each measurement.

\subsubsection{The sediment core error model}

Construction of the error model occurs in three steps. First, each record $\mathbf{y}$ is separated into a "mean" and a "fluctuating" component,

$$
\mathbf{y}=\mathbf{d} m+\mathbf{f}
$$


where $\mathbf{y}$ is an $M$-vector of observations, $m$ is a scalar mean value, $\mathbf{f}$ is the $M$-vector corresponding to the fluctuating part, and $\mathbf{d}$ is an $M$-vector of ones. These components represent the entire observed signal $\mathbf{y}$, including the observational error $\mathbf{n}$. The procedure for finding the mean is outlined below and described in greater detail in Wunsch [2006]. Because some (potentially large) sources of error affect only the record means, the mean and fluctuating parts are isolated in the inverse problems posed in Chapter 4. Next, a "conservative" estimate of $0.2 \%$ (pers. comms., E. Boyle and L. Keigwin) uncertainty is assumed for every $\delta^{18} \mathrm{O}_{c c}$ measurement. Finally, each record is interpolated to be uniformly spaced in time with a sampling interval of 200 years, reflecting a rough average sampling rate (though this varies between and within cores).

The interpolation of the records addresses a complication arising from the use of unevenly-spaced records in a tracer inverse problem. In an unevenly sampled record, some time intervals are more densely sampled than others, with the result that the minimization of the cost function preferentially reduces model-data misfit at some intervals in time. To enforce some parity of influence among different intervals without imposing solution priors, objective mapping is used to relate the unevenly spaced record to a regularly spaced grid.

The statistical treatment of time uncertainty is an emerging discipline [Blaauw et al., 2003, Huybers and Wunsch, 2004, Buck and Millard, 2004, Buck, 2004, Haam and Huybers, 2010, Tierney and Anchukaitis, 2011] and no additional steps were taken to account for uncertainty due to age models beyond the steps taken in generating the original records.

Implementing a more sophisticated error model for sediment cores may be an important step in future paleoceanographic inverse problems. A procedure that used sampling interval length and species abundance was considered but rejected in the 
current approach because it would have required substantial testing and validation. Particular care should be given to records that rely upon different species at different times.

\subsubsection{Mean estimation and objective mapping}

This section outlines procedures for isolating the means of records and interpolating them to uniform spacing in time. Much of the material is a recapitulation of standard methods.

\section{Estimation of the record mean}

The problem of estimating the mean of a vector of observations can be written [Wunsch, 2006, Eqn. 2.412]

$$
\begin{aligned}
\mathbf{d} m+\mathbf{f} & =\mathbf{y} \\
\mathbf{d} & =[1,1, \ldots 1]^{\top} .
\end{aligned}
$$

When no prior distribution is specified for $m$, the minimum variance solution $\tilde{m}$ to (3.1) (that is, the solution that minimizes the variance of the solution about the true value $m$ ) is the estimate that minimizes the cost function [Wunsch, 2006, p. 130]

$$
J(m)=(\mathbf{y}-\mathbf{d} m)^{\top} \mathbf{R}_{\mathrm{ff}}^{-1}(\mathbf{y}-\mathbf{d} m)=\mathbf{f}^{\top} \mathbf{R}_{\mathrm{ff}}^{-1} \mathbf{f} .
$$

When the fluctuating part of the record is stationary white noise, $\mathbf{R}_{\mathrm{ff}}^{-1}=\mathbf{I} \sigma^{2}$, where

I is the identity matrix and $\sigma^{2}$ is the variance of the white noise. Then $\tilde{m}$ and its 
uncertainty $P$ are given by the familiar equations

$$
\begin{aligned}
\tilde{m} & =\frac{1}{M} \sum_{i=1}^{M} y_{i} \\
P_{m} & =\sigma^{2} / M .
\end{aligned}
$$

The approximation that climate time series are spectrally white is a poor one; physical evidence [Frankignoul and Hasselmann, 1977, Huybers and Curry, 2006] suggests that climate spectra are red over large frequency ranges. The more general solution to (3.1) is given by

$$
\begin{aligned}
\tilde{m} & =\left\{\mathbf{d}^{\top} \mathbf{R}_{\mathrm{ff}}^{-1} \mathbf{d}\right\}^{-1} \mathbf{d}^{\top} \mathbf{R}_{\mathrm{ff}}^{-1} \mathbf{y} \\
\tilde{\mathbf{f}} & =\mathbf{y}-\mathbf{d} \tilde{m} \\
P_{m} & =\left\{\mathbf{d}^{\top} \mathbf{R}_{\mathrm{ff}}^{-1} \mathbf{d}\right\}^{-1},
\end{aligned}
$$

a form of the standard weighted least-squares solution (2.6). The mean and its uncertainty can take dramatically different values from the "standard" form in (3.2) depending on the covariance of individual observations [Wunsch, 2006].

\section{One-dimensional objective mapping}

Begin with the estimated fluctuating part of the record $\tilde{\mathbf{f}}$ defined in Equation (3.3); removing the least-squares mean estimate simplifies notation and makes the interpolation unbiased [Rybicki and Press, 1992]. For consistency of notation, this fluctuating part will be written without a tilde in this section, $\tilde{\mathbf{f}} \rightarrow \mathbf{f}$.

The tracer inverse problem for the fluctuating part of a single sediment core record may be written

$$
\mathbf{f}=\mathbf{E q}+\mathbf{n}
$$


where $\mathbf{E}(M \times N)$ is one of the matrices constructed from Green functions that is discussed in Chapter 2, $\mathbf{q}$ is an $N$-vector of controls, and $\mathbf{n}$ is an $M$-vector of noise. Equation (3.4) may also be thought of as a decomposition of $\mathbf{f}$ into a "signal" component $\mathbf{s} \equiv \mathbf{E q}$ and the noise component $\mathbf{n}$,

$$
\mathbf{f}=\mathbf{s}+\mathbf{n}
$$

Each of the three vectors $\mathbf{f}, \mathbf{s}$, and $\mathbf{n}$ is a series of realizations of time-continuous random processes $f(t), s(t)$, and $n(t)$ at times $t=t_{1}, t_{2}, \ldots t_{M}$. In the objective mapping problem, the goal is to estimate a value of the function $s(t)$ at any time $t^{*}$. An estimate of $s\left(t^{*}\right)$ that is linear in the observations $\mathbf{f}=\left[f_{1}, f_{2}, \ldots f_{M}\right]$ can be written

$$
s\left(t^{*}\right)=\sum_{i=1}^{M} e_{i}^{*} f_{i}+u^{*},
$$

where the $e_{i}^{*}$ are coefficients and $u^{*}$ is the discrepancy.

Define the covariance function of the mean-removed, time-continuous process $s(t)$ to be

$$
R_{s s}\left(t, t^{*}\right)=\left\langle s(t) s\left(t^{*}\right)\right\rangle .
$$

Assuming that the signal and noise are uncorrelated, the minimum variance estimate of $s\left(t^{*}\right)$, which minimizes the objective function $J\left(e_{i}^{*}\right)=\left(u^{*}\right)^{2}$, is [Wunsch, 2006, $3.27]$

$$
\begin{aligned}
\mathbf{s}\left(t^{*}\right) & =\tilde{\mathbf{e}^{*} \mathbf{f}} \\
\tilde{e}_{i}^{*} & =\sum_{j=1}^{M} R_{s s}\left(s\left(t_{j}\right) s\left(t^{*}\right)\right)\left[\mathbf{R}_{\mathbf{s s}}+\mathbf{R}_{\mathbf{n n}}\right]_{j, i}^{-1}
\end{aligned}
$$


where $\mathbf{R}_{\mathrm{ss}}$ and $\mathbf{R}_{\mathbf{n n}}$ are the covariances of the zero-mean signal and noise process at the observed times and $\left[\mathbf{R}_{\mathbf{s s}}+\mathbf{R}_{\mathbf{n n}}\right]_{j, i}^{-1}$ denotes the $j, i^{\text {th }}$ element of the inverse matrix. The minimum possible uncertainty (defined in terms of estimates of $s(t)$ at two times $t_{\alpha}^{*}$ and $t_{\beta}^{*}$ ) is [Wunsch, 2006, 3.28]

$$
\mathbf{P}\left(t_{\alpha}^{*}, t_{\beta}^{*}\right)=R_{s s}\left(t_{\alpha}^{*}, t_{\beta}^{*}\right)-\sum \sum R_{s s}\left(t_{\alpha}^{*}, t_{j}\right)\left[\mathbf{R}_{\mathbf{s s}}+\mathbf{R}_{\mathbf{n n}}\right]_{j, i}^{-1} R_{s s}\left(t_{i}, t_{\beta}^{*}\right) .
$$

\section{Estimation of the signal covariance function $R_{s s}(\cdot, \cdot)$}

In order to apply the results derived in 3.3.2 to the benthic $\delta^{18} \mathrm{O}_{c c}$ records, it is necessary to estimate the signal covariance at both the $t_{i}$ (the times of observation in the original record) and the $t_{i}^{*}$ (the new times when we would like to estimate $s(t)$ ) as well as the noise covariance matrix $\mathbf{R}_{\mathbf{n n}}$. The noise covariance matrix is assumed $a$ priori to be $\mathbf{R}_{\mathbf{n n}}=\mathbf{I} \sigma^{2}$, where $\sigma=0.2 \%$ and $\mathbf{I}$ is the identity matrix. The procedure used to estimate $R_{s s}$ is based on an application to astrophysical data described in Press et al. [1992] and Rybicki and Press [1992].

First assume that the process $s(t)$ is stationary so that

$$
R_{s s}\left(t, t^{*}\right)=R\left(t-t^{*}\right) \equiv R(\tau)
$$

where $R(\tau)$ is the autocovariance function for a lag $\tau$. The so-called structure function or variogram $V(\tau)$ is closely related to the autocovariance,

$$
\begin{aligned}
V(\tau) \equiv \frac{1}{2}\left\langle[s(t+\tau)-s(t)]^{2}\right\rangle & =\left\langle s^{2}(t)\right\rangle-\langle s(t+\tau) s(t)\rangle \\
& =\left\langle s^{2}(t)\right\rangle-R(\tau)
\end{aligned}
$$


and is readily estimated from the data. To estimate the structure function, the lags $\tau_{i j} \equiv\left|t_{i}-t_{j}\right|$ and estimates of the structure function $v_{i j} \equiv\left(f_{i}-f_{j}\right)^{2}-n_{i}^{2}-n_{j}^{2}$ were computed for each pair of points within each sediment core record. A power-law estimate of $V(\tau)$ was computed by fitting a line to binned and averaged point-wise estimates of the structure function in log-log space. The signal variance $\left\langle s^{2}(t)\right\rangle$ was estimated using the sample variance $\left\langle\mathbf{f}^{\top} \mathbf{f}\right\rangle$ [Rybicki and Press, 1992, Press et al., 1992].

\subsubsection{Application to benthic $\delta^{18} \mathrm{O}_{c c}$ records}

The objective mapping of the sediment core records onto an evenly-spaced 200 year mesh spanning $25 \mathrm{kya}$ to $5 \mathrm{kya}$ is plotted in Figure 3.3. The interpolated function is smoother than the raw data. The uncertainty (shading) grows when estimates are far from observed values or when observations are in disagreement. Mean values for each record were also inferred, but their physical meaning is unclear because of the nonstationarity of the records. Treatment of the mean in the tracer inverse problem is elaborated upon in the next chapter.

The uncertainty plotted in Figure 3.3 is the square root of the diagonal of the $\mathbf{P}$ matrix defined in Equation (3.6). However, the $\mathbf{P}$ matrix is not diagonal. The interpolated estimates inherit their uncertainty from the observations, of which the estimates are linear combinations. Thus the error in interpolated estimates that are nearby in time is expected to covary. The full $\mathbf{P}$ matrix is used in Chapter 4 . 
SA1967

C. wuellerstorfi

NA3223

C. wuellerstorfi

NA1299

Cibicides

SA3770

C. kullenbergi

El2100

Cibicides

NA3146

G. affinis

P. wuellerstorfi

NI1580

Cibicides

EP3210

uvi

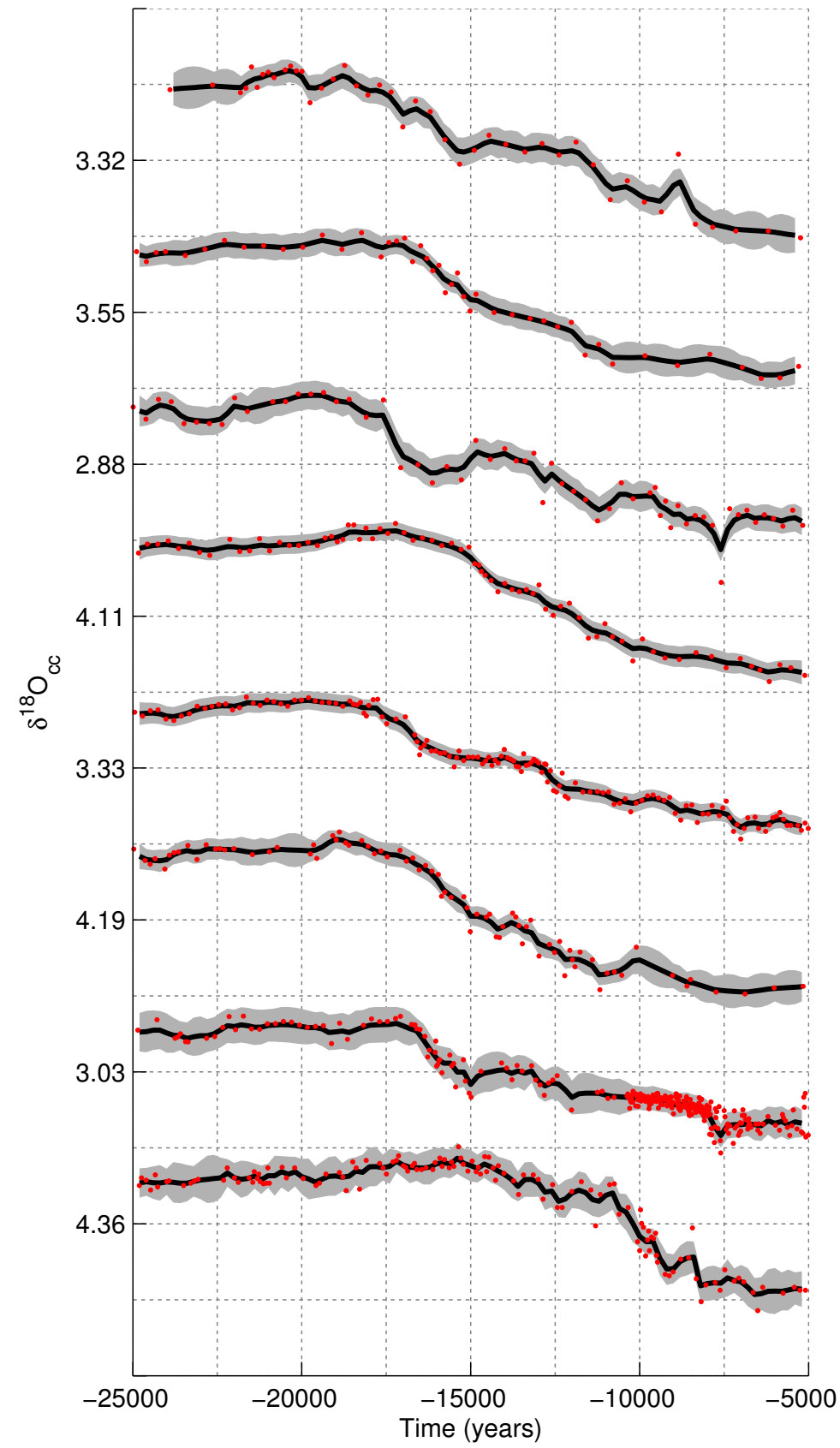

Figure 3.3: Similar to Figure 3.2, but including objectively-mapped samples (black lines) and their uncertainty (shading) every 200 years. Grid spacing in the vertical is $1 \%$ and in the horizontal is 1000 years. The plotted uncertainties are the square roots of the diagonal of the uncertainty matrix $\mathbf{P}$ (Eqn 3.6). 


\section{Chapter 4}

\section{The deglacial benthic $\delta^{18} \mathrm{O}$ tracer problem}

The distribution of tracers in the ocean is determined by boundary conditions imposed at the ocean surface and by the ocean circulation. It thus bears the imprint of both processes. As in Chapter 2, the problem of inferring boundary condition values under an assumed tracer transport model is called the inverse boundary condition (IBC) problem. This chapter solves the IBC problem in a discretized model representation of the modern ocean, using the ocean mass circulation estimate of Gebbie and Huybers [2012]. In this inverse problem, the boundary conditions or "controls" are (unknown) patterns of tracer concentration in the ocean mixed layer (ML) grid boxes, and the system state consists of the rest of the ocean. Deglacial sediment core records are treated as noisy observations of ocean tracer concentration and a solution to the IBC problem is sought using the tracer inverse approaches described in Chapter 2.

The scientific context for this chapter is outlined in Chapter 1. Previous work [Skinner and Shackleton, 2005, Waelbroeck et al., 2006, 2011] suggested that differ- 
ences between multiple contemporaneous records of benthic sediment core $\delta^{18} \mathrm{O}_{c c}$ at remote core sites were due to circulation changes. Seeking a solution to the IBC problem is a complementary approach to inferring circulation changes and asks whether the data can be explained assuming the a steady-state estimate of the modern circulation under changing boundary conditions (see Section 2.3, Gebbie [2012]; Wunsch [1987] is an analog). The guiding principle behind the IBC approach is Occam's ra-

zor: while it is well known that light $\delta^{18} \mathrm{O}_{c c}$ entered the ocean during the deglaciation because of melting ice sheets, the evidence for changes in ocean circulation during the deglaciation is indirect and inconclusive, and the physical mechanisms for such changes are not clear. If variability in boundary conditions alone suffices to explain the benthic records, then implicating changes in ocean circulation violates parsimony and is unnecessary.

A novel contribution of this work is to estimate the uncertainty of the IBC solution. This uncertainty has two components: one comes from noise in the observations, and one comes from the nullspace of the problem (see 2.2.2 for a discussion of the nullspace). Features that lie in the nullspace of the IBC problem are not constrained by the data, and additional information must be brought to bear in order to say something about these features.

\subsection{Assumptions}

Several assumptions and procedural choices are made in finding a solution to the IBC problem.

First, the approximation is made that $\delta^{18} \mathrm{O}_{c c}$ is a passive tracer, meaning that the tracer does not affect the flow field. The passive assumption runs counter to the expectation that meltwater input, which is expected to covary with $\delta^{18} \mathrm{O}_{c c}$ and has 
a dynamical effect due to its density anomaly compared to seawater, will alter the ambient ocean circulation in some way. Friedrich and Timmermann [2012] pursued an approach to the $\delta^{18} \mathrm{O}$ problem using an estimate of the ocean's sensitivity to freshwater forcing provided by a numerical model. However, this sensitivity is generally not well known; in particular, the result that meridional overturning shuts down subsequent to large coastal freshwater inputs (as seen in "hosing" experiments, e.g. Manabe and Stouffer [1988]) is subject to question [Condron and Winsor, 2011]. Simulations by Siberlin [2010] suggest that the passive approximation is not unreasonable for small $(\sim 0.001 \mathrm{~Sv})$ freshwater fluxes.

Second, the assumption is made that $\delta^{18} \mathrm{O}_{c c}$ is a conservative tracer, meaning that there are no interior sources or sinks of the tracer away from the surface boundaries. At least two factors are ignored in approximating $\delta^{18} \mathrm{O}_{c c}$ as a conservative tracer. First, the ratio of conserved quantities is itself not conserved, a fact that has been dismissed by other authors on the basis of the low abundance of ${ }^{18} \mathrm{O}$ [Dail, 2012]. Second, $\delta^{18} \mathrm{O}_{c c}$ is the function of two quantities, $\delta^{18} \mathrm{O}_{s w}$ and in-situ temperature $T$; while the former is conserved, the latter, due to compressive effects, is not. The nonconservative compressive effects correspond to a $0.02 \% 0-0.07 \%$ negative deviation in the time-mean $\delta^{18} \mathrm{O}_{c c}$ of each record depending on depth and latitude [Fofonoff and Millard [1983], Bryden [1973], Saunders [1981], assuming the linear paleotemperature equation of Bemis et al. [1998]]. This deviation is not treated explicitly, but it is expected to be removed as part of the record means.

Third, no solution priors are enforced by column weighting, which selects for solution properties that are a priori desirable (see Section 2.2.1 for a discussion of column weighting). Rather, the solution is controlled only through the definition of an effective nullspace by choice of the SVD truncation parameter $K^{\prime}$, which limits the solution variance and reduces the solution covariance (at the expense of increasing 
solution bias and uncertainty from nullspace contributions). Gebbie [2012] found a solution by minimizing a cost function that penalized deviations in the solution from a "first guess" pattern of deglacial surface $\delta^{18} \mathrm{O}_{s w}$ and $\theta$ evolution at every surface grid point; rapid transitions in time; and large horizontal tracer gradients. Reflecting data constraints, his posterior solution deviates from the modern prior in several highlatitude regions. In this thesis, solution features that cannot be resolved by the data, including most features of the modern tracer distribution, are set to zero (yielding the so-called "particular" SVD solution, Wunsch [2006]).

Fourth, the IBC problem is decomposed into three components - the time mean, a "fluctuating" or "mean-removed" problem, and the initial value problem of the fluctuating part - each of which is treated separately. The separation of the mean from the fluctuating part is motivated by properties of errors in sediment cores, wherein some sources of error, such as intercalibration error and species biases, affect mainly record means. It is also conventional wisdom to remove record means before performing regressions. The physical content of the mean values is uncertain because they depend strongly upon the phase of the low-frequency deglacial signal. This dependence dictates that the mean and fluctuating problems cannot be solved separately, and instead the record means are simply subtracted and only the fluctuating part treated. The separation of the initial condition problem makes the fluctuating problem more tractable and permits the evaluation of a steady tracer solution during the last glacial maximum (LGM), which directly preceded the deglaciation.

Additional assumptions are made about properties of the data (3.3.3), properties of the model (4.2.1), and the representation of the model state space by the data (4.2.2) that are discussed in greater detail in the respective sections. 


\subsection{Formulation of the inverse boundary problem}

Consider again the canonical statement (2.20) of the forward state vector evolution under boundary conditions over a finite time interval:

$$
\mathbf{c}(t+1)=\mathbf{A c}(t)+\mathbf{F}(t) \mathbf{q}(t), t=0,1,2, \ldots \tau
$$

How might this framework be used to represent the evolution of tracer concentrations in a system representative of the global ocean circulation? The simple four-box fluid transport model discussed in Section 2.4 provides a framework for discussion. In that model, the system state $\mathbf{c}(t)$ was defined by the homogeneous tracer concentrations in the two interior boxes. The evolution of the system state was prescribed by the state transition matrix, which described mass fluxes within and out of the system, and by the matrix $\mathbf{F}(t)$, which described mass fluxes into the system from two adjoining boundary boxes. The concentrations within the two boundary boxes were the controls $\mathbf{q}(t)$ on the problem; ignoring the transient initial state, the forward problem was completely specified by knowledge of $\mathbf{q}(t)$.

In the global circulation problem, the global ocean is represented by the discrete domain and fluxes derived in Gebbie and Huybers [2012], hereafter referred to as GH12. Section 2.5 discusses this work in greater detail. The global ocean below the mixed layer is represented by a 57,514-box model, and the ocean state vector $\mathbf{c}(t)$ consists of the tracer concentrations in each box. The controls are the concentrations in the 2806 surface grid boxes, each of which is identical to the concentrations in the underlying mixed layer grid boxes. The matrix $\mathbf{A}$ is a function of box volumes and inter-box volume fluxes and describes how tracer is transported in the model state. 
The matrix $\mathbf{F}$ describes how tracer is exported from the ocean mixed layer to the interior. Fluxes between and among elements of the state and control vectors are representative of the combined effects of advection and diffusion. Fluxes are constant in time, reflecting the statistical steady state solution specified by GH12.

The tracer inverse problem is described by Equation (2.22),

$$
\mathbf{y}(t)=\mathbf{B}(t) \mathbf{c}(t)+\mathbf{n}(t), t=0,1,2, \ldots \tau \text {. }
$$

In the paleoceanographic context, sediment core records are treated as noisy observations $\mathbf{y}(t)$ of a subset of the state vector. The matrix $\mathbf{B}(t)$ describes the subset of the state vector believed to be observed by sediment cores at a time $t$; the time dependence of $\mathbf{B}(t)$ reflects the fact that measurements in some records are not available at all times. The noise $\mathbf{n}(t)$ is defined as any part of the observational signal that does not originate from the transport of tracer by the modern steady-state circulation estimate. The noise can originate both from observational error (analytical error, diagenesis, chronological error, or other errors described in Chapter 3) and from deviations of the model mass circulation from the modern estimate. Inevitably, both sources of error will contribute.

The IBC problem may also be written in the whole-domain formulation of Equation (2.34) by creating vectors and matrices (denoted with bars) whose elements vary in both space and time:

$$
\overline{\mathbf{y}}=\overline{\mathbf{B}} \overline{\mathbf{G}}_{0} \mathbf{c}(0)+\overline{\mathbf{B}} \overline{\mathbf{G}} \overline{\mathbf{F}} \overline{\mathbf{q}}+\overline{\mathbf{n}} .
$$

To summarize the discussion in Section 2.3.3, $\overline{\mathbf{y}}$ is the whole-domain data vector, $\overline{\mathbf{B}} \overline{\mathbf{G}}_{0}$ describes the contribution (decaying in time) of the initial state vector $\mathbf{c}(0)$ to 
the data, $\overline{\mathbf{B}} \overline{\mathbf{G}} \overline{\mathbf{F}}$ relates the boundary conditions $\overline{\mathbf{q}}$ to the observations at all locations and times; and $\overline{\mathbf{n}}$ is the noise. The remainder of this section describes the model and addresses the construction of the matrices $\overline{\mathbf{B}}, \overline{\mathbf{F}}, \overline{\mathbf{G}}$, and $\overline{\mathbf{G}}_{0}$. In short, products of the matrices $\overline{\mathbf{G}}, \overline{\mathbf{G}}_{0}$, and $\overline{\mathbf{F}}$ are constructed from the state transition matrix $\mathbf{A}$ derived in $\mathrm{GH} 12$, and $\overline{\mathbf{B}}$ is determined by the times and locations of available sediment core records.

\subsubsection{Model specification}

Section 2.5 discusses the basis of the GH12 modern circulation estimate in detail. The GH12 model domain has 33 vertical levels and a $4^{\circ} \times 4^{\circ}$ resolution, giving a total of 74,064 grid boxes, 2806 of which lie at the ocean surface and 16550 of which lie above a prescribed mixed layer (defined locally based on observational estimates of winter mixed layer depth). The GH12 model is expected to deviate from the modern mass circulation in many ways, not least because steady fluxes are used to approximate the highly nonlinear and turbulent ocean, which is known to vary on all temporal and spatial scales. In particular, there is no annual cycle, which figures prominently in the subduction of tracers [Stommel, 1979, Marshall et al., 1993] and in high-latitude convection [Knauss, 1996]. Many properties of the mass circulation are not constrained by the available data and the GH12 estimate is intended here to represent a statistical steady state that is determined largely by time-variable processes. GH12 is a good choice for the problem at hand because it represents a plausible estimate of global tracer flux rates and because it was tuned specifically to represent the modern tracer distribution. However, any candidate circulation estimate (e.g. GCM output or other state estimates) could be used in the IBC problem, and future work should investigate the sensitivity of the results to this choice. 
The state vector $\mathbf{c}(t)$ is a time-varying $N$-vector where $N=74064-16550=57514$ (the number of grid boxes outside the ML). Each element of $\mathbf{c}(t)$ corresponds to the tracer concentration of one of the $N$ ocean grid boxes below the local mixed layer at the time $t$. Tracer concentrations are conservative in the model state, with the exception of small contributions from parameterized bottom flow; see GB10.

The control vector $\mathbf{q}(t)$ is a time-varying $L$-vector, where $L=2806$ and is equal to the number of surface grid boxes. Tracer concentrations within the mixed layer are assumed to be vertically uniform, and thus the mixed layer concentration is completely specified by the $L$ values describing concentrations at the surface.

The treatment of upwelling in the circulation model is important for long-term simulations of global ocean tracer transport. In the GH12 forward model, the mass circulation is closed, and fluid upwelling into the mixed layer compensates the flux into the interior at each time step. However, tracer concentration in the mixed layer boxes is not conserved; it is fixed by the controls at each time step. Thus if a delta function in space and time of tracer concentration were imposed in the mixed layer of the forward model, the tracer would propagate into the system state and the total tracer concentration there would converge to zero over long times as the tracer was gradually upwelled into the mixed layer and obliterated. This asymptotic, nonconservative behavior of the system is a consequence of nonconservative boundary conditions.

Alternatively, consider the tracer flux boundary conditions that are equivalent to the concentration boundary conditions (such a set always exists). To maintain a zero boundary concentration in the presence of upwelling positive tracer, as in the delta function case of the previous paragraph, there must be a compensating negative tracer flux at the boundary from the mixed layer grid boxes (that is, exterior to the ocean state). In the deglacial IBC problem, it is important to remember that $\delta^{18} \mathrm{O}_{c c}$ 
concentration boundary conditions reflect upwelling (both local and from the global mean glacioeustatic component) and mixed-layer transport (e.g. the spreading of buoyant plumes) as well as forcing by surface climate (e.g. precipitation, river runoff, or air-sea exchange).

The matrices $\overline{\mathbf{B}} \overline{\mathbf{G}}_{0}$ and $\overline{\mathbf{B}} \overline{\mathbf{G}} \overline{\mathbf{F}}$ in Equation (4.1) are constructed from Green functions following the adjoint procedure (Equation 2.36). Using the time tendency of $\mathbf{A}^{\top}$ augmented to include $\mathbf{F}$, eight forward runs of the adjoint model (corresponding to the 8 different core sites) were integrated for 5,000 years using MATLAB's ode15s stiff solver routine with variable time steps following a procedure similar to that of Gebbie [2012]. Initial conditions in all integrations were zero tracer concentration everywhere in the domain. In each of the eight integrations, a different Heaviside (in time) tracer concentration boundary condition was imposed at the locus of a sediment core record, and time series of tracer concentration in surface grid points was stored. The adjoint boundary Green function in the mixed layer is computed from the Heaviside response by differentiating in time using a centered difference scheme and interpolating to a spacing of 200 years. Finally, forward boundary Green functions are normalized from the adjoint Green functions by requiring that the time-asymptotic concentration at sediment core loci is 1 when subject to a uniform concentration boundary condition of 1 .

Heaviside responses are shown in Figures 4.2 and 4.3 to illustrate the propagation of tracer in the TMI steady state forward model. In each of these figures, large regions (illustrated in 4.1) that include deep water formation sites are "dyed" with a concentration of 1 at $t=0$ (i.e. a Dirichlet boundary condition) and held at that concentration for the rest of the integration. Each figure column shows the timeevolution of tracer concentration at a particular depth level.

In the North Atlantic forcing case, the tracer propagates within the Atlantic Ocean 


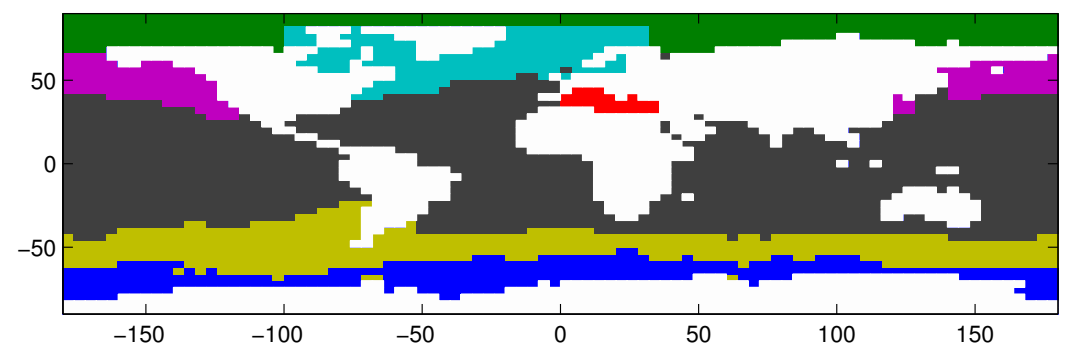

Figure 4.1: Seven surface regions defined in Gebbie and Huybers [2010]. Heaviside responses in Figures 4.2 and 4.3 were generated by enforcing uniform concentrations of 1 in the mixed layer of the North Atlantic (NATL, cyan), and the Antarctic (ANT, blue) regions.

from north to south, establishing a meridional gradient. There is visual evidence for preferential transport of tracer along western margins and along the Equator in the Atlantic. At 4,000 years, when the tracer distribution is near equilibrium, meridional gradients are sharpest at 2000 and $3000 \mathrm{~m}$ where the Atlantic Ocean joins the Southern Ocean, while at $1000 \mathrm{~m}$ the gradient is more moderate. The tracer plume in the Pacific Ocean moves northward from the Southern Ocean and eventually establishes a meridional gradient. Equilibrium tracer concentrations in the Pacific Ocean are of order 0.1 and increase with depth between 1000 and $3000 \mathrm{~m}$.

In the Southern Ocean forcing case, tracer signals penetrate to depth and propagate northward, with local maxima in the Ross and Weddell Seas. Tracer concentrations in the Atlantic are modest, and at equilibrium a sharp gradient appears at the latitude of Cape Horn that is complementary to that observed in the Atlantic forcing case. At equilibrium outside of the Atlantic sector, meridional tracer gradients are sharpest at $1000 \mathrm{~m}$, and tracer concentrations in the deep North Pacific are roughly 0.5 , again with a local maximum at depth.

There are many additional suggestive features in these plots and a complete description is beyond the scope of this work. Clearly evident, however, is the complexity and three-dimensional anisotropy of the evolving tracer plume, with evidence 


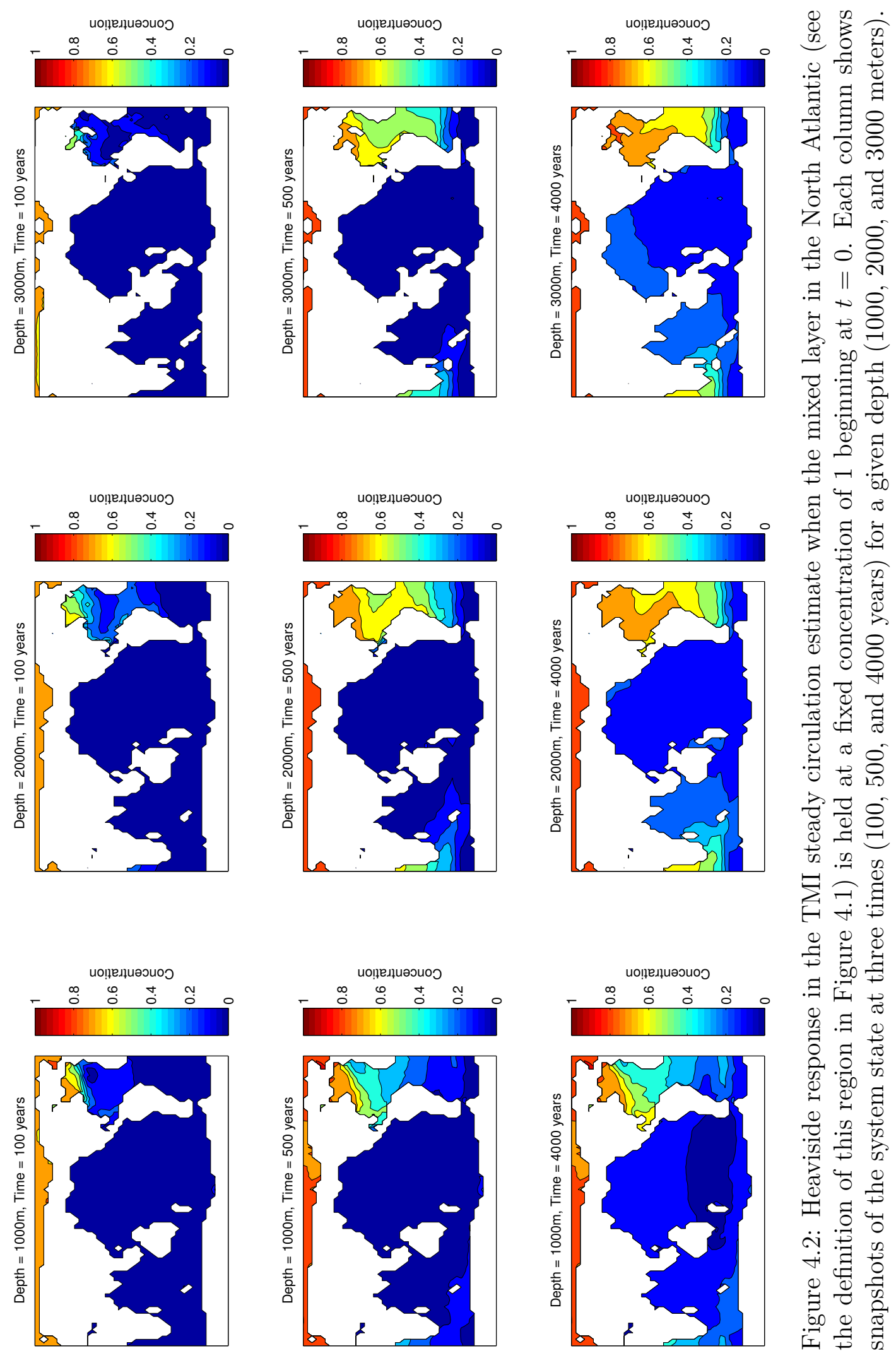



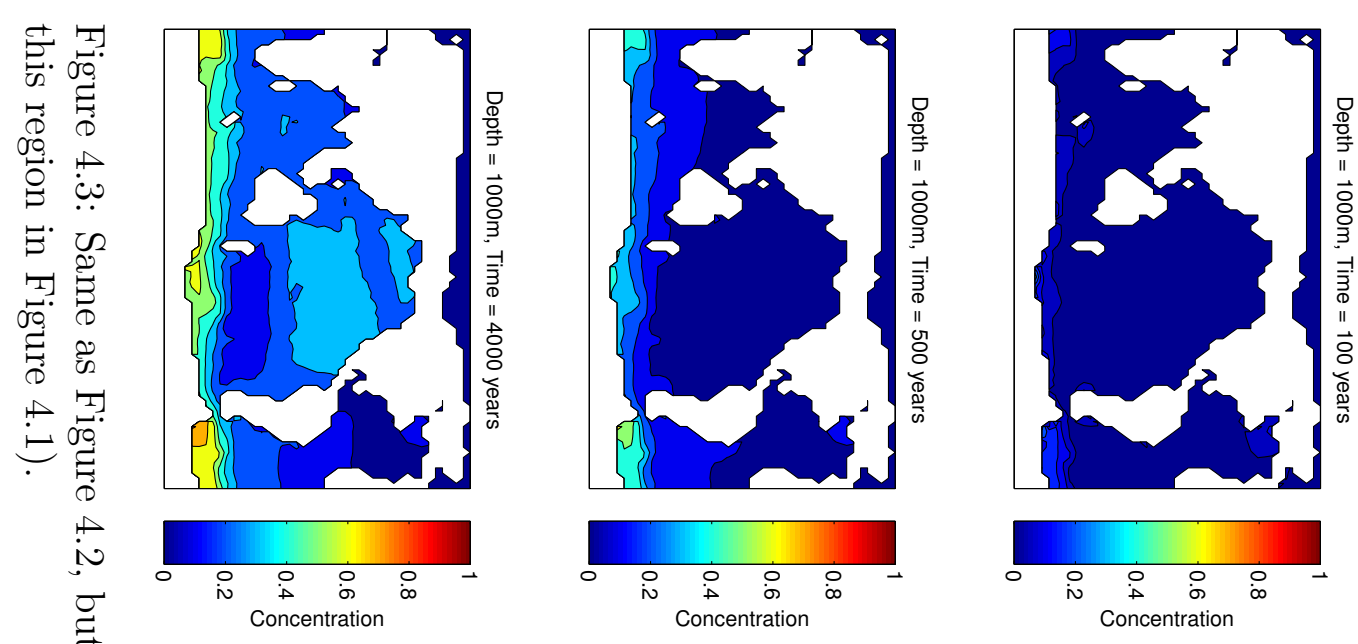

$\stackrel{\circ}{\circ}$

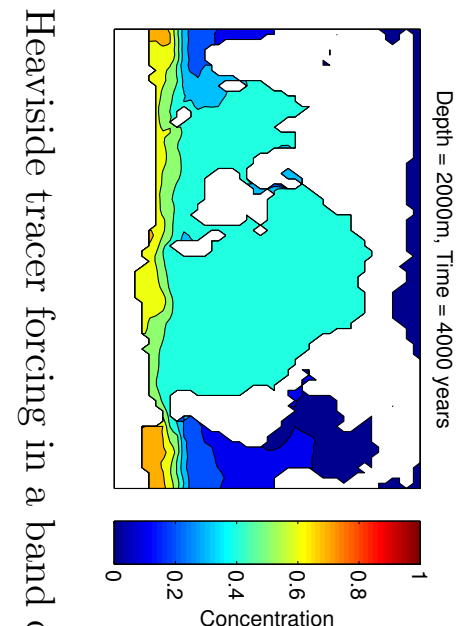

官
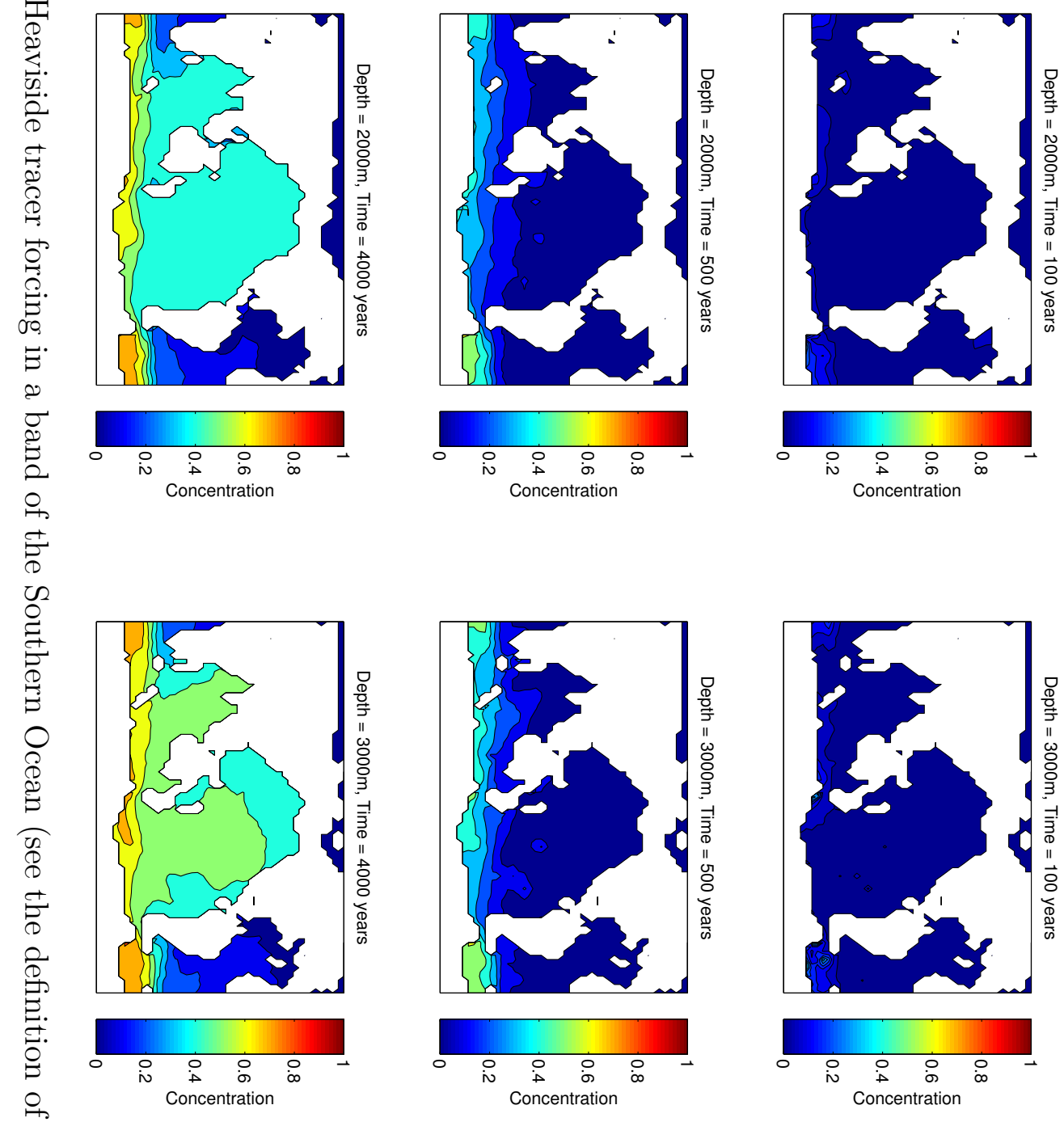
for transport from both advective and diffusive properties. The Heaviside responses, which show the pathways for transmission of surface climate signals to depth, illustrate some of the challenges of the tracer inverse problem. Comparisons with tracer concentration forcings in other surface regions (not shown) show that mixed layer concentration signals are transmitted to depth, where they might be observed by benthic observations, most rapidly and with least distortion when they originate in areas of high deep water formation rates.

In the deglacial inverse problem, the time step $\Delta t$ is set to 200 years and the number of time steps $\tau$ is 101, corresponding to the 20,000 year reconstruction interval 25,000 ybp $-5,000$ ybp, i.e. $t=-25000,-24800, \cdots-5200,-5000$ chosen to resolve the deglacial transition (roughly -20000 to -8000 years). The time step was chosen to reflect the resolution of benthic time series and so as to resolve millennial-scale variability. After 5,000 years, the boundary Green functions are set to zero, which is accurate to within $5 \times 10^{-5}$. This approximation may lead to errors in solutions of the steady IBC problem and should be revisited in future work.

It is now possible to specify the dimensions in the statement of the IBC problem,

$$
\overline{\mathbf{y}}=\overline{\mathbf{B}} \overline{\mathbf{G}}_{0} \mathbf{c}(0)+\overline{\mathbf{B}} \overline{\mathbf{G}} \overline{\mathbf{F}} \overline{\mathbf{q}}+\overline{\mathbf{n}} .
$$

The whole-domain $\overline{\mathbf{y}}$ is an 785 -vector, $\overline{\mathbf{B}}$ is an $785 \times(101 * 57,514)$ matrix describing elements of the state vector observed by the data, $\overline{\mathbf{G}}_{0}$ is a $(57,514 * 101) \times 57,514$ matrix that describes contributions to the system state at different times due to the initial conditions, $\mathbf{c}(0)$ is a 57,514 -vector of (unknown) initial conditions, $\overline{\mathbf{G}}$ is a $(57,514 * 101) \times(57,514 * 101)$ matrix describing the evolution of the system state, $\overline{\mathbf{F}}$ is a $(57,514 * 101) \times(2806 * 101)$ matrix describing the contributions to the system state from the boundary conditions, $\overline{\mathbf{q}}$ is a $(2806 * 101)$-vector of (unknown) 
boundary conditions, and $\overline{\mathbf{n}}$ is an 785 -vector of observational noise. Luckily from a computational perspective, it is possible to construct just the products $\overline{\mathbf{B}} \overline{\mathbf{G}_{0}} \overline{\mathbf{F}}$, which is $785 \times(2806)$, and $\overline{\mathbf{B}} \overline{\mathbf{G}} \overline{\mathbf{F}}$, which is $785 \times(101 * 2806)$, and to use the sparsity of these matrices to make the inverse problem much more tractable.

Omitting the contribution from initial conditions, the problem appears to be highly underdetermined: 785 noisy observations are being used to constrain 785 noise observations and $2806 * 101$ controls.

\subsubsection{Treatment of benthic $\delta^{18} \mathrm{O}_{c c}$ data in the inverse problem}

The fluctuating part of the $i^{t h}$ record is defined by subtracting an estimate $\tilde{m}_{i}$ of the record mean,

$$
\begin{aligned}
\mathbf{f}_{i} & =\mathbf{y}_{i}-\mathbf{d} \tilde{m}_{i} \\
\mathbf{d} & =[1,1, \ldots 1]^{\top},
\end{aligned}
$$

where the $\tilde{m}_{i}$ are least-squares estimates of record means and the $\mathbf{y}_{i}$ are the objectively mapped records of $\delta^{18} \mathrm{O}_{c c}$ (described in Chapter 3). The whole-domain vectors $\overline{\mathbf{y}}$ and $\overline{\mathbf{f}}$ were constructed by concatenating the $\mathbf{y}_{i}$ and $\mathbf{f}_{i}$ to yield vectors that vary in both space and time:

$$
\begin{aligned}
& \overline{\mathbf{y}}=\left[\mathbf{y}_{1}, \mathbf{y}_{2}, \ldots \mathbf{y}_{9}\right] \\
& \overline{\mathbf{f}}=\left[\mathbf{f}_{1}, \mathbf{f}_{2}, \ldots \mathbf{f}_{9}\right] .
\end{aligned}
$$

The matrix $\mathbf{B}(t)$ has 74,064 columns and a number of rows corresponding to the number of available sediment cores at a given time. Each row $\mathbf{B}_{i}=\delta_{j, T M I_{i}}$, where $T M I_{i}$ is the index of the location of the $i^{t h}$ sediment core as represented in the TMI 


\begin{tabular}{lcccccccc} 
Name & Depth & TMI Depth & Lat & TMI Lat & Lon & TMI Lon & Start (ybp) & End (ybp) \\
\hline SA1967 & 1967 & 2000 & -23.32 & -24 & 12.38 & 10 & 23,800 & 5,200 \\
NA3223 & 3223 & 3000 & 12.4 & 12 & -18.1 & -18 & 24,800 & 5,400 \\
NA1299 & 1299 & 1300 & 12.08 & 12 & -61.25 & -62 & 24,800 & 5,200 \\
SA3770 & 3770 & 4000 & -44.15 & -48 & -14.23 & -18 & 24,800 & 5,200 \\
EI2100 & 2100 & 2000 & -9.65 & -12 & 118.34 & 118 & 24,800 & 5,200 \\
NA3146 & 3146 & 3000 & 37.8 & 36 & -10.17 & -10 & 24,800 & 5,200 \\
NI1580 & 1580 & 1500 & 10.77 & 12 & 51.95 & 50 & 24,800 & 5,200 \\
EP3210 & 3210 & 3000 & -3.6 & -4 & -84 & -86 & 24,800 & 5,200 \\
\hline
\end{tabular}

Table 4.1: Properties of the data as used in the inverse model. Locations of sediment cores were interpolated to TMI ocean grid box locations by minimizing differences in depth, longitude, and latitude.

state vector (Table 4.1). In several cases there are offsets of 200-300 m between model grid boxes and core depths; future work should correct these offsets by interpolating between grid boxes.

\subsection{Results}

Results are described for two IBC problems. First, the initial conditions for the mean-removed problem are estimated by seeking a steady set of boundary conditions $\tilde{\mathbf{q}}_{0}$ that best matches the average of the interval $[-25000,-20000]$ in each record, nominally representative of the LGM. Next, a time-varying solution $\tilde{\overline{\mathbf{q}}}$ is sought that best matches the mean-removed records.

\subsubsection{Solution to the "LGM equilibrium" problem}

To solve the initial value problem, we seek a steady solution of controls $\tilde{\mathbf{q}}_{0}$ that best reconstructs the average values of the mean-removed sediment core records $\overline{\mathbf{f}}$ over the shorter interval $[-25000,-20000]$ in a least-square sense. The initial state vector estimate $\tilde{\mathbf{c}}(0)$ is defined as the system state that is in equilibrium with the 
steady boundary conditions $\tilde{\mathbf{q}}_{0}$. Notably, since the initial conditions have no effect on the system state after -20000, they have no impact on the deglacial interval [-20000, -8000] that is of primary interest here.

The statement of the initial value problem is

$$
\mathbf{y}_{0}=\mathbf{B}_{0} \mathbf{G}_{0} \mathbf{F} \mathbf{q}_{0}+\mathbf{n},
$$

where $\mathbf{y}_{0}$ is an 8-vector consisting of the mean values of the fluctuating parts of the sediment cores on $[-25000,-20000] ; \mathbf{B}_{0} \mathbf{G}_{0} \mathbf{F}$ is a $8 \times 2806$ matrix that describes the steady tracer problem at the model locations corresponding to the core sites, $\mathbf{q}_{0}$ is an unknown 2806-vector of mixed layer tracer concentration, and $\mathbf{n}_{0}$ is an unknown 8-vector of observational noise. No row weighting was applied. The solution is given by

$$
\tilde{\mathbf{q}}_{0}=\sum_{i=1}^{K_{0}^{\prime}} \frac{\mathbf{u}_{0 i}^{\top} \mathbf{y}_{0}}{\lambda_{0 i}} \mathbf{v}_{0 i}+\sum_{i=K_{0}^{\prime}+1}^{2806} \alpha_{0 i} \mathbf{v}_{0 i} .
$$

Here, the nullspace parameter is set to $K_{0}^{\prime}=3$ and the nullspace contributions are set to zero. The nullspace parameter was chosen to be the lowest number that fit the data roughly to within $0.2 \%$ (specifically, the $K^{\prime}=2$ case had substantially larger residual amplitudes $\left.\tilde{\mathbf{n}}_{0}\right)$.

Figure 4.4 plots the solution $\tilde{\mathbf{q}}_{0}$ to the initial value problem as a map of surface $\delta^{18} \mathrm{O}_{c c}$. The solution is everywhere positive but is extremely sparsely distributed. Fundamentally this occurs because the solution is a set of sums and differences of the adjoint Green functions calculated at each core site, each of which is heavily weighted towards regions of deepwater formation in the model of GH12 (see 4.16 and the discussion in Section 4.5). A single maximum in the Weddell Sea accounts for most of the variance in $\tilde{\mathbf{q}}_{0}$, but there are nonzero values distributed throughout the 


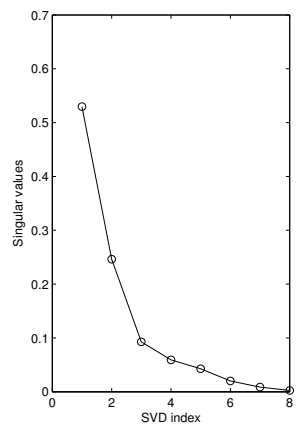

(a)

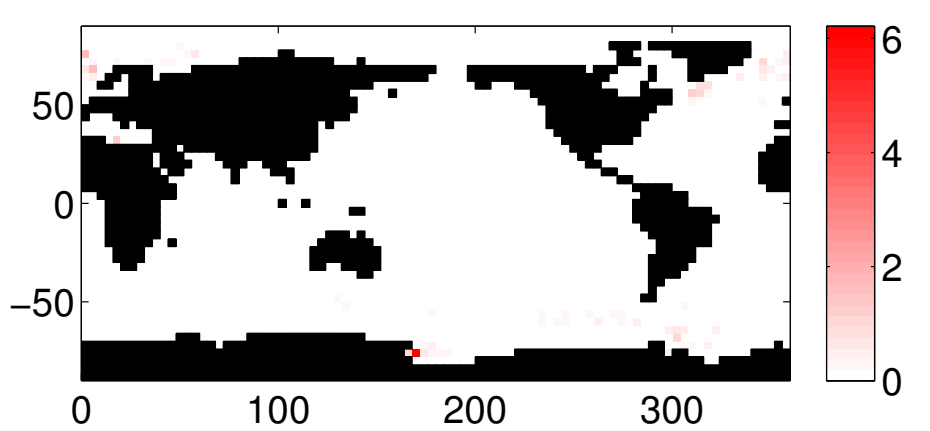

(b)

Figure 4.4: The solution to the initial value problem. Panel 4.4a shows the singular values of the initial value problem and panel $4.4 \mathrm{~b}$ plots the solution $\tilde{\mathbf{q}}_{0}$. A single maximum in the Weddell Sea accounts for most of the variance, but there are nonzero values distributed throughout the North Atlantic, Arctic, and Southern Oceans. Units of both plots are $\%_{0} \delta^{18} \mathrm{O}$.

\begin{tabular}{lccc} 
Name & $\mathbf{y}_{0}(\%)$ & $\tilde{\mathbf{q}}_{0}(\%)$ & $\tilde{\mathbf{n}}_{0}(\%)$ \\
\hline SA1967 & 0.96 & 1.02 & 0.06 \\
NA3223 & 0.78 & 0.70 & -0.08 \\
NA1299 & 0.68 & 0.70 & 0.03 \\
SA3770 & 0.92 & 0.70 & -0.21 \\
EI2100 & 0.72 & 0.70 & -0.02 \\
NA3146 & 0.84 & 0.79 & -0.06 \\
NI1580 & 0.50 & 0.71 & 0.21 \\
EP3210 & 0.55 & 0.62 & 0.07 \\
\hline
\end{tabular}

Table 4.2: Data, reconstructed data, and residuals for the initial value problem.

North Atlantic, Arctic, and Southern Oceans. Values of $\mathbf{y}_{0}, \tilde{\mathbf{q}}_{0}$, and $\tilde{\mathbf{n}}_{0}$ are listed in Table 4.2 .

\subsubsection{Solution to the fluctuating problem}

\section{Problem setup}

To solve the fluctuating problem, we seek the time-varying solution of controls $\tilde{\overline{\mathbf{q}}}$ that best reconstructs the time-varying components $\overline{\mathbf{f}}$ of the sediment core records in 
a least-squares sense on the interval $[-25000,-5000]$. In all cases, the effect of the initial conditions was removed from the data by subtracting $\overline{\mathbf{B}} \overline{\mathbf{G}}_{0} \tilde{\mathbf{q}}_{0}$ from $\overline{\mathbf{f}}$ before scaling; this component was added back to the reconstructed data before plotting. A whole-domain row weighting matrix $\overline{\mathbf{W}}$ was constructed as a block-diagonal matrix of the uncertainty matrices $\mathbf{P}_{i}$ of the eight sediment core records as defined in Equation 3.6 ,

$$
\overline{\mathbf{W}}=\left[\begin{array}{cccc}
\mathbf{P}_{1} & \mathbf{0} & \ldots & \mathbf{0} \\
\mathbf{0} & \mathbf{P}_{2} & \ldots & \mathbf{0} \\
\vdots & \vdots & \ddots & \vdots \\
\mathbf{0} & \mathbf{0} & \ldots & \mathbf{P}_{9}
\end{array}\right]
$$

The prior noise statistics are spectrally red in time because of non-zero off-diagonal terms in the $\mathbf{P}_{i}$ (Section 3.3.3). Measurement errors between records are believed, a priori, to be uncorrelated at all times as specified by the zero off-diagonal block matrix elements of $\overline{\mathbf{W}}$.

The statement of the fluctuating IBC problem is, after applying the row weighting,

$$
\overline{\mathbf{W}}^{-T / 2} \overline{\mathbf{f}}=\left[\overline{\mathbf{W}}^{-T / 2} \overline{\mathbf{B}} \overline{\mathbf{G}} \overline{\mathbf{F}}\right] \overline{\mathbf{q}}+\overline{\mathbf{W}}^{-T / 2} \overline{\mathbf{n}}
$$

where $\overline{\mathbf{W}}^{-\top / 2} \overline{\mathbf{f}}$ is the scaled 785 -vector of fluctuating sediment core records, $\left[\overline{\mathbf{W}}^{-\top / 2} \overline{\mathbf{B}} \overline{\mathbf{G}} \overline{\mathbf{F}}\right]$ is a $785 \times(2806 * 101)$ matrix relating controls at all locations $(L=2806)$ and times $(\tau=101)$ to the state vector at the core sites, and $\overline{\mathbf{W}}^{-T / 2} \overline{\mathbf{n}}$ is an 785 -vector of scaled observational noise. Define the singular value decomposition to be

$$
\mathbf{U} \boldsymbol{\Lambda} \mathbf{V}^{\top}=\left[\overline{\mathbf{W}}^{-\top / 2} \overline{\mathbf{B}} \overline{\mathbf{G}} \overline{\mathbf{F}}\right]
$$

As before, the SVD solution is 


$$
\tilde{\overline{\mathbf{q}}}=\sum_{i=1}^{K^{\prime}} \frac{\mathbf{u}_{i}^{\top} \overline{\mathbf{f}}}{\lambda_{i}} \mathbf{v}_{i}+\sum_{i=K^{\prime}+1}^{2806 * 101} \alpha_{i} \mathbf{v}_{i}
$$

and setting the $\alpha_{i}=0$ yields the particular SVD solution. Figure 4.5 shows the singular values and weights on the fluctuating SVD problem. The decay of the singular values of $\left[\overline{\mathbf{W}}^{-\top / 2} \overline{\mathbf{B}} \overline{\mathbf{G}} \overline{\mathbf{F}}\right]$ is gradual and hundreds of singular values are of order 1 , suggesting that many structures in the range vectors $\mathbf{v}_{i}, i \leq K^{\prime}$ are resolvable by the data (Figure 4.5). Plateaus in the plot of ranked singular values correspond to structures in space that are resolvable at a range of frequencies. Panel 4.5b, which

plots $\frac{\mathbf{u}_{i}^{\top} \overline{\mathbf{f}}}{\lambda_{i}}$ and $\mathbf{u}_{i}^{\top} \overline{\mathbf{f}}$, show that the pattern of data projection onto singular vectors is complex, with strong projections appearing in groups near SVD indices 0, 190, 290, and 420. Solutions were obtained for a variety of choices of $K^{\prime}$; here the $K^{\prime}=300$ and $K^{\prime}=400$, cases are discussed.

\section{A note about plotting whole-domain vectors}

Solutions to the fluctuating problem are three-dimensional fields (two spatial dimensions and a time dimension) and can be thought of as 20,000-year long movies of surface mixed layer concentration variability. To give some sense of the evolution of the fields (rather than, for instance, just plotting snapshots at various times), the solutions $\tilde{\overline{\mathbf{q}}}$ are singular value decomposed into what are known in meteorology as EOF-PC pairs [e.g. Bretherton et al., 1992]. It should be stressed that this additional SVD procedure is distinct from the procedure used to obtain the solution and is just a convenient way of plotting the results.

The EOF decomposition is effected by reordering the whole-domain vector $\tilde{\overline{\mathbf{q}}}$ into a matrix $\tilde{\mathbf{Q}}$ whose rows index spatial locations and whose columns index time. Thus Q is a $L \times \tau$ matrix, where $L=2806$ and $\tau=101$. The singular value decomposition 


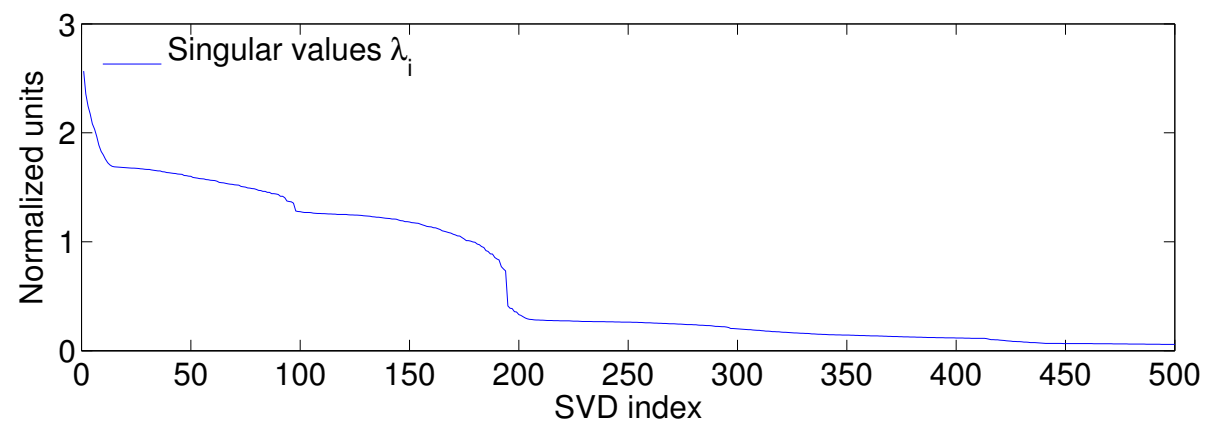

(a)

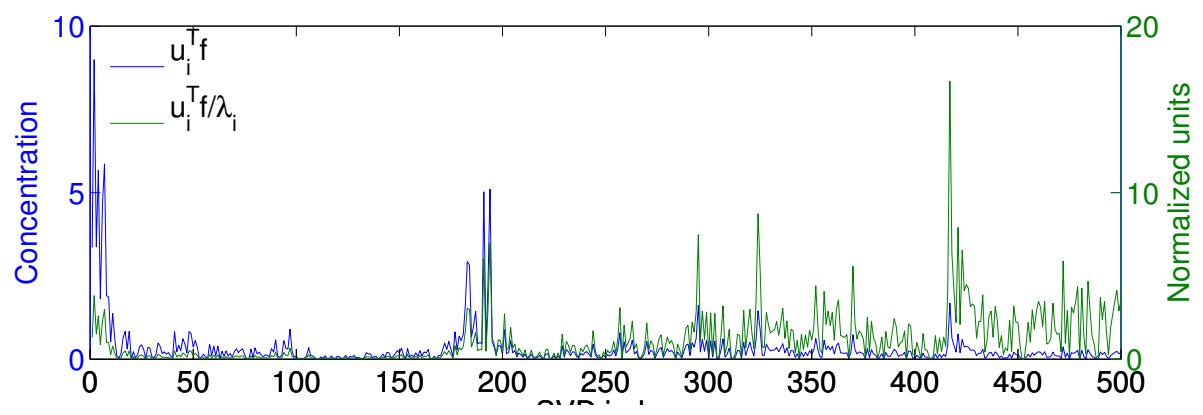

(b)

Figure 4.5: SVD solution range vectors are weighted by $\frac{\mathbf{u}_{i}^{\top} \overline{\mathbf{f}}}{\lambda_{i}}$ (Equation (4.2)) and thus reflect both the singular values $\lambda_{i}$ (panel 4.5a) and the projection $\mathbf{u}_{i}^{\top} \overline{\mathbf{f}}$ of the meanremoved records onto the left singular values of the weighted matrix $\left[\overline{\mathbf{W}}^{-\mathrm{T} / 2} \overline{\mathbf{B}} \overline{\mathbf{G}} \overline{\mathbf{F}}\right]$. The number of singular values is 785 , corresponding to the minimum dimension of $\left[\overline{\mathbf{W}}^{-\mathrm{T} / 2} \overline{\mathrm{B}} \overline{\mathrm{G}} \overline{\mathbf{F}}\right]$. 
of $\tilde{\mathbf{Q}}$ is

$$
\tilde{\mathbf{Q}}=\mathbf{U}_{q} \boldsymbol{\Lambda}_{q} \mathbf{V}_{q}^{\top}
$$

where the $\mathbf{u}_{q i}$ are orthonormal 2806-vectors (the EOFs) describing spatial patterns, the $\mathbf{v}_{q i}$ are orthonormal 101-vectors (the PCs) that weight the corresponding EOFs at each time step, and the singular values on the diagonal of $\boldsymbol{\Lambda}_{q}$ are the amplitudes (in units of concentration) weighting each EOF-PC pair. In the case when a subset of singular values contain most of the variance in the field, a truncated representation of $\tilde{\mathbf{Q}}$ consisting of a few EOF-PC pairs can be a very good approximation.

In the results discussed in this section, very good representations of the solutions can be obtained with only several EOF-PC pairs. This is not immediately intuitive given that solutions $\tilde{\overline{\mathbf{q}}}=\sum_{i=1}^{K^{\prime}} \frac{\mathbf{u}_{i}^{\top} \overline{\mathbf{f}}}{\lambda_{i}} \mathbf{v}_{i}$ are the sum of many orthogonal components (the range vectors $\mathbf{V}$ of the IBC problem). However, consider two whole-domain vectors that are orthogonal in space but not time (for instance, two orthogonal spatial patterns varying in time by the same annual cycle). These whole-domain vectors are orthogonal, but their sum can be expressed as a single EOF-PC pair (the sum of the two spatial patterns, varying in time as the annual cycle). The SVD solution is such a sum of whole-domain vectors that are orthogonal in space and time but that have many spatial structures in common (specifically, the spatial patterns dictated by the surface expression of adjoint Green functions computed at sediment core sites).

\section{Comparison of solutions with $K^{\prime}=300$ and $K^{\prime}=400$}

Figures 4.6 and 4.7 show solutions $\tilde{\overline{\mathbf{q}}}+\tilde{\mathbf{q}}_{0}$ to the fluctuating IBC problem $\left(\tilde{\mathbf{q}}_{0}\right.$ is the contribution from the initial condition problem) for choices of nullspace parameter $K^{\prime}=300$ and $K^{\prime}=400$, respectively. Henceforth these solutions will be referred to as $\tilde{\mathbf{q}}_{300}$ and $\tilde{\mathbf{q}}_{400}$. 
At every space and time, the amplitude of the concentration is the product of the corresponding location in the EOF and time in the PCs. As always, it is important not to interpret EOF-PC pairs (EPPs) as dynamically independent modes of the physical system; here, the decomposition is merely a convenient way of plotting the solutions. In both solutions discussed, the first three EPPs account for over $90 \%$ of the solution variance.

All solutions have nonzero values in regions of the GH12 model that export volume below the mixed layer. The discussions of the solution resolution in Sections 4.4.1 and 4.5 help to understand this feature of the solutions.

The leading PC in $\tilde{\overline{\mathbf{q}}}_{300}$, representing $50 \%$ of the solution variance, is a smooth curve (the high frequencies are smaller than the standard error of the solutions and can be disregarded) with a minimum value of roughly $-6 \%$ at -19000 years, climbing over an 11 kyr period to a maximum value of roughly $8 \%$ shortly before -8000 years. The decay to zero at -5000 is an edge effect ${ }^{1}$. The corresponding spatial pattern is everywhere negative, with a pattern of values over the North Atlantic and Arctic similar to those seen in the solutions of the mean and initial value problems, though with reduced maxima in the Weddell Sea. The second EPP pair deviates strongly from zero in intervals centered on $[-17000,-16000]$ and -8200 with a spatial pattern dominated by a gradient of values in the model North Atlantic and Arctic. The third EPP consists of relatively low-amplitude, low frequency variations.

Solutions obtained for $K^{\prime}=300$ and $K^{\prime}=400$ differ by the addition of 100 additional right singular vectors $\mathbf{v}_{301}, \mathbf{v}_{302}, \ldots \mathbf{v}_{400}$ weighted by the corresponding $\frac{\mathbf{u}_{i}^{\top} \overline{\mathbf{f}}}{\lambda_{i}}$. As such $\tilde{\overline{\mathbf{q}}}_{400}$ has greater variance (by over a factor of 2) and greater uncertainty (discussed in the next section) than $\tilde{\overline{\mathbf{q}}}_{300}$. Analysis of the difference of the two solutions

\footnotetext{
${ }^{1}$ At -5000 and times shortly before, there is very little information about the controls because insufficient time has passed for tracer concentration values therein to propagate to the loci of observations.
} 
(not shown) shows that the additional variability is approximately represented by first EPP pair of $\tilde{\mathbf{q}}_{400}$. The leading EOF of $\tilde{\overline{\mathbf{q}}}_{400}$ is dominated by a zonal gradient in the Southern Ocean and predominantly positive contributions in the Northern Hemisphere. The corresponding PC shows a large, negative excursion in the middle third of the interval with peaks centered on $-15.5 \mathrm{kyr}$ and $-12.5 \mathrm{kyr}$. The second EPP of $\tilde{\mathbf{q}}_{400}$ has structure and amplitude similar to the first EPP pair of $\tilde{\mathbf{q}}_{300}$. The third PC has a broad excursion centered on $[-17000,-16000]$ and a sharp peak at -8200 .

Because the spatial structure of these solutions is dominated by deepwater formation regions, the physical meaning of the solutions is not clear. A particular cause for concern are the negative $\delta^{18} \mathrm{O}_{c c}$ values inferred at some surface locations. Implications of this result are addressed in Section 4.5.

Nevertheless, some features in the principal components are suggestive of deglacial features and are worthy of mention. The PC1 of $\tilde{\mathbf{q}}_{300}$ and PC2 of $\tilde{\mathbf{q}}_{400}$ describe a canonical, gradual picture of the deglaciation in terms of benthic $\delta^{18} \mathrm{O}_{c c}$. The twopeaked excursion in PC1 $\tilde{\mathbf{q}}_{400}$ may be suggestive of the Bolling Allerod / Younger Dryas transition at some locations. Large peaks are visible at $-8.2 \mathrm{kyr}$ in PC2 of $\tilde{\overline{\mathbf{q}}}_{300}$ and PC3 of $\tilde{\mathbf{q}}_{400}$; these may be due to the 8.2 kyr event (hypothesized to have been from catastrophic draining of Laurentide lakes; e.g. Barber et al. [1999]).

The raw (mean-removed) observations, objectively mapped data $\overline{\mathbf{f}}$, observational uncertainty, and reconstructed data $\tilde{\overline{\mathbf{f}}}$ are plotted as time series in Figures 4.8 and 4.9 for $K^{\prime}=300$ and $K^{\prime}=400$, respectively. The $K^{\prime}=400$ case necessarily produces a superior reconstruction, at the expense of higher solution amplitudes and uncertainties. In particular, $\tilde{\mathbf{q}}_{400}$ does substantially better at matching EP3210 during the deglacial transition as well as NA1299 around -15000, though SA1967, which is reconstructed poorly, is not much improved and the estimate for NI1580 has additional high-frequency variability in the residual for $\tilde{\mathbf{q}}_{400}$. The origin for the $8.2 \mathrm{kyr}$ peak 

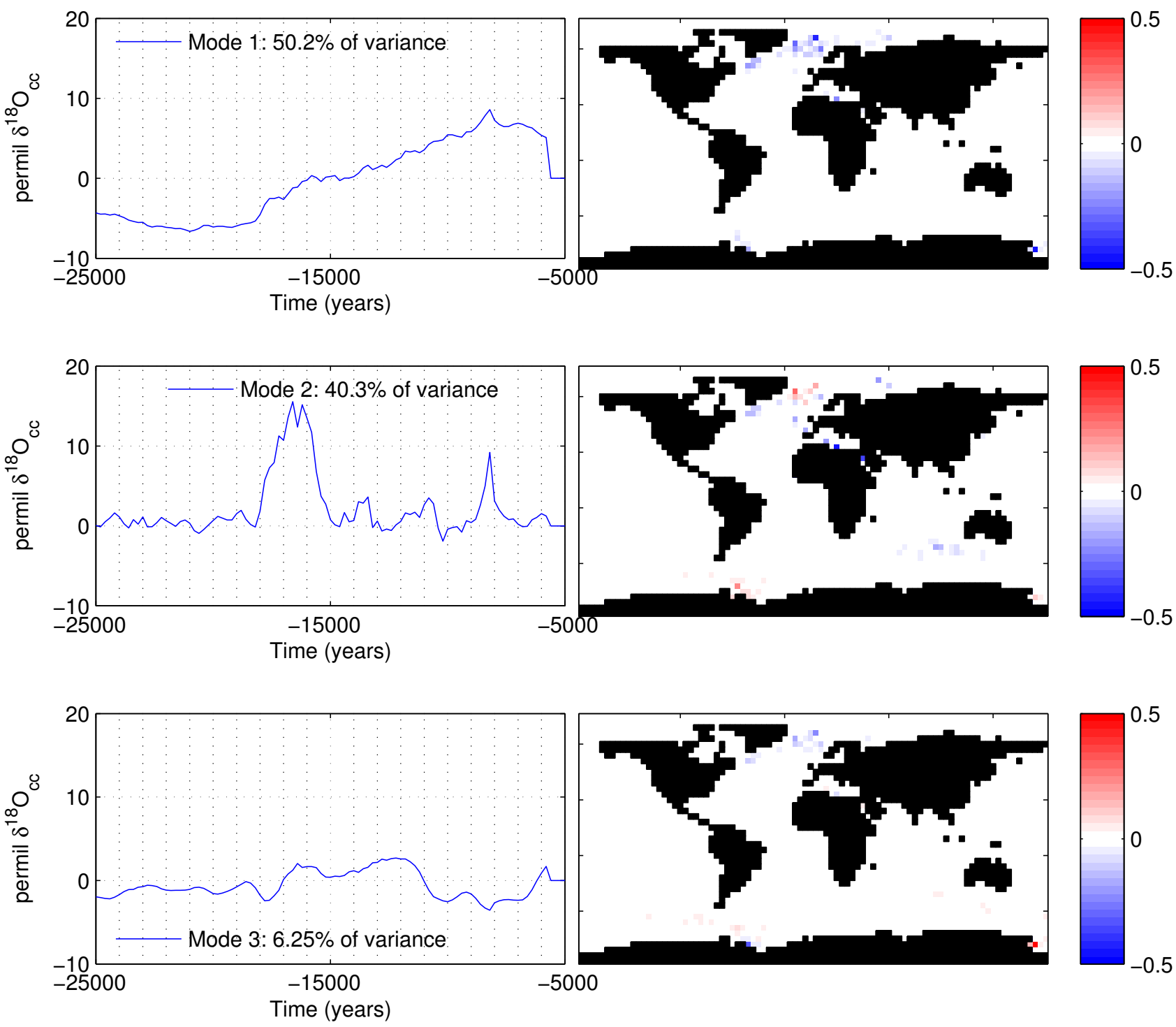

Figure 4.6: The solution $\tilde{\mathbf{q}}$ obtained with effective nullspace parameter $K^{\prime}=300$ for the time evolution of "fluctuation" (mean-removed) surface $\delta^{18} \mathrm{O}_{c c}$ is plotted as three EOF-PC pairs. EOF-PC pairs are computed by finding the singular value decomposition of the whole-domain solution vector, reordered into a matrix indexed by space in rows and time in columns (see Section ). The EOFs (spatial patterns, right panels) are shown in normalized units, while the PCs (time series, left panels) have the units of the tracer. Times and regions of zero value lie in the solution null space of the problem. The solution has low values everywhere at the end of the reconstruction interval because of lags in transporting tracer to observable regions. 

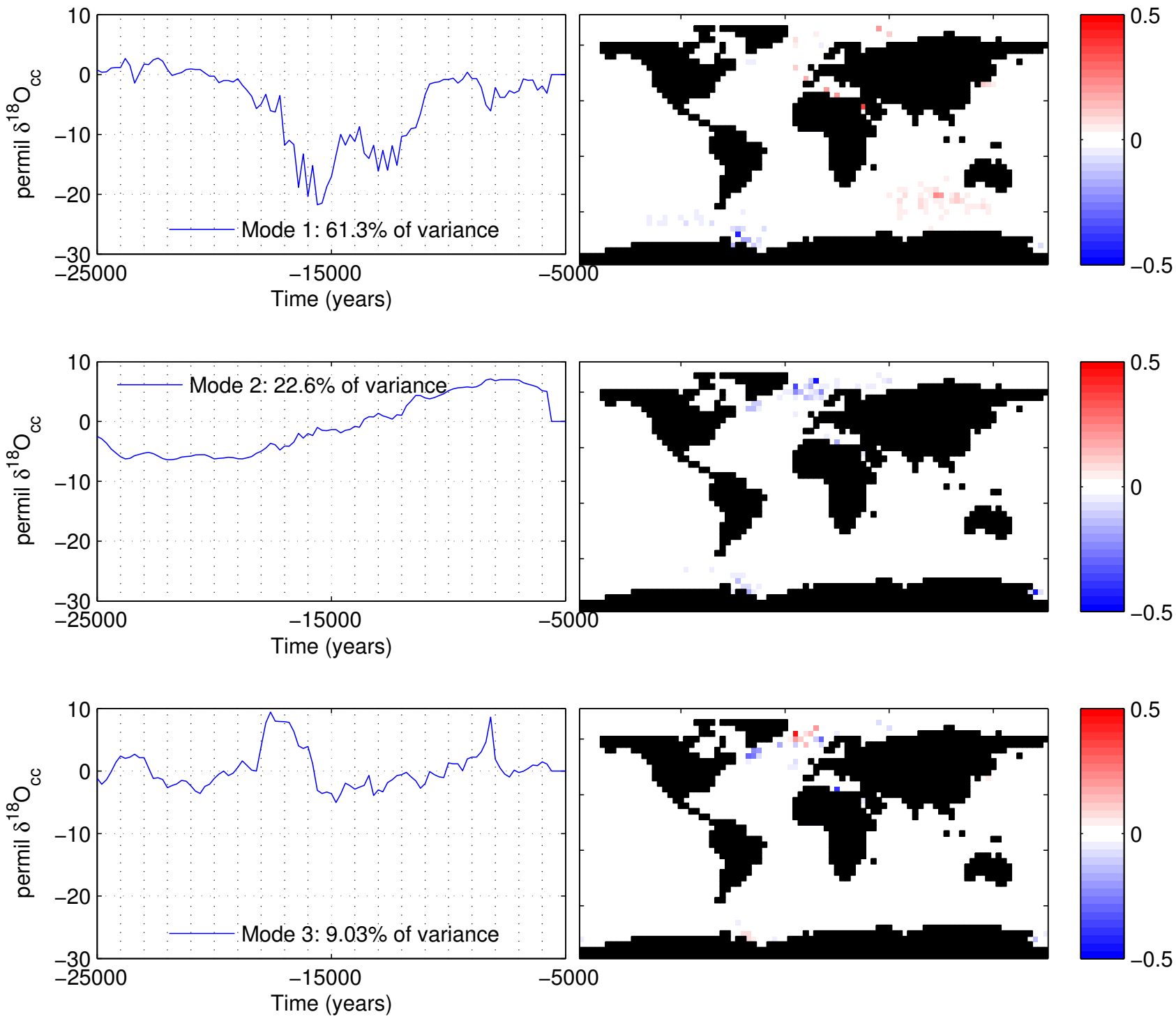

Figure 4.7: Same as Figure 4.6, but for $K^{\prime}=400$. 
in both solutions can be seen in NA1299 and NA1580, each of which has a negative excursion near that time.

\subsection{Further discussion of the $K^{\prime}=400$ case}

For the sake of brevity, further properties of the solution will be discussed only for the $K^{\prime}=400$ case, which was chosen because it reproduces the data qualitatively well (in particular, the $K^{\prime}=400$ reconstructs the phase lag between NA3146 and EP3210, which motivated the studies of Skinner and Shackleton [2005], Wunsch and Heimbach [2008], and Gebbie [2012]). This section evaluates the uncertainty of the solution and the statistics of the residuals.

\subsubsection{Solution uncertainty and resolution}

The uncertainty of the particular SVD solution is given by Equation (2.14):

$$
\mathbf{P}=\mathbf{C}_{\tilde{\mathbf{q}} \tilde{\mathbf{q}}}+\mathbf{Q}_{v}\left\langle\boldsymbol{\alpha} \boldsymbol{\alpha}^{\top}\right\rangle \mathbf{Q}_{v}^{\top}
$$

where the solution covariance matrix $\mathbf{C}_{\tilde{\mathbf{q}} \tilde{\mathbf{q}}}$ is a square, sparse matrix with dimensions $(101 * 2806) \times(101 * 2806), \mathbf{Q}_{v}$ is the $(2806 * 101) \times\left(2806 * 101-K^{\prime}\right)$ matrix of

solution nullspace vectors, and $\left\langle\boldsymbol{\alpha} \boldsymbol{\alpha}^{\top}\right\rangle$ is the $\left(2806 * 101-K^{\prime}\right) \times\left(2806 * 101-K^{\prime}\right)$ covariance matrix of nullspace weights. The total uncertainty $\mathbf{P}$ may thus be thought of as having two parts, one $\left(\mathbf{C}_{\tilde{\mathbf{q}} \tilde{\mathbf{q}}}\right)$ arising from observational uncertainties and the other $\left(\mathbf{Q}_{v}\left\langle\boldsymbol{\alpha} \boldsymbol{\alpha}^{\top}\right\rangle \mathbf{Q}_{v}^{\top}\right)$ arising from contributions of the solution nullspace vectors, which are omitted in the particular solution. The first of these components may be estimated; the second is harder to quantify, but the solution resolution matrix $\mathbf{T}_{v}$ provides some insight. 

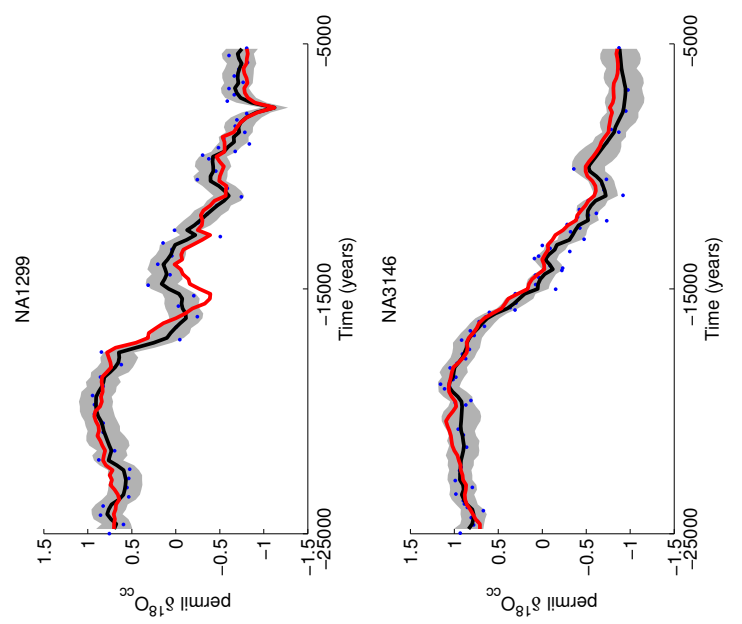

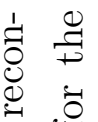

灵

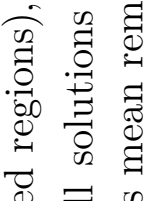

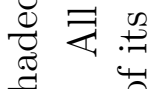

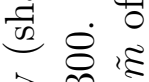

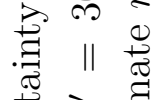

过

$\exists$ 守
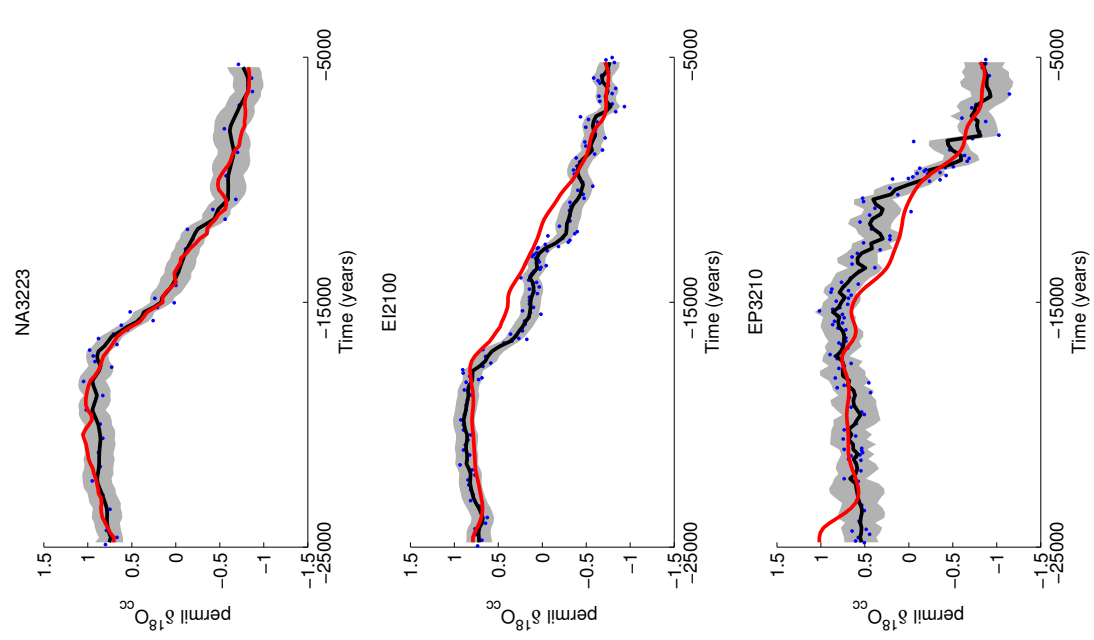

․ㅠ

की

$\exists \stackrel{\Xi}{\Xi}$

궁

를

푸

స 8 శ్

-

过

苛 营

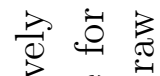

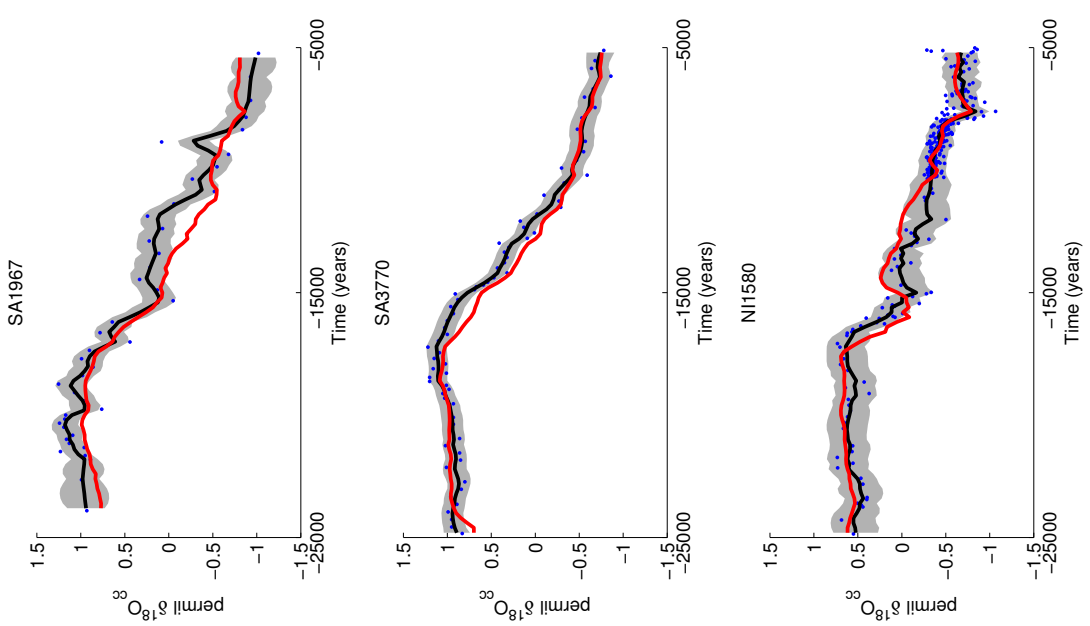

弐

늉

की

앙 है

壱 30

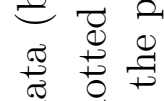

항을

究䨌

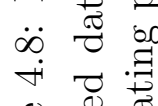

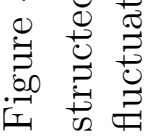



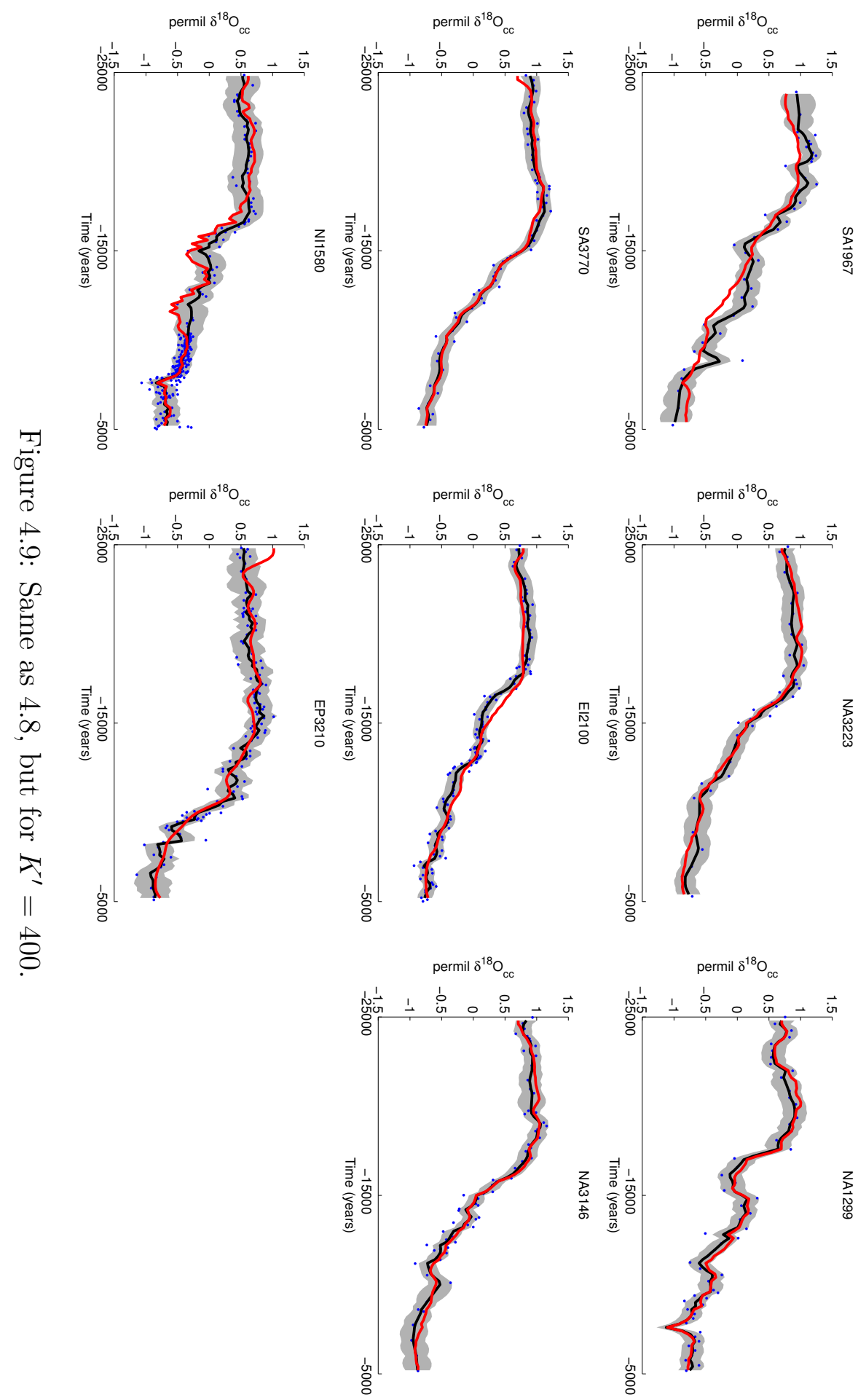
Due to the size of $\mathbf{C}_{\tilde{\mathbf{q}} \tilde{\mathbf{q}}}$, only the diagonal entries $\left[\mathbf{C}_{\tilde{\mathbf{q}} \tilde{\mathbf{q}}}\right]_{i i}$ are considered here. The square root of the diagonal entries is known as the "standard error" and is a measure of the variability of the solution due to uncertainties in the data. Off-diagonal terms contain information about the covariance of the solution. Like the solution $\tilde{\overline{\mathbf{q}}}$, the $\left[\mathbf{C}_{\tilde{\mathbf{q}} \tilde{\mathbf{q}}}\right]_{i i}$ is a three-dimensional field showing how the two-dimensional error changes in time.

Figure 4.10 shows that the spatiotemporal evolution of the $K^{\prime}=400$ solution standard error $\left[\mathbf{C}_{\tilde{\mathbf{q}} \tilde{\mathbf{q}}}\right]_{i i}^{1 / 2}$ is represented well by a single EOF-PC pair. Like the solution EOFs, the uncertainty has nonzero elements in the North Atlantic, Arctic, Ross and Weddell Seas, and southwest of Australia in the Southern Ocean. The PC, which has units of permil $\delta^{18} \mathrm{O}$, holds approximately steady at values between -1.7 and -1.2 for the duration of the reconstruction, which (when scaled by the EOF) suggests an uncertainty of $1 \%$ for many of the locations with nonzero solution amplitudes. The standard error is zero over much of the domain (and at the end of the reconstruction interval, which is ill-constrained by the data) because those regions are not constrained by the data and lie in the solution nullspace. By comparison, the standard error for the $K^{\prime}=300$ case has a similar spatial pattern, but an amplitude reduced by roughly a factor of two, reflecting the lower uncertainty (but larger nullspace) of that solution.

The second component of solution uncertainty, $\mathbf{Q}_{v}\left\langle\boldsymbol{\alpha} \boldsymbol{\alpha}^{\top}\right\rangle \mathbf{Q}_{v}^{\top}$, arises from the expected contributions from the nullspace vectors. By definition, the data are unable to constrain the $\boldsymbol{\alpha}$, and prior notions of these weights must be used if $\mathbf{Q}_{v}\left\langle\boldsymbol{\alpha} \boldsymbol{\alpha}^{\top}\right\rangle \mathbf{Q}_{v}^{\top}$ is to be estimated. The $(2806 * 101) \times(2806 * 101)$ solution resolution matrix $\mathbf{T}_{v}=$ $\mathbf{V}_{K^{\prime}} \mathbf{V}_{K^{\prime}}^{\top}$, where $\mathbf{V}_{K^{\prime}}$ is the $(2806 * 101) \times K^{\prime}$ matrix of solution range vectors, can be useful in understanding which spatiotemporal features lie in the nullspace and in rationalizing differences between true and inferred control values.

For instance, one might ask how a pulse of tracer at -15000 years (i.e., in the 


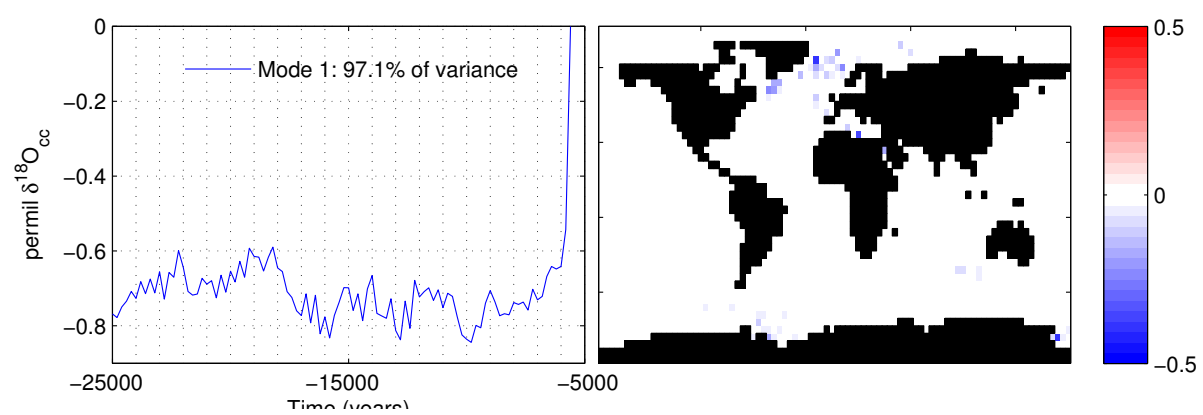

(a)

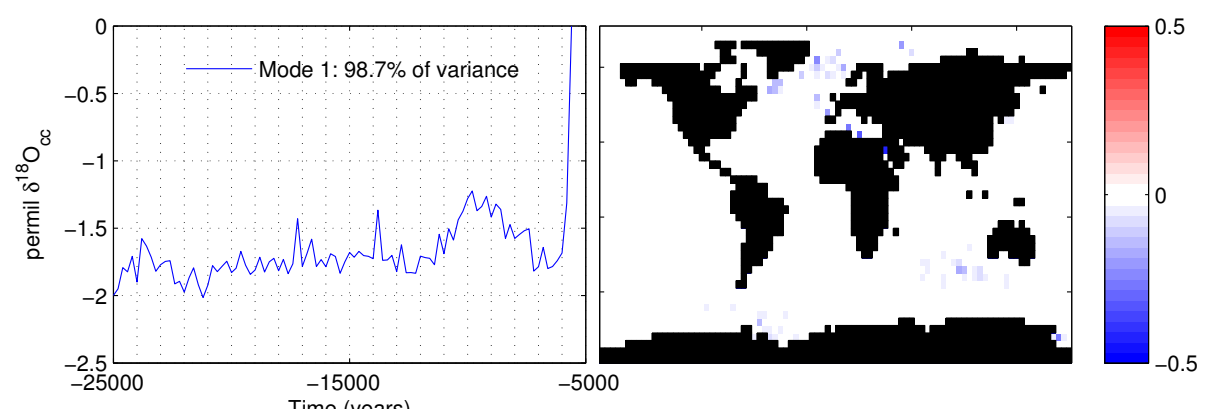

(b)

Figure 4.10: The diagonal elements of the solution covariance matrix $\mathbf{C}_{\tilde{\tilde{\mathbf{q}}} \tilde{\mathbf{q}}}$ (the "standard error") constitute a spatiotemporal pattern with the same dimension as $\tilde{\mathbf{q}}$. Here, the standard error is plotted in terms of its leading EOF-PC pairs for the $K^{\prime}=300$ (4.10a) and $K^{\prime}=400(4.10 \mathrm{~b})$ cases. The EOFs (spatial patterns, right panels) are shown in normalized units, while the PCs (time series, left panels) have the units of the tracer. Times and regions of zero concentration lie in the solution null space of the problem. The leading EOF-PC pair accounts for over $97 \%$ of the variance in both cases and is a good representation of the standard error. 
middle of the reconstruction interval) at a location in the North Atlantic might be inferred by a particular SVD solution with $K^{\prime}=400$. The three panels in Figure 4.11 show maps of the inferred solution $\tilde{\mathbf{q}}$ at three times $(-15200,-15000$, and -14800$)$ when the true control vector $\overline{\mathbf{q}}$ is a Kronecker delta function in time and space, with an amplitude of 1 at the location marked by a green circle (top left) at $t=-15000$ and 0 amplitude at all other places and times. The inferred solution has sidelobes in both time and space. Evidently this choice of control vector did not lie entirely in the space of solution range vectors, i.e., at least some part of the delta function could not be inferred because it lay in the solution null space.

A telling feature of Figure 4.11 is that a delta function of tracer at the location and time chosen is inferred with a $70 \%$ reduction in amplitude (1 vs. 0.3). At other locations, the amplitude reduction is even more dramatic. The inferred amplitude of a delta function in the $i^{t h}$ element of the whole-domain control vector $\overline{\mathbf{q}}$ (i.e., a delta function in time and space) is $\left[\mathbf{T}_{v}\right]_{i i}$. A map of these diagonal elements for the time -15000 is shown in Figure 4.12. At all locations but a few, the variance of the inferred solution at the appropriate place and time is an order of magnitude less than the true value, and in most cases the values are extremely small. One Weddell Sea location is the exception, with a value of 0.94 ; this point is associated with a large ocean volume contribution.

The covariance and resolution matrices are important for interpreting the solution, and evidently several caveats are in order when assessing solutions to the time-varying IBC problem. The solution uncertainty is unbounded over most of the ocean because mixed layer concentrations there lie in the nullspace of the IBC problem. When a component of the controls does not lie entirely in the solution range, the hypothetical solution will have sidelobes in space and time. Thus while the IBC solution (Figure 4.7) appears to be a relatively simple pattern of deglacial change over regions of 

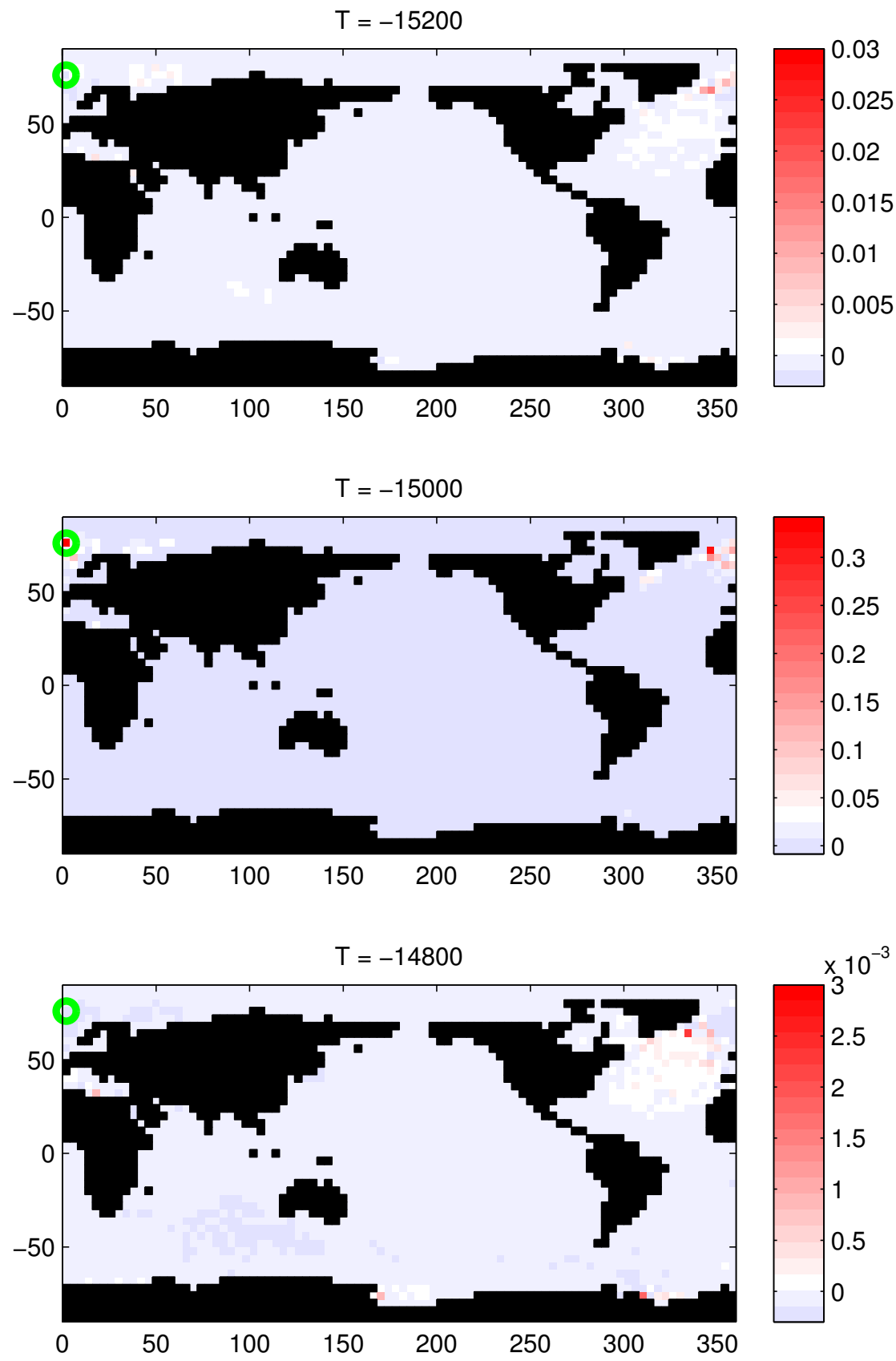

Figure 4.11: Three consecutive columns of the solution resolution matrix $\mathbf{T}_{v}$ show sidelobes in time and space in the inference of a delta function of tracer at the location marked by a green circle (top left) at $t=-15000$. Note that color maps between panels differ by factors of 10 . Rather than reconstructing the delta function, the network of eight sediment cores infers a pattern of nonzero boundary conditions smeared out over the North Atlantic and Arctic Oceans, with a small amount inferred as originating 200 years earlier. 


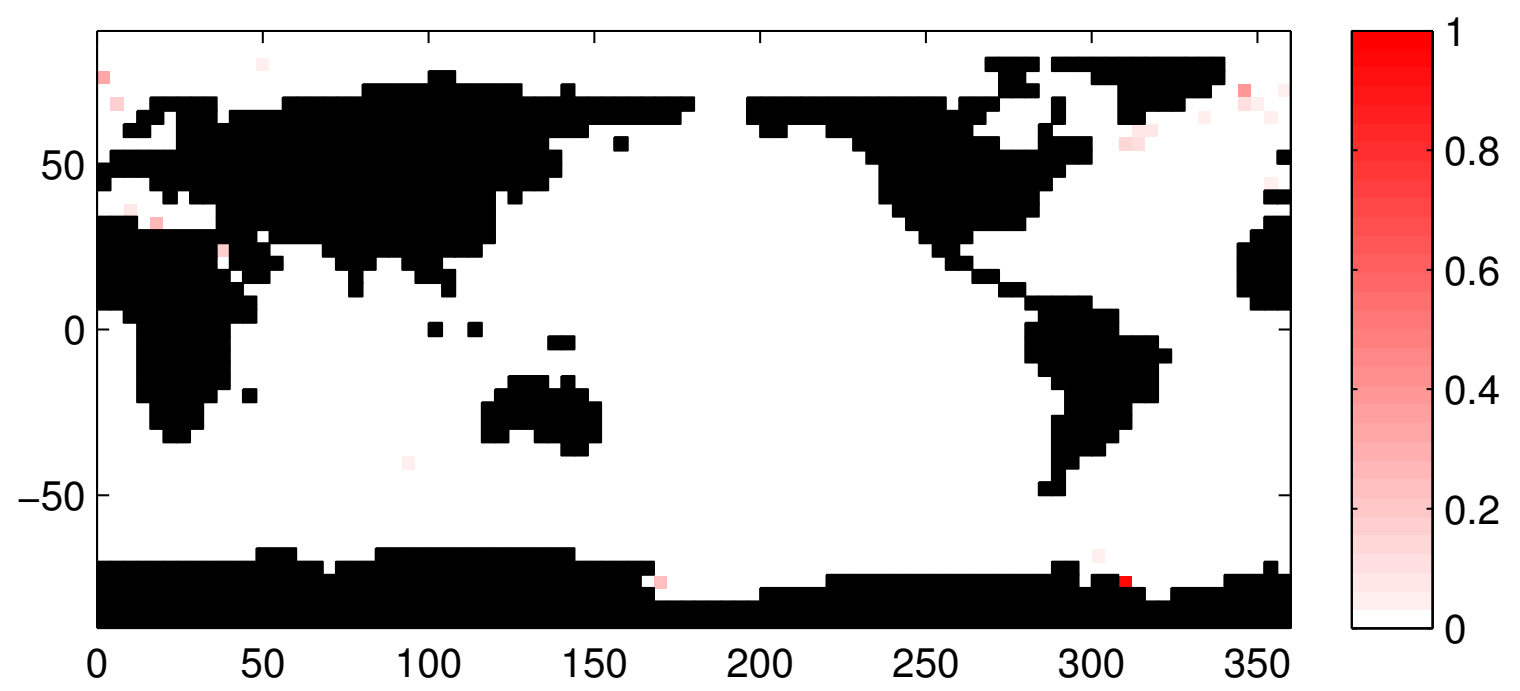

Figure 4.12: Diagonal elements of $\mathbf{T}_{v}$ selected at $t=-15000$ show how variance is attenuated in estimates of surface boundary conditions $\overline{\mathbf{q}}$. For instance, a delta function of tracer released at $t=-15000$ in the Weddell Sea (lower right hand corner, red pixel) will be reconstructed at $t=-15000$ with a value of 0.94 (with additional variance in spatial and temporal sidelobes). For the great majority of surface points, very little or no variance is reconstructed faithfully.

subduction and deepwater formation, with covariance structure that suggests modest uncertainty due to observational error relative to the solution amplitude (Figure 4.10), many features of the "true" solution remain hidden. This observation holds true for any least-squares solution to the IBC problem, not just the SVD solution.

\subsubsection{Data resolution, residuals, and hypothesis testing}

Plots of $\tilde{\mathbf{f}}, \overline{\mathbf{f}}$, and $\overline{\tilde{\mathbf{n}}}$ (Figure 4.9) broadly agree with the interpolated data in many respects. The deglacial $\delta^{18} \mathrm{O}_{c c}$ transition is evident at every core site, and no record-long biases are apparent, though residuals in some records are dominantly low-frequency.

Some sediment core records appear to be better reconstructed than others. The shallower records (NI1580, EI2100, SA1967, and NA1299) appear to share a two- 
stage decrease in $\delta^{18} \mathrm{O}$, with the first transition occurring somewhere between 18 and 16kya; these features are captured in NA1299 and arguably NI1580 but not in the others. By contrast, the deep North Atlantic records (NA3223 and NA3146) show few excursions outside of the 1- $\sigma$ envelope.

The diagonal $\left[\mathbf{T}_{u}\right]_{i i}$ of the $785 \times 785$ data resolution matrix is known as the "data importance" and describes the contributions of an observation in a particular core and time to the corresponding value in the reconstructed data (Figure 4.13). In all cases, data importance is zero at the beginning of the reconstruction interval, a reflection of the effects of initial conditions. The data importance varies by nearly nearly an order of magnitude between some sediment core records. Other variations in $\left[\mathbf{T}_{u}\right]_{i i}$ arise from noise and different row weighting by $\overline{\mathbf{W}}$ reflecting uncertainties from data interpolation. Values of $\left[\mathbf{T}_{u}\right]_{i i}$ corresponding to record SA3770 are nearly 1 over the duration of the record, perhaps because it is the only core site that lies near the Southern Ocean and important deep water formation regions in the Ross and Weddell Seas or perhaps because of its relatively small uncertainty. The data importance of NI1580 appears sensitive to the uncertainty in that record, with several jumps during intervals of high core sampling and commensurate low record uncertainty. Records EI2100, EP3210, and SA1967 have less importance than the other records; evidently more of these records lies in the data nullspace, and their reconstruction would improve with larger choices of $K^{\prime}$.

A visual examination of $\tilde{\overline{\mathbf{f}}}, \overline{\mathbf{f}}$, and $\tilde{\overline{\mathbf{n}}}$ (Figure 4.9 ) shows that residuals are generally larger in some sediment cores than in others and that there are multiple, persistent features in the residuals $\tilde{\overline{\mathbf{n}}}$. Testing the likelihood that $\tilde{\overline{\mathbf{n}}}$ is a realization of the random process described by the prior uncertainty statistics in $\overline{\mathbf{W}}$ is an important step in evaluating whether or not the solution is able to reconstruct the data, and thereby also important in evaluating the hypothesis that the modern circulation can 

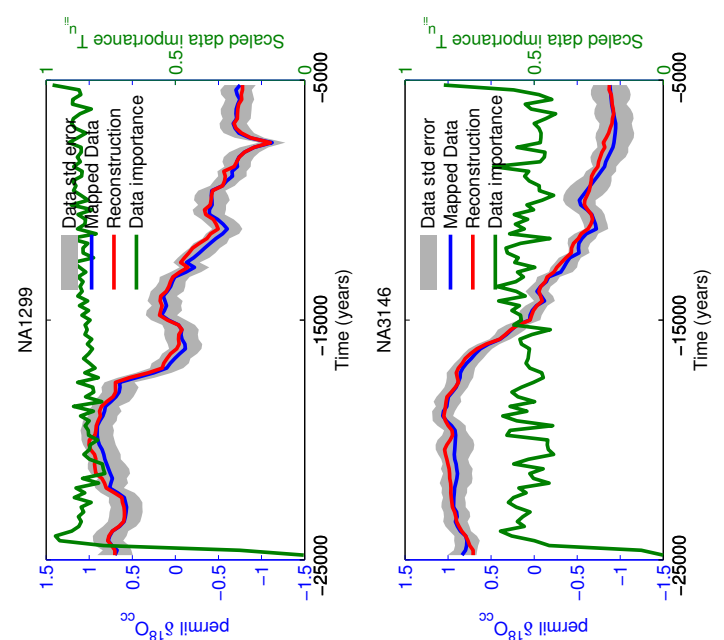

ซี $\mathrm{F}^{*}$

总蒫蒫

"⿻

宽莺

ర్ల

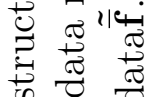

ठํ ठ

$=$

क्ष

.5000

:
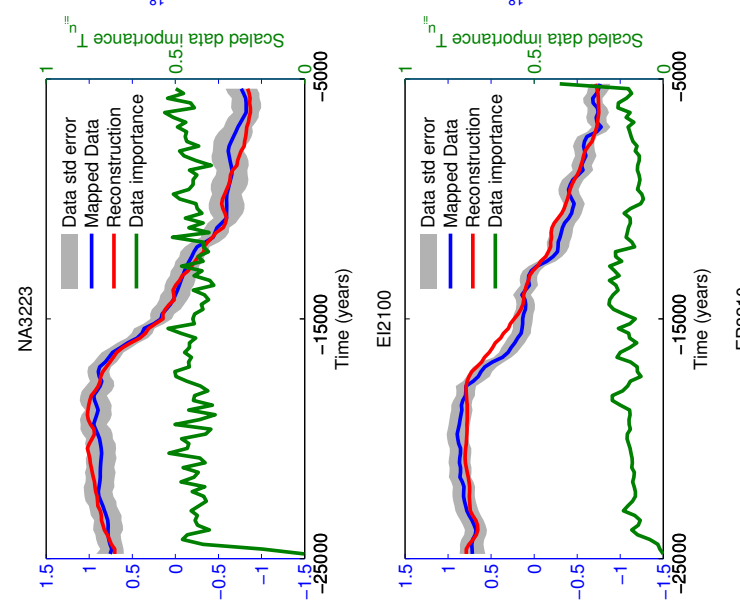

${ }^{20} \mathrm{O}_{81} \mathrm{~s}$ !!wad

${ }^{50} \mathrm{O}_{81} \&$ !!muəd

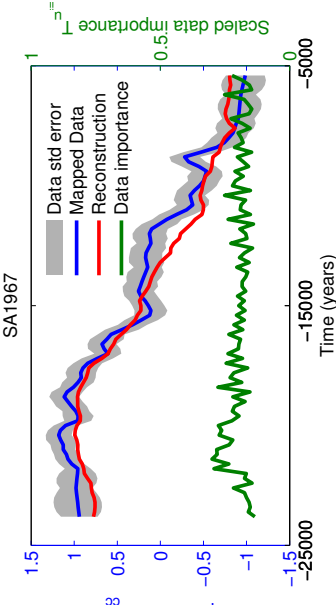

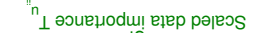
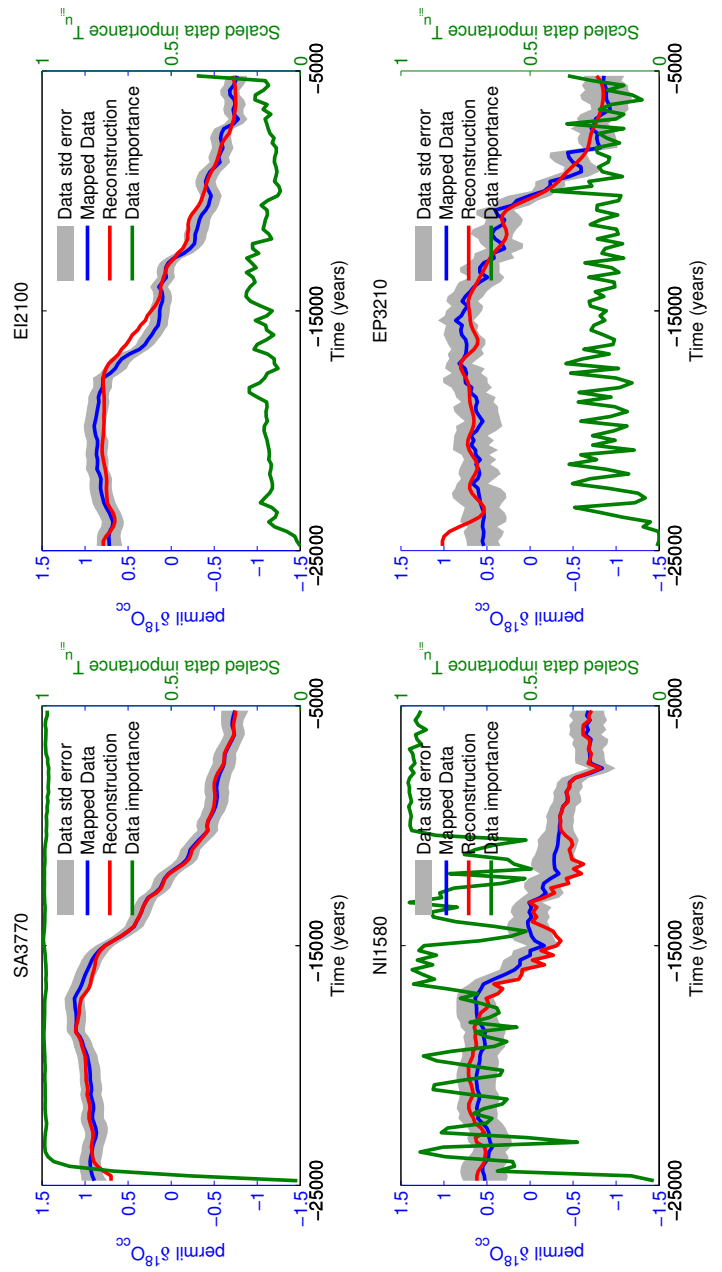

¿

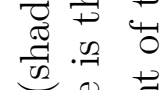

袍

霆

过

尊寻

i劳

की

$\exists$ : 帚

过 80

है

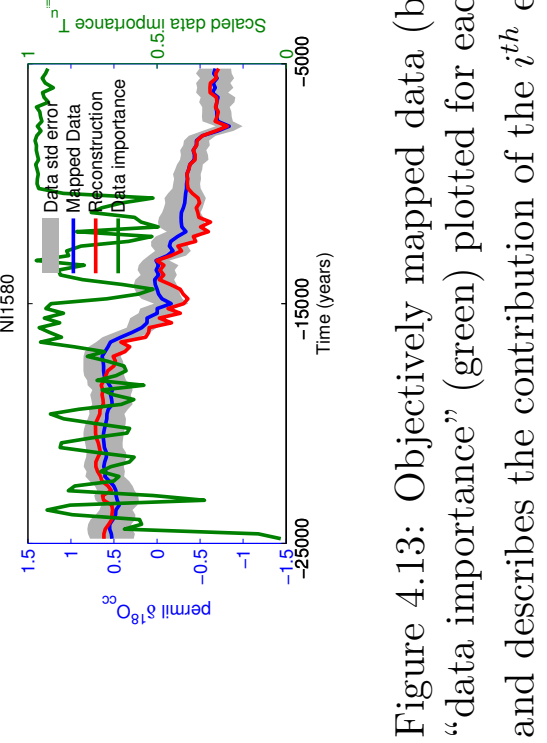




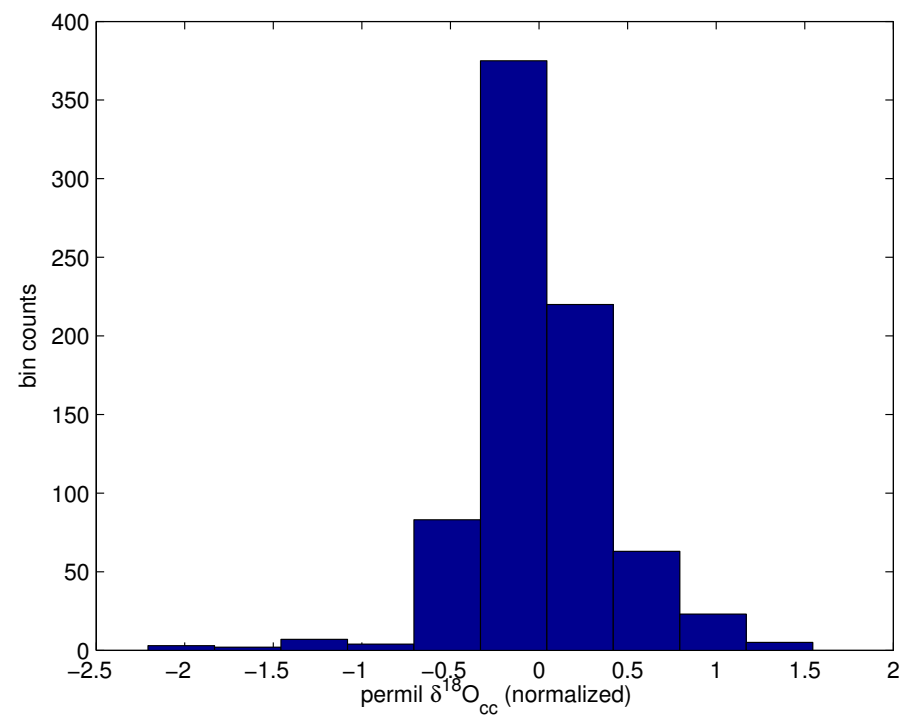

Figure 4.14: Histogram of the scaled residuals $\overline{\mathbf{W}}^{-\top / 2} \tilde{\mathbf{n}}$. The distribution is nonGaussian at the $5 \%$ confidence level.

explain deglacial sediment core records. Ideally, we would like a quantitative way to evaluate whether or not the model-data misfit can be explained by what we think we know about the observational uncertainty.

Here we evaluate only variance and Gaussianity of the scaled residuals $\overline{\mathbf{W}}^{-\top / 2} \tilde{\mathbf{n}}$. The variance of $\overline{\mathbf{W}}^{-\top / 2} \tilde{\mathbf{n}}$ is 0.155 , suggesting that the solution may over-fit the data. A histogram of the scaled residuals is plotted in Figure 4.14. There is a strong peak around 0 and the residuals are skewed right. A $\chi^{2}$ goodness-of-fit test rejects the null hypothesis that the distribution is Gaussian at the $5 \%$ level. The $K^{\prime}=300$ solution has scaled residual variance of 0.32 and is also non-Gaussian.

\subsubsection{A check for self-consistency}

The $\tilde{\mathbf{q}}_{400}$ solution prescribes a set of surface mixed layer concentrations of $\delta^{18} \mathrm{O}_{c c}$, which is related to physical variables through paleotemperature equations, e.g. [Bemis 
et al., 1998]

$$
\delta^{18} O_{c c}=3.4+\delta^{18} O_{s w}-0.21 T .
$$

This section discusses a forward integration of the GH12 model in which the $\tilde{\overline{\mathbf{q}}}_{400}$ mixed layer boundary conditions were applied. Based on the evolution of the tracer field in the model, can we evaluate post hoc whether the passive tracer assumption is tenable?

Figure 4.15 compares the distribution of $\delta^{18} \mathrm{O}_{c c}$ anomaly (varying about the solution mean) in a latitudinal cross-section at $26^{\circ}$ of a snapshot at -15400 years in the forward run to a modern estimate of salinity at the same location from Gouretski and Koltermann [2004]. This time was chosen as an indicative example; $26^{\circ}$ is the location of numerous modern oceanographic surveys and the RAPID array. Noting that modern $\delta^{18} \mathrm{O}_{s w}$-S estimates are 0.51 for NADW 0.23 for AABW [LeGrande and Schmidt, 2006] and assuming that $\delta^{18} \mathrm{O}_{s w}$ accounts for half the variations in $\delta^{18} \mathrm{O}_{c c}$ suggests that if $\delta^{18} \mathrm{O}_{c c}$ were advected as a passive tracer, perturbation zonal salinity gradients in the abyssal ocean from deglacial meltwater would be of the same order as zonal gradients in the modern ocean. Insofar as these gradients affect density, perturbation zonal salinity gradients may induce perturbative meridional flow depending on the relative stratifying effects of temperature on density. Other, different solutions may exist that fit the data but do not imply horizontal salinity gradients. 

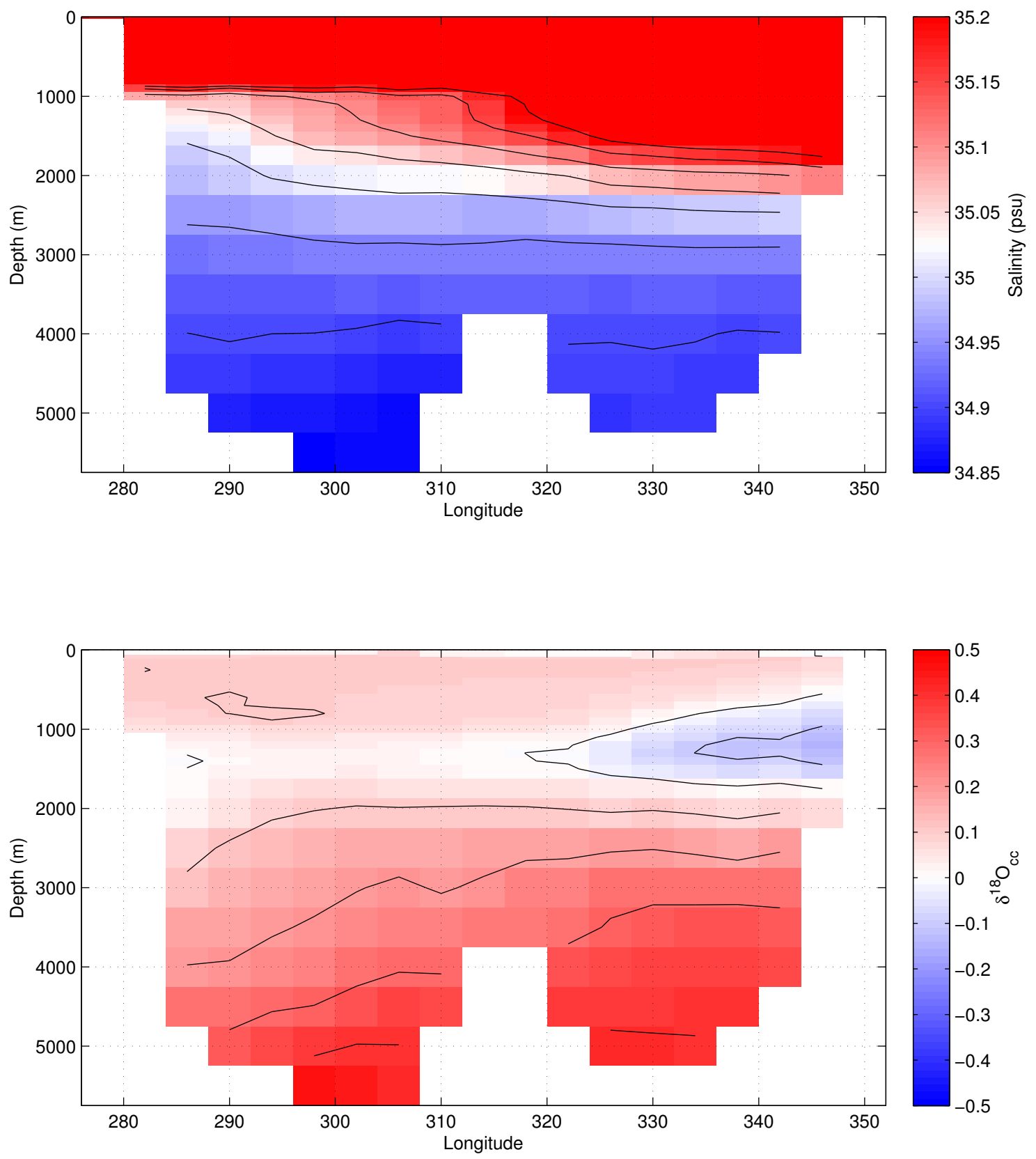

Figure 4.15: TOP: Modern climatological values of salinity in the Atlantic Ocean at $26^{\circ}$ north latitude [Gouretski and Koltermann, 2004]. BOTTOM: Values of $\delta^{18} \mathrm{O}_{c c}$ anomaly at the same location in a forward integration of the GH12 tracer transport model forced by controls derived in the inverse problem with $K^{\prime}=400$. 


\subsection{Discussion}

This chapter posed a tracer inverse boundary condition problem in the context of deglacial benthic $\delta^{18} \mathrm{O}$ records of calcium carbonate recovered at eight core sites. Particular SVD solutions for the time history of surface mixed layer boundary conditions were derived for the (mean-removed) sediment core records and for the average values of the records on the interval [-25000,20000] (representative of the LGM). A time-invariant set of boundary conditions was found that satisfied the LGM-era means.

The characteristics of the time-varying solution, and the corresponding fit to the data, changes substantially with different choices of the SVD truncation parameter $K^{\prime}$. Lower values of this parameter yield solutions with magnitudes of $\delta^{18} \mathrm{O}_{c c}$ more like those found in today's ocean, but at the expense of increased misfit with the data.

The formal disagreement of the solution residuals with their a priori statistics says that something is wrong with the assumptions that went into the inverse model - the way the data were processed, the representation of sediment core error, the ability of GH12 to represent the modern ocean, or the ability of the modern ocean to explain deglacial sediment core records. In this case, the skewness of the residuals appears to be due to misfits with two cores (SA1967 and NI1580) over several contiguous intervals, and the non-Gaussianity of the residuals could be removed if the conservative error estimate of $0.2 \%$ used in the construction of sediment core records were reduced. Given the great uncertainty surrounding error estimates of sediment core records, there is insufficient evidence to demonstrate that the modern circulation did not persist throughout the deglaciation without the inclusion of additional information. Further investigation of the misfits of SA1967 and NI1580 is a clear avenue 


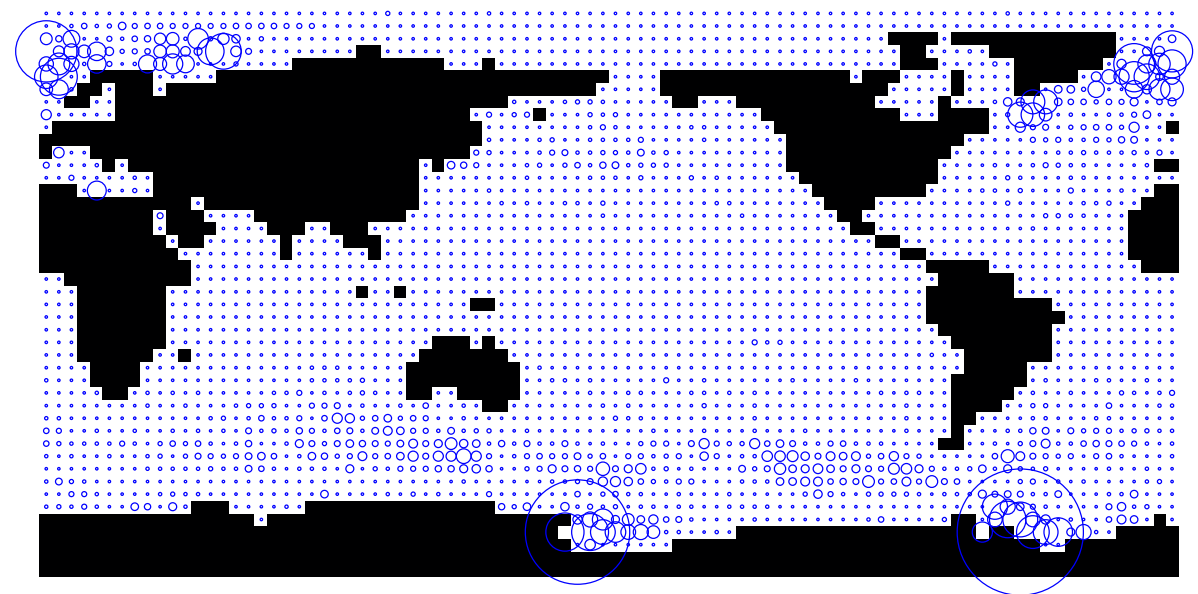

Figure 4.16: The global ocean volume is filled disproportionately in the steady pathways model of Gebbie and Huybers [2010] by a subset of surface grid boxes. In this figure, circle radii are propotional to the proportion of ocean volume below the mixed layer that originates from the corresponding surface locations. Figure 2 from Gebbie and Huybers [2011] is a logarithmic version of this plot.

for future work.

Another insight is that solutions obtained using SVD (without imposing prior information about solutions) have spatial structures that are determined primarily by the resolving power of the network of benthic records. Comparisons of the spatial structure in the solutions or uncertainties with Figure 4.16, which shows the contributions of surface locations to the global ocean volume in the model of GH10, demonstrates that solutions have positive variance in regions that are most responsible for filling the ocean. The algebraic understanding comes from the resolution matrix: Range vectors of the IBC problem have spatial structure derived from the distribution of dominant deepwater locations. A simple analog is provided in the 4box model in Section 2.4.2 and discussed in Section 2.4.4. The physical understanding is straightforward: measurements of scalar properties in the deep ocean only constrain 
properties of parcels that actually enter the deep ocean.

The solutions, dominated as they are by the resolvable spatial patterns of the problem, do not resemble the much smoother distributions of $\theta$ and $\delta^{18} \mathrm{O}_{s w}$ that are expected for physical reasons and observed in the modern ocean. Moreover, some inferred surface tracer concentrations have much larger amplitudes than observed in the modern [LeGrande and Schmidt, 2006, Gouretski and Koltermann, 2004] and are negative. Given these a priori unreasonable solution characteristics, physical interpretations of the solutions should be approached cautiously; however, several features in the time-variability of the solutions are suggestive of canonical stages of last deglacial climate (reflecting similar variability in the sediment core records).

From the unphysicality of the solution, one is led to conclude that the SVD solution approach is inappropriate in this context to test the null hypothesis that a solution $\tilde{\overline{\mathbf{q}}}$ can be found that fits the benthic observations using an estimate of the modern circulation. A solution can be found, but clearly other information must be brought to bear in the problem to find a solution that fits a priori notions of physicality. One way forward is the use of column weighting as in Gebbie [2012]. Despite this drawback, the SVD approach has proved insightful for understanding and quantifying the nullspaces of the problem. 


\section{Chapter 5}

\section{Concluding remarks}

This thesis develops and applies a framework for understanding deglacial benthic records of $\delta^{18} \mathrm{O}_{c c}$ in the context of the tracer inverse problem, the goal of which is to infer boundary conditions and flow parameters from observations of a fluid system (the ocean). The approach taken is to solve the inverse boundary problem (IBC), whereby a time history of surface mixed layer $\delta^{18} \mathrm{O}_{c c}$ concentrations is sought that, when propagated in space using an estimate of the modern mass circulation, best agrees with the data in a least-squares sense.

Some of the lessons learned in the ocean inverse problem can be understood in the context of the much simpler box models studied in Chapter 2. Advective-diffusive properties delay and smooth tracer signals that are imposed at system boundaries, and the effect of tracer signal propagation in steady flow is mathematically the same as the application of a linear, time-invariant filter, as also discussed in Rutberg and Peacock [2006]. The solution nullspace is the set of features that cannot be inferred from the data. Nullspaces in the inverse tracer problem can arise from observational sparsity, attenuation of structures by tracer transport (e.g. mixing), and vanishing 
influence of the controls on the system state.

In Chapter 3, eight sediment core records were discussed and processed for use in the IBC problem. Objective mapping was used to interpolate the records to be evenly spaced in time and to estimate uncertainty in the data due to uneven sampling. This procedure inferred higher uncertainties during times of sparse sampling and tended to smooth over rapid transitions or spikes.

Chapter 4 solves the IBC problem for the evolution of $\delta^{18} \mathrm{O}_{c c}$ in the ocean mixed layer under the assumption that the modern ocean circulation, as estimated by Gebbie and Huybers [2010, 2012], persisted over the time interval [-25000,-5000]. Particular SVD solutions for the time history of surface mixed layer $\delta^{18} \mathrm{O}_{c c}$ were derived, constrained by the eight sediment core records discussed in Chapter 3. The inverse problem seeks to infer $\delta^{18} \mathrm{O}_{c c}$ at 2806 locations and is highly underdetermined.

The inverse problem has a large nullspace, and the data can be accommodated reasonably well in the fluctuating problem using the 400 leading singular vectors. A solution obtained using 300 leading singular vectors had lower amplitude and uncertainty but did not resolve the Atlantic-Pacific lag introduced in Chapter 1. Solutions are dominated by resolvable patterns of the data, which reflect locations and magnitudes of ocean volume contributions. There are time-persistent features in the residuals of two records, despite the fact that the total variance of the scaled residuals suggests that the solution over-fits the data.

Given the large uncertainties in sediment core error models and the unphysicality of the solutions, we conclude the records cannot disprove the null hypothesis that the modern ocean circulation persisted during the deglaciation. Solutions obtained (e.g. Gebbie [2012]) that explicitly use information about surface tracer distributions are more appropriate for testing the null hypothesis that the modern circulation persisted during the deglaciation. 


\section{Directions for future work}

Is $\delta^{18} \mathbf{O}_{c c}$ a passive tracer? The deglacial freshwater forcing problem is challenging and alluring because it involves most of the processes studied in physical oceanography. The beginning of the $\delta^{18} \mathrm{O}_{c c}$ transport pathway begins when cold, fresh water is released into the coastal ocean (except in the case of Heinrich events, where a substantial amount of ice is thought to melt in the open ocean), probably forming a buoyant coastal plume that is eventually entrained into gyre-scale flow [Condron and Winsor, 2011]. The $\delta^{18} \mathrm{O}_{c c}$ signal will be transmitted to the abyssal ocean via subduction or convective deep water formation. Buoyancy anomalies in the deep ocean can lead to the reorganization of baroclinic structure through the transmission of planetary waves [Kawase, 1987]. A major question in implicating freshwater forcing in past ocean circulation changes is to what extent $\delta^{18} \mathrm{O}_{c c}$ may be treated as a passive tracer; Siberlin [2010] provides some first steps in a modeling framework.

What kinds of changes can be inferred by available observations? The detectability of circulation changes by available data should be weighed when evaluating any hypotheses about past ocean evolution. There is naturally a nullspace for detecting changes in flow parameters, e.g. if a change in circulation $\Delta \mathbf{G}$ obeys

$$
\overline{\mathbf{B}}(\overline{\mathbf{G}}+\Delta \overline{\mathbf{G}}) \overline{\mathbf{F}} \overline{\mathbf{q}}=\mathbf{0}
$$

then it is unobservable. Clearly the form of $\overline{\mathbf{q}}$ is important in governing observability, motivating the study of cases (such as the deglaciation) with relatively well-defined boundary conditions.

Construct an improved error model. Problems with data - sparsity, covari- 
ance, and noise - are endemic in paleoceanography. In this thesis, effort was taken to treat records uniformly and consistently, but the statistics of sediment core errors - including human error, undersea landslides, instrument drift, age model error, and other potentially non-Gaussian processes that are difficult to quantify - may be so complicated that only an expert can actually say what is going on in a particular record. Unless such an expert is willing to assign uncertainties to individual data points in a sediment core, this leaves inverse studies with an insoluble quandary of ad hoc error statistics. Alternatively, a concerted effort to simulate, via pseudoproxy studies or otherwise, and characterize the uncertainty in sediment core records may make such records better suited for use in quantitative problems.

Where should new records be recovered? Locations and times that reduce the null space are desirable for future sediment core recovery in order to better constrain the IBC problem.

Incorporate new benthic $\delta^{18} \mathrm{O}$ records from New Zealand. A yet-unpublished set of deglacial $\delta^{18} \mathrm{O}_{c c}$ records (E. Sikes, pers. comm.) was recovered at multiple depths in the water column and dated using ash layers. The cores' proximity to modern regions of deepwater formation in the GH12 representation of the Southern Ocean is ideally suited to reducing the nullspace of the IBC problem.

Assemble a network of planktonic deglacial $\delta^{18} \mathrm{O}$ records in regions of deepwater formation. These records can be compared to the inverse solutions obtained here or included explicitly in the inverse problem to test the consistency of the modern circulation with both benthic and planktonic tracer observations. If the data are inconsistent with a steady modern flow assumption, can they be used to constrain aspects of the flow evolution? A first assumption is that modern and LGM states form a basis for the intervening times (i.e. that tracer transport in the deglaciation is a weighted sum of the modern and LGM transports). 
Test the sensitivity of conclusions to age model errors. How large must reservoir age errors be in order to explain phase offsets between benthic $\delta^{18} \mathrm{O}$ records?

Derive Green functions from other state estimates. How sensitive are the results in this thesis to the choice of circulation estimate? What does the inverse problem look like in circulations representative of other climates? Are some inferences about surface climate conditions (via boundary conditions) robust to differences in circulation estimates? The Holocene and LGM bookend the deglaciation, and the deglacial IBC problem could equally well be solved with an LGM tracer transport estimate.

Express tracer inverse problems in terms of concentrations exported from the mixed layer. Given that reconstruction variance is focused on deepwater formation regions in the model of GH12, it seems likely that inferences of mean tracer concentrations exported from the ML in a geographical region can be inferred much more robustly than the average ML tracer concentrations there. The rationale for this result echoes a recurring theme in this thesis: benthic observations can only constrain properties that are exported to the deep ocean. 


\section{Bibliography}

R.B. Alley, J. Marotzke, WD Nordhaus, JT Overpeck, DM Peteet, RA Pielke Jr, RT Pierrehumbert, PB Rhines, TF Stocker, LD Talley, et al. Abrupt climate change. Science, 299(5615):2005-2010, 2003.

R.B. Alley, S. Anandakrishnan, T.K. Dupont, B.R. Parizek, and D. Pollard. Effect of sedimentation on ice-sheet grounding-line stability. Science, 315(5820):1838-1841, 2007.

DC Barber, A Dyke, C Hillaire-Marcel, AE Jennings, JT Andrews, MW Kerwin, G Bilodeau, R McNeely, J Southon, MD Morehead, et al. Forcing of the cold event of 8,200 years ago by catastrophic drainage of Laurentide lakes. Nature, 400(6742): 344-348, 1999.

E. Bard, M. Arnold, B. Hamelin, N. Tisnerat-Laborde, and G. Cabioch. Radiocarbon calibration by means of mass spectrometric $230 \mathrm{Th} / 234 \mathrm{U}$ and $14 \mathrm{C}$ ages of corals: an updated database including samples from Barbados, Mururoa and Tahiti. Radiocarbon, 40(3):1085-1092, 1998.

B.E. Bemis, H.J. Spero, J. Bijma, and D.W. Lea. Reevaluation of the oxygen isotopic composition of planktonic foraminifera: Experimental results and revised paleotemperature equations. Paleoceanography, 13(2):150-160, 1998.

M. Blaauw, G. Heuvelink, D. Mauquoy, J. van der Plicht, and B. van Geel. A numerical approach to $14 \mathrm{C}$ wiggle-match dating of organic deposits: best fits and confidence intervals. Quaternary science reviews, 22(14):1485-1500, 2003. ISSN 0277-3791.

T. Blunier and E.J. Brook. Timing of millennial-scale climate change in Antarctica and Greenland during the last glacial period. Science, 291(5501):109, 2001.

LI Bradtmiller, RF Anderson, MQ Fleisher, and LH Burckle. Opal burial in the equatorial Atlantic Ocean over the last $30 \mathrm{ka}$ : Implications for glacial-interglacial changes in the ocean silicon cycle. Paleoceanography, 22(4):PA4216, 2007. 
Christopher S Bretherton, Catherine Smith, and John M Wallace. An intercomparison of methods for finding coupled patterns in climate data. Journal of climate, 5(6): 541-560, 1992 .

Wallace S Broecker. Paleocean circulation during the last deglaciation: a bipolar seesaw? Paleoceanography, 13(2):119-121, 1998.

Wallace S Broecker, Sean Blanton, William M Smethie Jr, and Gote Ostlund. Radiocarbon decay and oxygen utilization in the deep Atlantic Ocean. Global Biogeochemical Cycles, 5(1):87-117, 1991.

W.S. Broecker. Does the trigger for abrupt climate change reside in the ocean or in the atmosphere? Science, 300(5625):1519-1522, 2003.

C. Bronk Ramsey. Bayesian analysis of radiocarbon dates. Radiocarbon, 51(1):337360, 2009.

Harry L Bryden. New polynomials for thermal expansion, adiabatic temperature gradient and potential temperature of sea water. In Deep Sea Research and Oceanographic Abstracts, volume 20, pages 401-408. Elsevier, 1973.

CE Buck. Bayesian Chronological Data Interpretation: Where Now? LECTURE NOTES IN STATISTICS-NEW YORK-SPRINGER VERLAG-, pages 1-24, 2004. ISSN 0930-0325.

C.E. Buck and A. Millard. Tools for constructing chronologies: crossing disciplinary boundaries. Springer Verlag, 2004. ISBN 185233763X.

Andrea Burke, Olivier Marchal, Louisa I Bradtmiller, Jerry F McManus, and Roger François. Application of an inverse method to interpret 231Pa/230Th observations from marine sediments. Paleoceanography, 26(1), 2011.

A. Condron and P. Winsor. A subtropical fate awaited freshwater discharged from glacial Lake Agassiz. Geophysical Research Letters, 38(3):L03705, 2011.

T.M. Cronin. Paleoclimates: understanding climate change past and present. Columbia University Press, 2010.

W.B. Curry and D.W. Oppo. Glacial water mass geometry and the distribution of d13C of sigmaCO2 in the western Atlantic Ocean. Paleoceanography, 20(1):PA1017, 2005.

W.B. Curry, J.C. Duplessy, L.D. Labeyrie, and N.J. Shackleton. Changes in the distribution of $\mathrm{d} 13 \mathrm{C}$ of deep water sigmaCO2 between the last glaciation and the Holocene. Paleoceanography, 3(3):317-341, 1988. 
Holly Dail. Atlantic Ocean circulation at the Last Glacial Maximum: Inferences from data and models. PhD thesis, MIT-WHOI Joint Program, 2012.

Willi Dansgaard, SJ Johnsen, HB Clausen, D Dahl-Jensen, NS Gundestrup, CU Hammer, CS Hvidberg, JP Steffensen, AE Sveinbjörnsdottir, J Jouzel, et al. Evidence for general instability of past climate from a 250-kyr ice-core record. Nature, 364 (6434):218-220, 1993.

I. Eisenman, C.M. Bitz, and E. Tziperman. Rain driven by receding ice sheets as a cause of past climate change. Paleoceanography, 24(4):PA4209, 2009.

R.G. Fairbanks, R.A. Mortlock, T.C. Chiu, L. Cao, A. Kaplan, T.P. Guilderson, T.W. Fairbanks, A.L. Bloom, P.M. Grootes, and M.J. Nadeau. Radiocarbon calibration curve spanning 0 to 50,000 years BP based on pairedi supi 230i/supi Th/i supi 234i/supi U/i supi 238i/supi U andi supi $14 i / \sup i \mathrm{C}$ dates on pristine corals. Quaternary Science Reviews, 24(16):1781-1796, 2005.

Nick P Fofonoff and Robert C Millard. Algorithms for computation of fundamental properties of seawater. 1983.

Claude Frankignoul and Klaus Hasselmann. Stochastic climate models, part II application to sea-surface temperature anomalies and thermocline variability. Tellus, 29(4):289-305, 1977.

Gene F Franklin, Michael L Workman, and Dave Powell. Digital control of dynamic systems. Addison-Wesley Longman Publishing Co., Inc., 1997.

T. Friedrich and A. Timmermann. Millennial-scale glacial meltwater pulses and their effect on the spatiotemporal benthic $\delta 18 \mathrm{O}$ variability. Paleoceanography, 27(3): PA3215, 2012.

G. Gebbie. Tracer transport timescales and the observed Atlantic-Pacific lag in the timing of the Last Termination. Paleoceanography, 27(3):PA3225, 2012.

G. Gebbie and P. Huybers. Total matrix intercomparison: a method for resolving the geometry of water-mass pathways. J. Phys. Oceanogr, 40(8):1710-1728, 2010.

G. Gebbie and P. Huybers. How is the ocean filled? Geophysical Research Letters, 38(6):L06604, 2011.

G. Gebbie and P. Huybers. The mean age of ocean waters inferred from radiocarbon observations: sensitivity to surface sources and accounting for mixing histories. Journal of Physical Oceanography, 42(2):291-305, 2012.

Davide Giraudo. Connection between Sum of Columns in Matrix and largest eigenvalues. Mathematics Stack Exchange. URL http://math. stackexchange.com/q/ 202937. URL:http://math.stackexchange.com/q/202937 (version: 2012-09-26). 
Gene H Golub and Charles F Van Loan. Matrix computations, volume 3. JHU Press, 2012.

N. Gomez, J.X. Mitrovica, P. Huybers, and P.U. Clark. Sea level as a stabilizing factor for marine-ice-sheet grounding lines. Nature Geoscience, 3(12):850-853, 2010.

Viktor Gouretski and Klaus Peter Koltermann. WOCE global hydrographic climatology. Berichte des BSH, 35:1-52, 2004.

P.M. Grootes, M. Stuiver, JWC White, S. Johnsen, and J. Jouzel. Comparison of oxygen isotope records from the GISP2 and GRIP Greenland ice cores. 1993.

Eddie Haam and Peter Huybers. A test for the presence of covariance between timeuncertain series of data with application to the Dongge Cave speleothem and atmospheric radiocarbon records. Paleoceanography, 25(2), 2010.

JD Hays, J. Imbrie, and NJ Shackleton. Variations in the Earth's Orbit: Pacemaker of the Ice Ages. Science, 194:1121-1134, 1976.

Mark Holzer and François W Primeau. Improved constraints on transit time distributions from argon 39: A maximum entropy approach. Journal of Geophysical Research: Oceans (1978-2012), 115(C12), 2010.

Konrad A Hughen, John R Southon, Scott J Lehman, and Jonathan T Overpeck. Synchronous radiocarbon and climate shifts during the last deglaciation. Science, 290(5498):1951-1954, 2000.

Konrad A Hughen, Mike GL Baillie, Edouard Bard, J Warren Beck, Chanda JH Bertrand, Paul G Blackwell, Caitlin E Buck, George S Burr, Kirsten B Cutler, Paul E Damon, et al. Marine04 marine radiocarbon age calibration, 0-26 cal kyr BP. 2004.

M. Hüls and R. Zahn. Millennial-scale sea surface temperature variability in the western tropical North Atlantic from planktonic foraminiferal census counts. Paleoceanography, 15(6):659-678, 2000.

P. Huybers and C. Wunsch. A depth-derived Pleistocene age model: Uncertainty estimates, sedimentation variability, and nonlinear climate change. Paleoceanography, 19(1):PA1028, 2004. ISSN 0883-8305.

P. Huybers and C. Wunsch. Obliquity pacing of the late Pleistocene glacial terminations. Nature, 434(7032):491-494, 2005.

Peter Huybers and William Curry. Links between annual, Milankovitch and continuum temperature variability. Nature, 441(7091):329-332, 2006. 
Peter Huybers and Carl Wunsch. Paleophysical oceanography with an emphasis on transport rates. Marine Science, 2, 2010.

Peter Huybers, Geoffrey Gebbie, and Olivier Marchal. Can paleoceanographic tracers constrain meridional circulation rates? Journal of physical oceanography, 37(2): 394-407, 2007.

J. Imbrie, JD Hays, DG Martinson, A. McIntyre, AC Mix, JJ Morley, NG Pisias, WL Prell, and NJ Shackleton. The orbital theory of Pleistocene climate: Support from a revised chronology of the marine delta18 $\mathrm{O}$ record. In Milankovitch and climate: Understanding the response to astronomical forcing, volume 1, page 269, 1984.

T.S. Ivanochko, R.S. Ganeshram, G.J.A. Brummer, G. Ganssen, S.J.A. Jung, S.G. Moreton, and D. Kroon. Variations in tropical convection as an amplifier of global climate change at the millennial scale. Earth and Planetary Science Letters, 235 (1-2):302-314, 2005.

Gregory C Johnson. Quantifying Antarctic bottom water and North Atlantic deep water volumes. Journal of Geophysical Research: Oceans (1978-2012), 113(C5), 2008.

I. Joughin, I.M. Howat, M. Fahnestock, B. Smith, W. Krabill, R.B. Alley, H. Stern, and M. Truffer. Continued evolution of Jakobshavn Isbrae following its rapid speedup. J. geophys. Res, 113(F04006):F04006, 2008.

I. Joughin, R.B. Alley, and D.M. Holland. Ice-Sheet Response to Oceanic Forcing. Science, 338(6111):1172-1176, 2012.

SJA Jung, GM Ganssen, and GR Davies. Multidecadal variations in the early Holocene outflow of Red Sea Water into the Arabian Sea. Paleoceanography, 16 (6):658-668, 2001.

S.J.A. Jung, D. Kroon, G. Ganssen, F. Peeters, and R. Ganeshram. Enhanced Arabian Sea intermediate water flow during glacial North Atlantic cold phases. Earth and Planetary Science Letters, 280(1-4):220-228, 2009.

Mitsuhiro Kawase. Establishment of deep ocean circulation driven by deep-water production. Journal of Physical Oceanography, 17(12):2294-2317, 1987.

LD Keigwin and GA Jones. Western North Atlantic evidence for millennial-scale changes in ocean circulation and climate. Journal of Geophysical Research, 99(C6): 12397-12, 1994.

LD Keigwin and MA Schlegel. Ocean ventilation and sedimentation since the glacial maximum at $3 \mathrm{~km}$ in the western North Atlantic. Geochemistry, Geophysics, Geosystems, 3(6):1-14, 2002. 
LD Keigwin, GA Jones, SJ Lehman, and EA Boyle. Deglacial meltwater discharge, North Atlantic deep circulation, and abrupt climate change. Journal of Geophysical Research, 96(C9):16811-16, 1991.

Robert M Key, Alex Kozyr, Chris L Sabine, Kitack Lee, Rik Wanninkhof, John L Bullister, Richard A Feely, Frank J Millero, Calvin Mordy, and T-H Peng. A global ocean carbon climatology: Results from Global Data Analysis Project (GLODAP). Global Biogeochemical Cycles, 18(4), 2004.

John A Knauss. Introduction to physical oceanography. 1996.

PM Kroopnick. The distribution of $i \sup _{i} 13_{i} / \sup _{i}$ C of SsigmaCO $i$ subi $2 i / \operatorname{sub}_{i}$ in the world oceans. Deep Sea Research Part A. Oceanographic Research Papers, 32 (1):57-84, 1985 .

D.W. Lea, D.K. Pak, L.C. Peterson, and K.A. Hughen. Synchroneity of tropical and high-latitude Atlantic temperatures over the last glacial termination. Science, 301 (5638):1361-1364, 2003.

P. LeGrand and C. Wunsch. Constraints from paleotracer data on the North Atlantic circulation during the last glacial maximum. Paleoceanography, 10(6):1011-1045, 1995.

A.N. LeGrande and G.A. Schmidt. Global gridded data set of the oxygen isotopic composition in seawater. Geophysical Research Letters, 33(12):L12604, 2006.

J. Lippold, J.M. Gherardi, and Y. Luo. Testing the 231Pa/230Th paleocirculation proxy: A data versus 2D model comparison. Geophysical Research Letters, 38(20): L20603, 2011.

L.E. Lisiecki and M.E. Raymo. A Plio-Pleistocene stack of 57 globally distributed benthic $\delta 180$ records. Paleoceanography, 20(1), 2005.

M. Little, RR. Schneider, D. Kroon, T. Price, T. Bickert, and G. Weger. Rapid palaeoceanographic changes in the Benguela Upwelling System for the last 160,000 years as indicated by abundances of planktonic foraminifer. Paleogeography, Palaeoclimatology, Palaeoecology, 130:135-161, 1997a.

M.G. Little, R.R. Schneider, D. Kroon, B. Price, C.P. Summerhayes, and M. Segl. Trade wind forcing of upwelling, seasonality, and Heinrich events as a response to sub-Milankovitch climate variability. Paleoceanography, 12:568-576, 1997b.

DC Lund, JF Adkins, and R. Ferrari. Abyssal Atlantic circulation during the Last Glacial Maximum: Constraining the ratio between transport and vertical mixing. Paleoceanography, 26(1):PA1213, 2011. 
J. Lynch-Stieglitz, W.B. Curry, and N. Slowey. Weaker Gulf Stream in the Florida straits during the last glacial maximum. Nature, 402(6762):644-648, 1999.

S. Manabe and RJ Stouffer. Two stable equilibria of a coupled ocean-atmosphere model. Journal of Climate, 1(9):841-866, 1988.

Olivier Marchal and William B Curry. On the abyssal circulation in the glacial Atlantic. Journal of Physical Oceanography, 38(9):2014-2037, 2008.

John C Marshall, Richard G Williams, and AJ George Nurser. Inferring the subduction rate and period over the North Atlantic. Journal of Physical Oceanography, 23(7):1315-1329, 1993.

JF McManus, R. Francois, J.M. Gherardi, LD Keigwin, and S. Brown-Leger. Collapse and rapid resumption of Atlantic meridional circulation linked to deglacial climate changes. Nature, 428(6985):834-837, 2004.

DR Ostermann and WB Curry. Calibration of stable isotopic data: an enriched $\delta 180$ standard used for source gas mixing detection and correction. Paleoceanography, $15(3): 353-360,2000$.

S. Peacock and M. Maltrud. Transit-time distributions in a global ocean model. Journal of Physical Oceanography, 36(3):474-495, 2006.

WR Peltier and RG Fairbanks. Global glacial ice volume and Last Glacial Maximum duration from an extended Barbados sea level record. Quaternary Science Reviews, 25(23):3322-3337, 2006.

William H Press, George B Rybicki, and Jacqueline N Hewitt. The time delay of gravitational lens 0957+ 561. I-Methodology and analysis of optical photometric data. II-Analysis of radio data and combined optical-radio analysis. The Astrophysical Journal, 385:404-420, 1992.

F. Primeau, E. Deleersnijder, et al. On the time to tracer equilibrium in the global ocean. Ocean Science: An Interactive Open Access Journal of the European Geosciences Union, 5(1), 2009.

S. Rahmstorf. Bifurcation of the Atlantic thermohaline circulation in response to changes in the hydrological cycle. Nature, 378:9, 1995.

C.B. Ramsey, R.A. Staff, C.L. Bryant, F. Brock, H. Kitagawa, J. van der Plicht, G. Schlolaut, M.H. Marshall, A. Brauer, H.F. Lamb, et al. A complete terrestrial radiocarbon record for 11.2 to $52.8 \mathrm{kyr}$ BP. Science, 338(6105):370-374, 2012. 
PJ Reimer, MGL Baillie, E. Bard, A. Bayliss, JW Beck, PG Blackwell, C.B. Ramsey, CE Buck, GS Burr, RL Edwards, et al. INTCAL09 AND MARINE09 RADIOCARBON AGE CALIBRATION CURVES, 0-50,000 YEARS CAL BP. RADIOCARBON, 51(4):1111-1150, 2009.

Stephany I Rubin and Robert M Key. Separating natural and bomb-produced radiocarbon in the ocean: The potential alkalinity method. Global Biogeochemical Cycles, 16(4):52-1, 2002.

C. Rühlemann, S. Mulitza, P.J. Muller, G. Wefer, and R. Zahn. Warming of the tropical Atlantic Ocean and slowdown of thermohaline circulation during the last deglaciation. Nature, 402(6761):511-514, 1999.

R.L. Rutberg and S.L. Peacock. High-latitude forcing of interior ocean $813 \mathrm{C}$. Paleoceanography, 21(2):PA2012, 2006.

George B Rybicki and William H Press. Interpolation, realization, and reconstruction of noisy, irregularly sampled data. The Astrophysical Journal, 398:169-176, 1992.

Peter M Saunders. Practical conversion of pressure to depth. Journal of Physical Oceanography, 11:573-578, 1981.

NJ Shackleton, J-C Duplessy, M Arnold, P Maurice, MA Hall, and J Cartlidge. Radiocarbon age of last glacial Pacific deep water. Nature, 335(6192):708-711, 1988.

N.J. Shackleton, M.A. Hall, and E. Vincent. Phase relationships between millennialscale events 64,000-24,000 years ago. Paleoceanography, 15(6):565-569, 2000.

J.D. Shakun, P.U. Clark, F. He, S.A. Marcott, A.C. Mix, Z. Liu, B. Otto-Bliesner, A. Schmittner, and E. Bard. Global warming preceded by increasing carbon dioxide concentrations during the last deglaciation. Nature, 484(7392):49-54, 2012.

C. Siberlin. Time-scales of passive tracers in the ocean with paleoapplications. $\mathrm{PhD}$ thesis, Massachusetts Institute of Technology, 2010.

C. Siberlin and C. Wunsch. Oceanic tracer and proxy time scales revisited. Climate of the Past Discussions, 6:1589-1628, 2010.

LC Skinner and NJ Shackleton. Rapid transient changes in northeast Atlantic deep water ventilation age across Termination I. Paleoceanography, 19(2):PA2005, 2004.

LC Skinner and NJ Shackleton. An Atlantic lead over Pacific deep-water change across Termination I: implications for the application of the marine isotope stage stratigaphy. Quat Sci Rev, 24:571-580, 2005. 
LC Skinner, NJ Shackleton, and H. Elderfield. Millennial-scale variability of deepwater temperature and $\delta 180 \mathrm{dw}$ indicating deep-water source variations in the Northeast Atlantic, 0-34 cal. ka BP. Geochemistry Geophysics Geosystems, 4(12): 1098, 2003.

LC Skinner, S. Fallon, C. Waelbroeck, E. Michel, and S. Barker. Ventilation of the deep Southern Ocean and deglacial CO2 rise. Science, 328(5982):1147, 2010.

B. Stenni, V. Masson-Delmotte, S. Johnsen, J. Jouzel, A. Longinelli, E. Monnin, R. Röthlisberger, and E. Selmo. An oceanic cold reversal during the last deglaciation. Science, 293(5537):2074-2077, 2001.

Henry Stommel. Determination of water mass properties of water pumped down from the Ekman layer to the geostrophic flow below. Proceedings of the National Academy of Sciences, 76(7):3051-3055, 1979.

M. Stuiver and T.F. Braziunas. Sun, ocean, climate and atmospheric 14CO2: an evaluation of causal and spectral relationships. The Holocene, 3(4):289-305, 1993.

M. Stuiver, P.M. Grootes, and T.F. Braziunas. The GISP2 $\delta 18 \mathrm{O}$ climate record of the past 16,500 years and the role of the sun, ocean, and volcanoes. Quaternary Research, 44(3):341-354, 1995.

A.L. Thomas, G.M. Henderson, and L.F. Robinson. Interpretation of the 231Pa230Th paleocirculation proxy: New water-column measurements from the southwest Indian Ocean. Earth and Planetary Science Letters, 241(3-4):493-504, 2006.

J. E. Tierney and K. J. Anchukaitis. Identifying coherent spatio-temporal modes tropical African hydrology during the last 1500 years. Climate Dynamics, in prep., 2011.

M Tomczak Jr. Bass Strait water intrusions in the Tasman Sea and mean temperaturesalinity curves. Marine and Freshwater Research, 32(5):699-708, 1981.

E. Tziperman and A. Hecht. Circulation in the eastern Levantine Basin determined by inverse methods. J. Phys. Oceanogr, 18(3):506-518, 1988.

L. Vidal, RR Schneider, O. Marchal, T. Bickert, TF Stocker, and G. Wefer. Link between the North and South Atlantic during the Heinrich events of the last glacial period. Climate Dynamics, 15(12):909-919, 1999.

C. Waelbroeck, C. Levi, JC Duplessy, L. Labeyrie, E. Michel, E. Cortijo, F. Bassinot, and F. Guichard. Distant origin of circulation changes in the Indian Ocean during the last deglaciation. Earth and Planetary Science Letters, 243(1-2):244-251, 2006. 
C. Waelbroeck, LC Skinner, L. Labeyrie, J.C. Duplessy, E. Michel, N.V. Riveiros, J.M. Gherardi, and F. Dewilde. The timing of deglacial circulation changes in the Atlantic. Paleoceanography, 26(3):PA3213, 2011.

C. Wunsch. Using transient tracers: the regularization problem. Tellus B, 39(5): 477-492, 1987.

C. Wunsch. Oceanic age and transient tracers: Analytical and numerical solutions. JOURNAL OF GEOPHYSICAL RESEARCH-ALL SERIES-, 107(6; SECT 3): $1-1,2002$.

C. Wunsch. Determining paleoceanographic circulations, with emphasis on the Last Glacial Maximum. Quaternary Science Reviews, 22(2):371-385, 2003.

C. Wunsch. Discrete inverse and state estimation problems: with geophysical fluid applications. Cambridge Univ Pr, 2006.

C. Wunsch and P. Heimbach. How long to oceanic tracer and proxy equilibrium? Quaternary Science Reviews, 27(7-8):637-651, 2008.

C. Wunsch, M. Whitfield, DJ Webb, WJ Jenkins, C. Wunsch, M. Whitfield, DJ Webb, and WJ Jenkins. Eclectic Modelling of the North Atlantic. II. Transient Tracers and the Ventilation of the Eastern Basin Thermocline [and Discussion]. Philosophical Transactions of the Royal Society of London. Series A, Mathematical and Physical Sciences, 325(1583):201-236, 1988.

Carl Wunsch and Patrick Heimbach. Two Decades of the Atlantic Meridional Overturning Circulation: Anatomy, Variations, Extremes, Prediction, and Overcoming Its Limitations. Journal of Climate, (2013), 2013.

R. Zahn and A. Stüber. Suborbital intermediate water variability inferred from paired benthic foraminiferal $\mathrm{Cd} / \mathrm{Ca}$ and $\delta^{13} \mathrm{C}$ in the tropical West Atlantic and linking with North Atlantic climates. Earth and Planetary Science Letters, 200(1-2):191$205,2002$.

M. Zarriess and A. Mackensen. The tropical rainbelt and productivity changes off northwest Africa: a 31,000-year high-resolution record. Marine Micropaleontology, 76(3-4):76-91, 2010.

M. Zarriess and A. Mackensen. Testing the impact of seasonal phytodetritus deposition on $\delta 13 \mathrm{C}$ of epibenthic foraminifer Cibicidoides wuellerstorfi: A 31,000 year high-resolution record from the northwest African continental slope. Paleoceanography, 26(2):PA2202, 2011. 